\title{
WestVirginiaUniversity
}

THE RESEARCH REPOSITORY @ WVU

Graduate Theses, Dissertations, and Problem Reports

2014

\section{Alfven Wave Propagation in Inhomogeneous Plasmas}

Stephanie Sears

Follow this and additional works at: https://researchrepository.wvu.edu/etd

\section{Recommended Citation}

Sears, Stephanie, "Alfven Wave Propagation in Inhomogeneous Plasmas" (2014). Graduate Theses,

Dissertations, and Problem Reports. 7127.

https://researchrepository.wvu.edu/etd/7127

This Dissertation is protected by copyright and/or related rights. It has been brought to you by the The Research Repository @ WVU with permission from the rights-holder(s). You are free to use this Dissertation in any way that is permitted by the copyright and related rights legislation that applies to your use. For other uses you must obtain permission from the rights-holder(s) directly, unless additional rights are indicated by a Creative Commons license in the record and/ or on the work itself. This Dissertation has been accepted for inclusion in WVU Graduate Theses, Dissertations, and Problem Reports collection by an authorized administrator of The Research Repository @ WVU.

For more information, please contact researchrepository@mail.wvu.edu. 


\title{
Alfvén Wave Propagation in Inhomogeneous Plasmas
}

\author{
Stephanie Sears
}

Dissertation submitted to the

Eberly College of Arts and Sciences

at West Virginia University

in partial fulfillment of the requirements

for the degree of

Doctor of Philosophy

in

Physics

Dr. Earl Scime, Ph.D., Chair

Dr. Paul Cassak, Ph.D.

Dr. H. Arthur Weldon, Ph.D.

Dr. Felix Julian Schulze, Ph.D.

Dr. Edgar Fuller, Ph.D.

Department of Physics and Astronomy

Morgantown, West Virginia

2014

Keywords: Plasma; Alfvén Waves; Helicon

Copyright 2014 Stephanie Sears 


\begin{abstract}
Damping of Alfvén waves is one of the most likely mechanisms for ion heating in the solar corona. Density gradients have significant but poorlyunderstood effects on energy transfer and Alfvén wave propagation in partially ionized plasmas, such as those found in the solar chromosphere. Reflection of Alfvén waves at density and magnetic field gradients can give rise to turbulence which sustains particle heating. ${ }^{[1],[2]}$ The density profile in the Hot hELIcon eXperiment (HELIX) varies strongly with radius, giving access to a wide range of Alfvén dynamics across the plasma column and providing an ideal environment to observe Alfvén wave-driven particle heating.

A new internal wave-launching antenna, situated at the edge of the highdensity core and the density-gradient region of HELIX has been used to excite low-frequency waves in argon plasma. The propagation behavior of the launched waves was measured with a small-scale (smaller than the ion gyroradius) magnetic sense coil at multiple radial locations across the plasma column (from the high-density core through the density gradient region). Time-resolved laser induced fluorescence (LIF) and Langmuir probe measurements also yield insight into the plasma response to the perturbation.

This dissertation presents cross-spectral and wavelet analysis of low-frequency waves in a helicon plasma with a strong density gradient. Building on the work of Houshmandyar, ${ }^{[3]}$ shear Alfvén waves were launched in a helicon plasma source with a strong density gradient. Alfvén wave turbulence is suggested from phase angle and wavelet analysis of magnetic sense coil probe measurements. The perturbation wavelength derived from phase angle measurements is consistent with predictions from the full Alfvén wave dispersion relation (taking electron Landua damping, electron-ion collisions, and finite frequency effects into account). Time-resolved LIF measurements across the plasma column suggest ion heating where the turbulence is strongest. Timeresolved Langmuir probe measurements show electron heating in response to the launched Alfvén waves.
\end{abstract}


This work is dedicated

to the spirit of Shakespeare's sister and every woman

who has ever worked

to build a room of her own. 


\section{ACKNOWLEDGEMENTS}

The work presented here is my own, accomplished through years of hardwork and dedication; but it was made possible by the contributions of many people during that time.

My advisor, Dr. Earl Scime, has provided support, mentorship, and sound advice as I have transitioned from a new graduate student to a plasma physicist. My committee members have tested my understanding of physics and the relationship between my research and the field as a whole; their scrutiny has improved my work and broadened my perspective. My coworkers and lab-mates have been invaluable in helping me advance my laboratory techniques and experimental research skills. They have also become my friends and respected colleagues through our time together. The office staff have made it possible for me to do my job and the folks in the machine shop have provided invaluable work and advice over the years.

My family members and dear friends have been with me through the whole journey. Without their care, support, comfort, and comradeship, I would not enjoy the successes I have achieved to this day.

All the professors, researchers, and collaborators I have had the privi-

lege to interact with during my time at WVU have given me a breadth of knowledge and experience that has directly improved my work and research. Through their willingness to teach and share, I have become a better student, a better scientist, and a better member of the scientific community.

Finally and most significantly, my dearest friend and number one fan, my partner Josh, has given me unconditional love and support throughout this undertaking. I love him with all my heart and look forward to the next phase of our adventures together. 


\section{Table of Contents}

List of Tables vii

List of Figures viii

List of Abbreviations and Symbols xii

1 Introduction 1

2 Alfvén Waves and Heating Ions 6

2.1 Alfvén Waves . . . . . . . . . . . . . . . . . 6 6

2.2 Alfvén Wave Heating of Ions . . . . . . . . . . . . . . . 17

3 Experimental Apparatus 20

3.1 Introduction to Helicon Plasmas . . . . . . . . . . . . . . . 20

3.2 The HELIX/LEIA System . . . . . . . . . . . . . . . . 24

3.3 Pulsed Experiment Timing . . . . . . . . . . . . . . 32

4 Magnetic Sense Coil Probes 34

4.1 Magnetic Sense Coil Probe Theory and Design . . . . . . . . . 34

4.2 Magnetic Sense Coils Used in These Experiments . . . . . . . 35

4.2.1 Commercial Inductor Coils . . . . . . . . . . . . . . . 35

4.2.2 Hand-Wound Inductor Coils . . . . . . . . . . . . 37

5 Wave Launching Antennas 42

5.1 External Wave-Launching Antenna Design . . . . . . . . . . . 42

5.2 Internal Wave-Launching Antenna Designs . . . . . . . . . . . 44

5.2.1 Hand-Wound Transverse Antenna . . . . . . . . . . . . 44

5.2.2 Conducting Mesh Grid Antenna . . . . . . . . . . 46

6 Magnetic Probe Measurements 50

6.1 Steady-State Density Measurements and Perpendicular Length

Scales . . . . . . . . . . . . . . . . 50

6.2 Phase Angle Measurements . . . . . . . . . . . . . . . . . . 51

6.3 Time Series Analysis Techniques . . . . . . . . . . . . . . . . . 59

6.3.1 The Fourier Transform . . . . . . . . . . . . . 62

6.3.2 The Wavelet Transform . . . . . . . . . . . . . 67

6.3.3 Wavelet Analysis of Magnetic Sense Coil Probe Signals 71 
7 Time-Resolved Laser-Induced Fluorescence (LIF) Measurements 82

7.1 Laser-Induced Fluorescence Background and Theory . . . . . . 82

7.2 Time-Resolved LIF Measurements . . . . . . . . . . . . . . . . 90

8 Time-Resolved Langmuir Probe Measurements 122

8.1 Langmuir Probe Theory . . . . . . . . . . . . . . . . . . . . . 122

8.2 Time-Resolved Langmuir Probe Measurements . . . . . . . . . 131

9 Summary and Conclusions 143

$\begin{array}{ll}\text { Bibliography } & 146\end{array}$

Appendix A: MATLAB Code for Analyzing Time-Resolved Langmuir $\begin{array}{ll}\text { Probe Data } & 156\end{array}$

Appendix B: MATLAB Code for Wavelet Analysis 160 


\section{List of Tables}

1 Characteristic plasma parameters for HELIX. . . . . . . . . 31 


\section{List of Figures}

1.1 Illustration of Temperature vs. Altitude in the Solar Atmosphere 2

2.1 Cartoon Illustration of Shear and Compressional Alfvén Waves 7

2.2 Illustration of Counter-Propagating Alfvén Waves In the Solar Corona . . . . . . . . . . . . . . . . 19

3.1 Distinct Helicon Source Modes Depending on Magnetic Field and RF Power . . . . . . . . . . . . . . . . . . . 23

3.2 Schematic of the HELIX-LEIA System . . . . . . . . . . 25

3.3 Picture of the HELIX-LEIA System in Operation with Argon Plasma . . . . . . . . . . . . . . 26

3.4 Schematic of the HELIX Chamber With Diagnostics . . . . . 27

3.5 Diagram of the Impedance Matching Network for Coupling RF Power into HELIX. . . . . . . . . . . . . . . . . . . . . . 30

3.6 Schematic Drawing of the $m=+1$ Helical Antenna on HELIX. 30

4.1 Hand-Wound Magnetic Sense Coil Probe Used in This Work . 39

4.2 Magnetic Sense Coil Probe Time Series Plotted with Radial Location Across the Plasma Column . . . . . . . . . . . . . . 41

5.1 Schematic of HELIX With External Rectangular Transverse Wave-Launching Antenna . . . . . . . . . . . . . . . 43

5.2 Schematic Drawing of Internal Circular Transverse Wave-Launching Antenna . . . . . . . . . . . . . . . . . . 45

5.3 Internal Mesh Grid Wave-Launching Antenna . . . . . . . . . 46

5.4 Current Signal on the Perturbation Antenna For a Complete 60 ms Plasma Discharge . . . . . . . . . . . . . . . . . 48

5.5 Current Signal on the Perturbation Antenna For $1 \mathrm{~ms}$ During the Perturbation Pulse . . . . . . . . . . . . . . . . . 49

6.1 Steady-State Density and Gradient Density Scale Length Measurements . . . . . . . . . . . . . . 52

6.2 Phase Angle Measurements . . . . . . . . . . . . . . . . . . 55

6.3 Wavelength Measured in HELIX Based on Phase Angle Measurements Compared to Theoretical Predictions . . . . . . . . 57 
6.4 Wavelength Measured in HELIX based on Phase Angle Measurements Compared to Predictions From the Full Alfvén Wave Dispersion Relation . . . . . . . . . . . . . . . . . . . 58

6.5 Illustration of a Wavelet Function (a Daubechies 4 Wavelet) with a Sine Wave for Comparison . . . . . . . . . . . . . . . . 69

6.6 Illustration of a Wavelet Function Undergoing Translation and Dilation .................. . . 70

6.7 Wavelet Analysis of Magnetic Sense Coil Probe Measurements at $r=0 \mathrm{~cm}$. . . . . . . . . . . . . . . . . . . . . . . . . . 75

6.8 Wavelet Analysis of Magnetic Sense Coil Probe Measurements at $r=0.25 \mathrm{~cm} \ldots \ldots \ldots \ldots$. . . . . . . . . . . 76

6.9 Wavelet Analysis of Magnetic Sense Coil Probe Measurements at $r=0.50 \mathrm{~cm}$. . . . . . . . . . . . . . . . . . . 77

6.10 Wavelet Analysis of Magnetic Sense Coil Probe Measurements at $r=0.75 \mathrm{~cm} \ldots \ldots \ldots \ldots$. . . . . . . . . . . . . 78

6.11 Wavelet Analysis of Magnetic Sense Coil Probe Measurements at $r=1.0 \mathrm{~cm}$. . . . . . . . . . . . . . . . . . . . . . . 79

6.12 Wavelet Analysis of Magnetic Sense Coil Probe Measurements at $r=1.25 \mathrm{~cm}$. . . . . . . . . . . . . . . . . . 80

6.13 Integrated Wave Power vs. Frequency Across the Plasma Column ...................... . . 81

7.1 Non-Resonant LIF Scheme for Argon II Metastable Ions . . . 83

7.2 Typical Example of an LIF Trace in Argon Plasma in HELIX 85

7.3 Zeeman Splitting for Argon II ions . . . . . . . . . . . . . . . 87

7.4 Example of a Typical Time-Resolved LIF Ion Velocity Distribution Function . . . . . . . . . . . . . . . . . . . . 92

7.5 Time-Resolved Ion Temperature at $r=0 \mathrm{~cm}$. . . . . . . . . . 94

7.6 Normalized Difference for the Ion Temperature Measurement at $r=0 \mathrm{~cm}$. . . . . . . . . . . . . . . . . . . . . . . . . 95

7.7 Time-Resolved Ion Temperature at $r=0.25 \mathrm{~cm} \ldots . . . . .996$

7.8 Normalized Difference for the Ion Temperature Measurement at $r=0.25 \mathrm{~cm}$. . . . . . . . . . . . . . . . . . . . . . . . . . . 97

7.9 Time-Resolved Ion Temperature at $r=0.50 \mathrm{~cm}$. . . . . . . . 98

7.10 Normalized Difference for the Ion Temperature Measurement at $r=0.50 \mathrm{~cm} \ldots \ldots \ldots$. . . . . . . . . . . . . . . . . . 99

7.11 Time-Resolved Ion Temperature at $r=0.50 \mathrm{~cm}$, Second Measurement . . . . . . . . . . . . . . . . 100 
7.12 Normalized Difference for the Ion Temperature Measurement at $r=0.50 \mathrm{~cm}$, Second Measurement . . . . . . . . . . . . 101

7.13 Time-Resolved Ion Temperature at $r=0.75 \mathrm{~cm}$. . . . . . . . . 102

7.14 Normalized Difference for the Ion Temperature Measurement at $r=0.75 \mathrm{~cm} \ldots \ldots \ldots$. . . . . . . . . . . . 103

7.15 Time-Resolved Ion Temperature at $r=1 \mathrm{~cm} \ldots$. . . . . . . . 104

7.16 Normalized Difference for the Ion Temperature Measurement at $r=1 \mathrm{~cm}$. . . . . . . . . . . . . . . . . . . . . . . 105

7.17 Normalized Difference in Ion Temperature Across the Plasma Column .................... . 106

7.18 Time-Resolved LIF Signal Amplitude at $r=0 \mathrm{~cm}$. . . . . . . . 108

7.19 Normalized Difference for the LIF Amplitude Measurement at

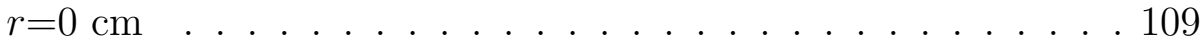

7.20 Time-Resolved LIF Signal Amplitude at $r=0.25 \mathrm{~cm}$. . . . . 110

7.21 Normalized Difference for the LIF Amplitude Measurement at $r=0.25 \mathrm{~cm} \ldots \ldots \ldots \ldots . \ldots . \ldots . \ldots 111$

7.22 Time-Resolved LIF Signal Amplitude at $r=0.50 \mathrm{~cm}$. . . . . . 112

7.23 Normalized Difference for the LIF Amplitude Measurement at $r=0.50 \mathrm{~cm}$. . . . . . . . . . . . . . . . . . 113

7.24 Time-Resolved LIF Signal Amplitude at $r=0.50 \mathrm{~cm}$, Second Measurement . . . . . . . . . . . . . . . . . . 114

7.25 Normalized Difference for the LIF Amplitude Measurement at $r=0.50 \mathrm{~cm}$, Second Measurement . . . . . . . . . . . . 115

7.26 Time-Resolved LIF Signal Amplitude at $r=0.75 \mathrm{~cm}$. . . . . . 116

7.27 Normalized Difference for the LIF Amplitude Measurement at $r=0.75 \mathrm{~cm}$. . . . . . . . . . . . . . . . 117

7.28 Time-Resolved LIF Signal Amplitude at $r=1.0 \mathrm{~cm}$. . . . . . . 118

7.29 Normalized Difference for the LIF Amplitude Measurement at $r=1 \mathrm{~cm} \ldots \ldots \ldots \ldots \ldots \ldots . \ldots \ldots 119 . \ldots \ldots$

7.30 Normalized Difference in LIF Signal Amplitude Across the Plasma Column . . . . . . . . . . . . . . . . . . . . . 121

8.1 Idealized Langmuir Probe I-V curve . . . . . . . . . . . . . . . 123

8.2 Schematic Drawing of Langmuir Probe with RF Compensation Used in This Work . . . . . . . . . . . . . . . . . . . . . . 130

8.3 Electron Density vs. Time Across the Plasma Column, Passing Through the Driven Current Channel . . . . . . . . . . . 135 
8.4 Electron Temperature vs. Time Across the Plasma Column, Passing Through the Driven Current Channel . . . . . . . . . 136

8.5 Electron Temperature Change Normalized to the Steady State Electron Temperature, Passing Through the Driven Current Channel . . . . . . . . . . . . . . . . . . . 137

8.6 Electron Density vs. Time Across the Plasma Column, Isolated from the Driven Current Channel . . . . . . . . . . . . 138

8.7 Electron Temperature vs. Time Across the Plasma Column, Isolated from the Driven Current Channel . . . . . . . . . . . 139

8.8 Electron Temperature Change Normalized to the Steady State Electron Temperature, Isolated from the Driven Current Channel . . . . . . . . . . . . . . . . . . . . . . . 140

8.9 Relative Change in Electron Temperature vs. Radius . . . . 141

8.10 Electron Temperature Heating Observations in LAPD . . . . . 142 


\title{
List of Abbreviations and Symbols
}

\author{
$\vec{A} \quad$ Vector Potential \\ $\beta \quad$ Ratio of Thermal Pressure to Magnetic Pressure \\ $\vec{B} \quad$ Total Magnetic Field \\ $\vec{B}_{0} \quad$ Equilibrium Magnetic Field \\ $\vec{B}_{1} \quad$ Perturbed Magnetic Field \\ $B_{r} \quad$ Radial Component of Magnetic Field \\ $B_{\theta} \quad$ Azimuthal Component of Magnetic Field \\ $B_{z} \quad$ Axial Component of Magnetic Field \\ $c_{s} \quad$ Ion Sound Speed \\ $\delta_{e} \quad$ Electron Skin Depth \\ $\epsilon_{m} \quad$ Electron/Ion mass ratio \\ $\vec{E} \quad$ Total Electric Field \\ $E_{r} \quad$ Radial Component of Magnetic Field \\ $E_{\theta} \quad$ Azimuthal Component of Magnetic Field \\ $E_{z} \quad$ Axial Component of Magnetic Field \\ $f \quad$ Total Particle Distribution Function \\ $f_{0} \quad$ Equilibrium Particle Distribution Function \\ $f_{1} \quad$ Perturbed Particle Distribution Function \\ $\vec{J} \quad$ Current Density \\ $k$ Total Wave Vector \\ $k_{\|} \quad$ Parallel Wave Number \\ $k_{\perp} \quad$ Perpendicular Wave Number \\ $\lambda_{d} \quad$ Debye shielding length \\ $\lambda_{e} \quad$ Electron inertial length (collisionless electron skin depth) \\ $\lambda_{i} \quad$ Ion inertial length (collisionless ion skin depth) \\ $\lambda_{\perp} \quad$ Parallel Wavelength \\ $\lambda_{\|} \quad$ Perpendicular Wavelength \\ $\lambda_{A}$ Alfvén Wavelength \\ $n$ Density \\ $\phi \quad$ Electric Potential \\ $\omega \quad$ Angular Frequency \\ $\omega_{c e}$ Electron Cyclotron Frequency \\ $\omega_{c i} \quad$ Ion Cyclotron Frequency \\ $\omega_{p e}$ Electron Plasma Frequency \\ $\omega_{p i} \quad$ Ion Plasma Frequency \\ $P \quad$ Isotropic Thermal Pressure \\ $\rho_{m}$ Total Mass Density
}




\title{
List of Abbreviations and Symbols (Continued)
}

\author{
$\rho_{n} \quad$ Neutral Mass Density \\ $\rho_{s} \quad$ Ion Sound Gyroradius \\ $\rho_{e} \quad$ Electron Gyroradius (Cyclotron Radius) \\ $\rho_{i} \quad$ Ion Gyroradius (Cyclotron Radius) \\ $\sigma \quad$ Conductivity \\ $T_{e} \quad$ Electron Temperature \\ $T_{i} \quad$ Ion Temperature \\ $T_{\|} \quad$ Parallel Temperature \\ $T_{\perp} \quad$ Perpendicular Temperature \\ $\vec{v} \quad$ Plasma Velocity \\ $v_{A} \quad$ Alfvén Speed \\ $v_{T e} \quad$ Electron Thermal Speed \\ $v_{T i} \quad$ Ion Thermal Speed
}




\section{Chapter 1}

\section{Introduction}

Alfvén wave dissipation is the primary physical mechanism that underlies one of the leading models of coronal ion heating in the solar atmosphere. In these models, the propagation and dissipation of, as well as interaction between, Alfvén waves are responsible for the temperature increase between the solar chromosphere and corona (see Figure 1.1). Erdélyi and Fedun have proposed low frequency wave dissipation as the source of coronal ion heating. ${ }^{[4]}$ More complex heating models propose high frequency waves which dissipate through cyclotron damping as the heating mechanism. Matthaeus et al. ${ }^{[1]}$ proposed resonant ion heating through high frequency waves generated by counter-propagating low frequency Alfvén waves while Dmitruk et al..$^{[5],[2]}$ proposed turbulent cascades as the source of high frequency waves that heat coronal ions. High-frequency wave generation and dissipation models are consistent with the anisotropic ion distributions $\left(T_{\perp} \gg T_{\|}\right)$observed in the solar corona ${ }^{[6],[7]}$ although the most likely result of turbulent cascades are waves that propagate perpendicular to the background magnetic field and 


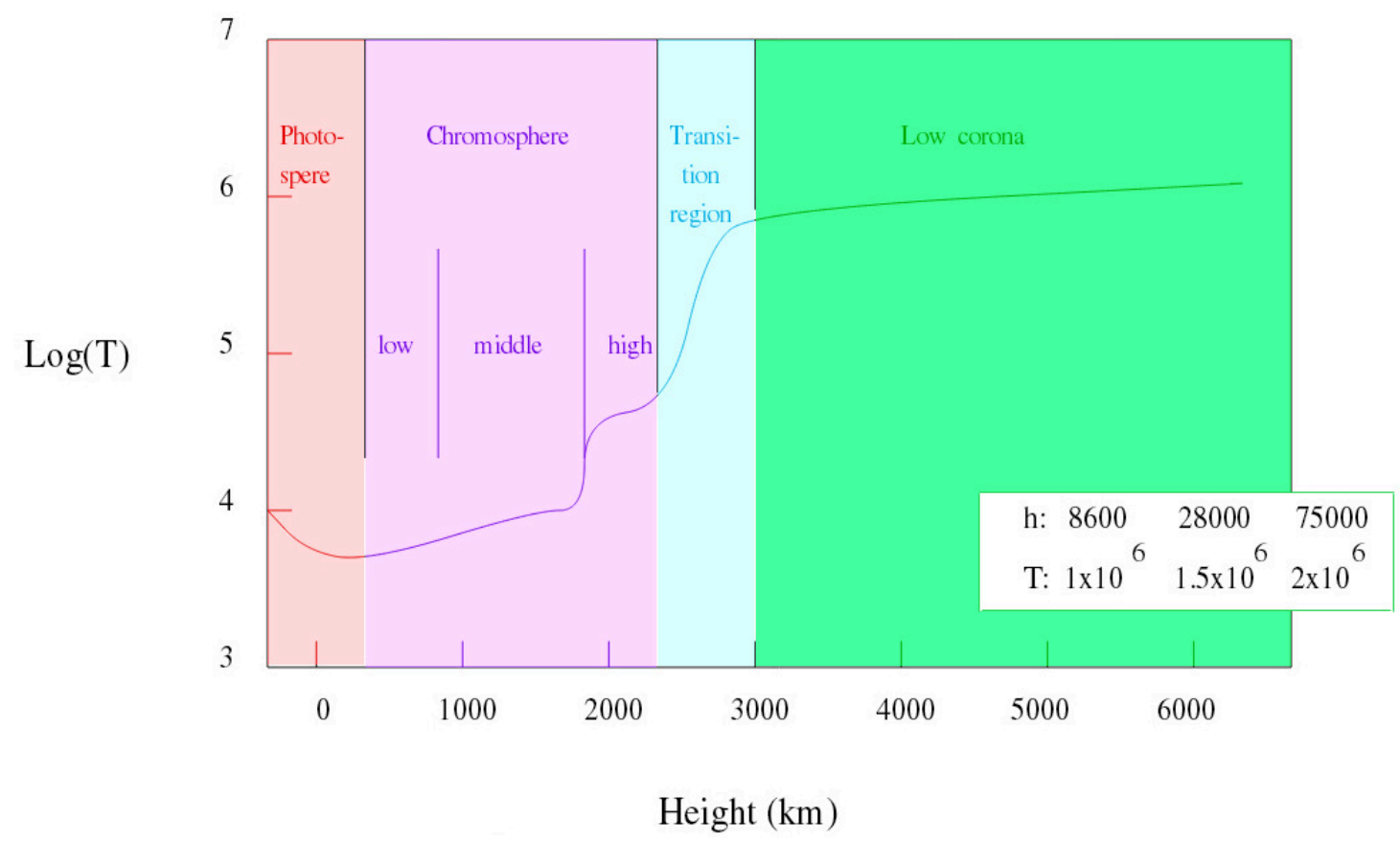

Figure 1.1: Observed temperature variation with altitude in the solar atmosphere. The inset at the right shows additional (increasing) temperature values for altitudes not included in the plot. Figure adapted from Erdélyi and Ballai. ${ }^{[11]}$

heat electrons instead of ions. ${ }^{[8]}$ Additional physical processes that convert perpendicular-propagating waves to parallel-propagating waves must be included in order to generate ion heating in these turbulent models. ${ }^{[5],[2],[9]}$ Chandran ${ }^{[10]}$ proposed a non-resonant coronal heating model which employs weak-turbulence analysis and the transfer of energy to high frequency fast waves and Alfvén waves which heat ions at a magnetic field gradient.

Because the wavelength of Alfvén waves is on the order of tens of meters 
in a typical laboratory plasma, investigation of Alfvén wave propagation in a laboratory environment requires plasma sources on the order of tens of meters in length, or high density sources in which the Alfvén wavelength is shorter. Helicon sources, with central densities on the order of $10^{13} \mathrm{~cm}^{-3}$ and Alfvén wavelengths on the order of a few meters, are ideal for studying Alfvén wave dynamics in a compact system. This dissertation reports experimental observations of shear Alfvén waves and the resulting plasma response across the strong density gradient region in the center of a helicon plasma source.

Extensive work on Alfvén wave propagation has been performed in the LArge Plasma Device (LAPD) facility at UCLA. ${ }^{[12]}$ LAPD investigations into Alfvén wave topics as diverse as the propagation of Alfvén waves in expanding plasmas; ${ }^{[13]}$ the behavior of Alfvén waves near the ion cyclotron frequency; ${ }^{[14]}$ the motion of ions in response to a shear Alfvén wave; ${ }^{[15]}$ and shear Alfvén wave propagation from the kinetic to the inertial regime ${ }^{[16]}$ have all been reported. The plasma density in the LAPD system is on the order of $10^{12} \mathrm{~cm}^{-3}$, which yields an Alfvén wavelength on the order of $10 \mathrm{~m}$. LAPD is approximately $17 \mathrm{~m}$ in length, which makes laboratory investigation of Alfvén waves possible in a plasma of that density.

The propagation of shear Alfvén waves in a helicon plasma was demon- 
strated by Watts and Hanna in 2001. ${ }^{[17]}$ With the use of small, commercially available inductors, Watts and Hanna successfully launched and detected shear Alfvén waves in the Auburn Linear Experiment for Space Plasma Investigations (ALESPI). Watts and Hanna showed in subsequent work that the dispersion relation of the shear Alfvén waves could be changed significantly by adjusting the neutral fraction of the plasma. ${ }^{[18]}$ We note that in subsequent experiments, Watts was unable to reproduce those initial experimental results and therefore no additional studies were ever reported. ${ }^{[19]}$

Initial Alfvén wave studies in the HELIX-LEIA helicon source system were performed by Houshmandyar. ${ }^{[3]}$ To take advantage of the decrease in Alfvén wavelength, and the increase in ion cyclotron frequency, that occurs with lower mass ion species, that initial work was performed in helium. The Houshmandyar work focused on exploring the optimal magnetic field and pressure profile for the propagation of Alfvén waves in a helium plasma in HELIX. This work uses a driven shear wave, rather than a mode-converted driven compressional wave, a smaller magnetic sense coil probe with better spatial resolution, time-resolved LIF and time-resolved Langmuir probe measurements to study the wave propagation and plasma interaction in realtime in an argon plasma. This work also uses newly developed time-frequency 
analysis techniques to glean more information from the magnetic sense coil probe time series measurements. 


\section{Chapter 2}

\section{Alfvén Waves and Heating Ions}

\subsection{Alfvén Waves}

The concept of low-frequency $\left(\omega \ll \omega_{c i}\right)$ electromagnetic waves traveling through a perfect conductor with parallel phase speed $v_{a}=B_{0} / \sqrt{\mu_{0} m_{i} n_{i}}$ (where $B_{0}$ is the background magnetic field, $m_{i}$ is the ion mass, and $n_{i}$ is the ion density) was first proposed by Hannes Alfvén in 1942. ${ }^{[20]}$ These lowfrequency oscillations propagating in a magnetized fluid are now typically referred to as Alfvén waves in recognition of his work. Two distinct types of Alfvén modes exist, which are called by different names in different texts. One mode, called the fast mode, the compressional mode, or the magnetosonic mode, involves the compression of magnetic field lines and resembles a sound wave; this mode is characterized by magnetic field perturbations parallel to the background field as well as perpendicular to it. The other mode, called the shear mode, the torsional mode, or the Alfvén mode, involves shearing or twisting of magnetic field lines; this mode is characterized 


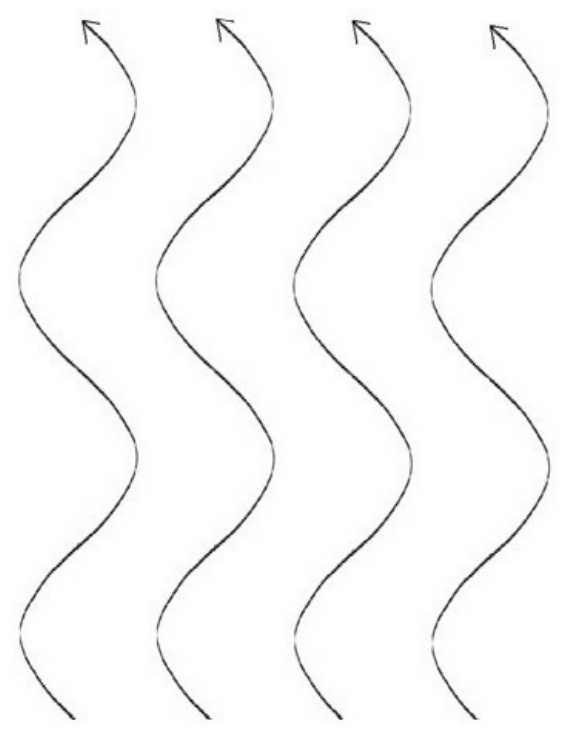

Shear Alfvén Wave

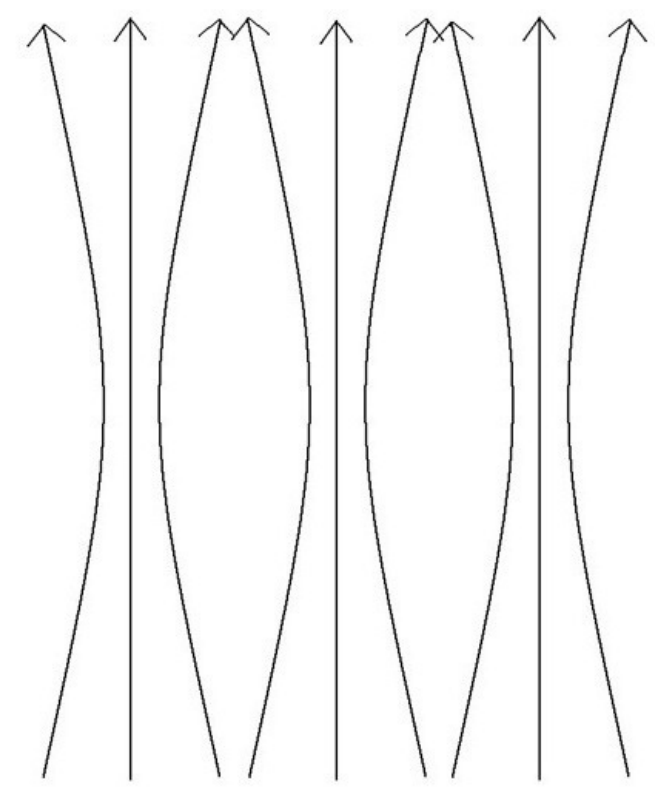

Compressional Alfvén Wave

Figure 2.1: Illustration of the perturbed magnetic field (in both cases $B_{0}$ is toward the top of the page) for shear and compressional Alfvén waves.

by magnetic field perturbations in the perpendicular direction only.

The shear mode has two $\beta$-dependent sub-types (where $\beta=\left(n K_{B} T\right) /\left(B^{2} / 2 \mu_{0}\right)$ is the ratio of thermal pressure to magnetic pressure). When the wavelength perpendicular to the background magnetic field, $\lambda_{\perp}$, is on the order of the electron inertial length $\left(\delta_{e}=c / \omega_{p e}\right)$ and $\beta<m_{e} / m_{i}$, magnetic effects dominate and two-fluid theory is necessary to accurately model the wave behavior; this is the "inertial" limit. When $\lambda_{\perp}$ is on the order of the ion sound gyrora- 
$\operatorname{dius}\left(\rho_{s}=c_{s} / \omega_{c i}=\sqrt{T_{e \|} / m_{i}} / \omega_{c i}\right)$ and $\beta>m_{e} / m_{i}$, thermal effects dominate; this is the "kinetic" limit. In these non-MHD regimes, parallel electric fields become significant and the waves are dispersive; that is, the wave behavior depends on perpendicular wavelength in addition to the parallel wavelength.

The Alfvén wave dispersion relation can be derived by starting with the ideal single fluid magnetohydrodynamic (MHD) equations in the cold, incompressible limit,

$$
\begin{gathered}
\nabla \times \vec{B}=\mu_{0} \vec{J} \\
\nabla \times \vec{E}=-\frac{\partial \vec{B}}{\partial t} \\
\vec{J}=\sigma(\vec{E}+\vec{v} \times \vec{B}) \\
\rho_{m} \frac{\mathrm{d} \vec{v}}{\mathrm{~d} t}=\vec{J} \times \vec{B}-\nabla P
\end{gathered}
$$

where $\mathrm{d} / \mathrm{d} t$ is the convective derivative $\partial / \partial t+\vec{v} \cdot \nabla, \rho_{m}$ is the plasma mass 
density, $\sigma$ is the plasma conductivity, $P$ is the isotropic thermal pressure, $\vec{J}$ is the current density, and $\vec{E}$ and $\vec{B}$ are the electric and magnetic fields. For low frequency waves, the displacement current in Equation 2.1 is negligible and can be ignored. The mass density and velocity are defined as

$$
\begin{aligned}
& \rho_{m}=\sum_{s} n_{s} m_{s} \\
& \vec{v}=\frac{\sum_{s} n_{s} m_{s} \vec{v}_{s}}{\rho_{m}}
\end{aligned}
$$

where the summation is over all species, denoted by the subscript $s$. Taking the zero-pressure regime limit leads to the shear Alfvén wave dispersion relation. ${ }^{[21]}$ In this limit the plasma is perfectly conducting $(\sigma \rightarrow \infty)$, which reduces Ohm's Law (Eqn. 2.3) to

$$
\vec{E}+\vec{v} \times \vec{B}=0
$$

The time dependent electric field perpendicular to the steady-state magnetic 
field gives rise to a polarization drift,

$$
\vec{v}_{s, \text { Polarization }}=\frac{m_{s}}{q_{s} B^{2}} \frac{d \vec{E}_{\perp}}{\mathrm{d} t}
$$

which generates a polarization current

$$
\begin{aligned}
\vec{J}_{\perp} & =\sum n_{s} q_{s} \vec{v}_{s} \\
& =\frac{\rho_{m}}{B^{2}} \frac{\mathrm{d} \vec{E}_{\perp}}{\mathrm{d} t}
\end{aligned}
$$

which can be rewritten as

$$
\begin{aligned}
\frac{d \overrightarrow{E_{\perp}}}{\mathrm{d} t} & =\frac{B^{2}}{\mu_{0} \rho_{m}} \mu_{0} \vec{J}_{\perp} \\
& =v_{A}^{2}\left(\nabla \times \vec{B}_{1}\right)_{\perp}
\end{aligned}
$$

where

$$
v_{A}^{2}=\sqrt{\frac{B^{2}}{\mu_{0} \rho_{m}}}
$$

is the Alfvén velocity. Combining the linearized forms of Equations 2.2 
and 2.10 yields the governing equations of these Alfvén modes

$$
\begin{array}{r}
\frac{\partial \vec{B}_{1}}{\partial t}=-\nabla \times \vec{E}_{1} \\
\frac{\partial \vec{E}_{\perp 1}}{\partial t}=v_{A}^{2}\left(\nabla \times \vec{B}_{1}\right)_{\perp} .
\end{array}
$$

Taking the $\hat{z}$ direction to be the direction of the background magnetic field, noting that $E_{z 1}$ is zero, and rewriting the curl operator as $\nabla=\nabla_{\perp}+\hat{z} \frac{\partial}{\partial z}$ gives the basic pair of coupled equations for MHD waves:

$$
\begin{array}{r}
\frac{\partial \vec{B}_{1}}{\partial t}=-\nabla_{\perp} \times \vec{E}_{\perp 1}-\hat{z} \times \frac{\partial \vec{E}_{\perp 1}}{\partial z} \\
\frac{\partial \vec{E}_{\perp 1}}{\partial t}=v_{a}^{2}\left(\nabla_{\perp} B_{z 1} \times \hat{z}+\hat{z} \times \frac{\partial \vec{B}_{\perp 1}}{\partial z}\right) .
\end{array}
$$

In the shear mode, where $B_{z 1}=0$, these equations become

$$
\begin{gathered}
\frac{\partial \vec{B}_{\perp 1}}{\partial t}=\frac{\partial}{\partial z}\left(\hat{z} \times \vec{E}_{\perp 1}\right) \\
\frac{\partial}{\partial t}\left(\hat{z} \times \vec{E}_{\perp 1}\right)=v_{a}^{2} \frac{\partial \vec{B}_{\perp 1}}{\partial z} .
\end{gathered}
$$

After taking the second time derivative of Equation 2.14a and substituting for $\partial / \partial t(\hat{z}) \times \vec{E}_{\perp 1}$ using Equation 2.14b, we get the wave equation for the 
shear mode,

$$
\frac{\partial^{2} \vec{B}_{\perp 1}}{\partial t^{2}}=v_{A}^{2} \frac{\partial^{2} \vec{B}_{\perp 1}}{\partial z^{2}}
$$

yielding the dispersion relation

$$
\omega^{2}=k_{\|}^{2} v_{A}^{2}
$$

which is the dispersion relation originally derived by Hannes Alfvén. ${ }^{[20]}$ When the perpendicular wavelength becomes small, a parallel component of the electic field arises and must be accounted for in the dispersion relation. When the perpendicular wavelength becomes comparable to the electron skin depth, $\delta_{e}=c / \omega_{p e}=c / \sqrt{4 \pi n_{e} e^{2} / m_{e}}$, the electron inertia becomes important and the dispersion relation must be modified to account for the perpendicular dynamics

$$
\omega=k \frac{B}{\sqrt{\mu_{0} \rho_{m}}} \frac{1}{\sqrt{\left(1+\left(k_{\perp} \delta_{e}\right)^{2}\right)}} .
$$

When the wave frequency becomes comparable to ion cyclotron frequency, this dispersion relation must again be modified to account for those finite 
frequency effects,

$$
\omega=k \frac{B}{\sqrt{\mu_{0} \rho_{m}}} \frac{\sqrt{1-\left(\omega / \omega_{c i}\right)^{2}}}{\sqrt{\left(1+\left(k_{\perp} \delta_{e}\right)^{2}\right)}} .
$$

Finally, using the relationship between wavelength and wavenumber, $k=$ $2 \pi / \lambda$, yields the modified inertial dispersion relation for the parallel Alfvén wavelength in terms of the driving frequency and the perpendicular wavenumber

$$
\lambda_{\|}=\frac{2 \pi B}{\omega \sqrt{\mu_{0} \rho_{m}}} \frac{\sqrt{1-\left(\omega / \omega_{c i}\right)^{2}}}{\sqrt{\left(1+\left(k_{\perp} \delta_{e}\right)^{2}\right)}} .
$$

When the the electron thermal speed exceeds the Alfvén speed, thermal effects become important, the perpendicular wavelength becomes comparable to the ion sound gyroradius, and the dispersion relation must also be modified to account for the perpendicular dyanmics for the kinetic Alfvén wave

$$
\lambda_{\|}=k \frac{2 \pi B}{\omega} \frac{1}{\sqrt{\mu_{0} \rho_{m}}} \sqrt{\left(1-\left(\omega / \omega_{c i}\right)^{2}+\left(k_{\perp} \rho_{s}\right)^{2}\right)} .
$$

In the compressional mode, where $B_{z 1} \neq 0$, the dispersion relation is obtained from taking the parallel $(\hat{z})$ component of Equation 2.13a and the 
cross product of Equation $2.13 \mathrm{~b}$ with $\hat{z}$ to get

$$
\begin{gathered}
\frac{\partial B_{z 1}}{\partial t}=-\hat{z} \cdot\left(\nabla_{\perp} \times \vec{E}_{\perp 1}\right)=-\nabla \cdot\left(\vec{E}_{\perp 1} \times \hat{z}\right) \\
\frac{\partial}{\partial t}\left(\vec{E}_{\perp 1} \times \hat{z}\right)=v_{A}^{2}\left(\nabla_{\perp} B_{z 1} \times \hat{z}+\hat{z} \times \frac{\partial \vec{B}_{\perp 1}}{\partial z}\right) \times \hat{z} \\
=v_{A}^{2}\left(-\nabla_{\perp} B_{z 1}+\frac{\partial \vec{B}_{\perp 1}}{\partial z}\right)
\end{gathered}
$$

Taking the time derivative of Equation 2.21a and substituting $\partial / \partial t\left(\vec{E}_{\perp 1} \times \hat{z}\right)$ from Equation 2.21b yields

$$
\begin{aligned}
\frac{\partial^{2} B_{z 1}}{\partial t^{2}} & =-v_{A}^{2} \cdot\left(-\nabla_{\perp} B_{z 1}+\frac{\partial \vec{B}_{\perp 1}}{\partial z}\right) \\
& =v_{A}^{2} \nabla_{\perp}^{2} B_{z 1}-v_{A}^{2} \frac{\partial}{\partial z}\left(\nabla \cdot \vec{B}_{\perp 1}\right)
\end{aligned}
$$

yielding the dispersion relation

$$
\omega^{2}=k^{2} v_{A}^{2}
$$

In cases where additional loss mechanisms, such as electron Landau damping and ion-neutral collisions, are present, a dispersion relation which incorporates these dynamics must be used. To account for these additional terms, the derivation found in Stix ${ }^{[22]}$ and Vincena et al. ${ }^{[23]}$ is useful. For an az- 
imuthally symmetric shear Alfvén wave propagating in an infinite, uniform plasma the general dispersion relation in Cartesian coordinates can be written as

$$
n_{\perp}^{2} n_{\|}^{2}=\left(n_{\|}^{2}-\epsilon_{x x}\right)\left(n_{\perp}^{2}-\epsilon_{\|}\right)+\epsilon_{x y}^{2}\left(n_{\perp}^{2}-\epsilon_{\|}\right) /\left(n^{2}-\epsilon_{x x}\right)
$$

where the dielectric tensor elements $\epsilon_{x x}$ and $\epsilon_{x y}$ are

$$
\epsilon_{x x}=\frac{1}{2}\left(\frac{\omega_{p i}}{\omega}\right)^{2}\left[\frac{\omega}{k_{\|} \bar{v}_{t}} Z\left(\frac{\omega-\omega_{c i}}{k_{\|} \bar{v}_{t}}\right)-\frac{\omega}{\omega+\omega_{c i}}\right]
$$

and

$$
\epsilon_{x y}=\frac{1}{2}\left(\frac{\omega_{p i}}{\omega}\right)^{2}\left[\frac{\omega}{k_{\|} \bar{v}_{t}} Z\left(\frac{\omega-\omega_{c i}}{k_{\|} \bar{v}_{t}}\right)+\frac{\omega}{\omega+\omega_{c i}}-2 \frac{\omega}{\omega_{c i}}\right]
$$

where $\bar{v}_{t}=2 T_{i} / m_{i}$ is the average ion thermal speed and $Z$ is the plasma dispersion function. ${ }^{[24]}$ The parallel dielectric tensor element if collisions can be ignored is given by

$$
\epsilon_{\|}=-\left(\frac{\omega_{p e}}{\omega}\right)^{2} \zeta_{e}^{2} Z^{\prime}\left(\zeta_{e}\right)
$$


where $\zeta_{e}=\omega / k_{\text {parallel }} \bar{v}_{e}$ is the ratio of the parallel wave speed to the average electron thermal speed and $Z^{\prime}$ is the derivative of the plasma dispersion function with respect to its argument. In order to account for electronion Coulomb interactions, this term is modified to include a Krook collision operator

$$
\epsilon_{\|}=-\left(\frac{\omega_{p e}}{\omega}\right)^{2} \zeta_{e} \eta_{e} Z^{\prime}\left(\eta_{e}\right)
$$

where the new argument of the plasma dispersion function is $\eta_{e}=\zeta_{e}(1+i \Gamma)$ and $\Gamma=\nu_{e i} / \omega$ is the ratio of the electron-ion collision frequency to the angular wave frequency. From Koch and Horton ${ }^{[25]}$ the electron-ion collision frequency is

$$
\nu_{e i}=2 \pi n e^{4} \ln \Lambda / m_{e}^{2} \bar{v}_{e}^{3},
$$

where $\ln \Lambda$ is the Coulomb logarithm. 


\subsection{Alfvén Wave Heating of Ions}

Alfvén wave dissipation is one of the leading explanations for ion heating in the solar corona (see Fig. 1.1). The existence of Alfvén waves in the solar corona has been suggested since the observation of Alfvén waves in the high-speed solar wind by Belcher in 1971. ${ }^{[26]}$ Recent observations from the Hinode spacecraft have provided strong evidence of Alfvén waves, ${ }^{[4]}$ including upward-propagating Alfvén waves in the chromosphere ${ }^{[27]}$ and mag-

netic reconnection sites ${ }^{[28],[29]}$ in the solar corona. While numerous models of Alfvén wave ion heating in the solar corona have been proposed and refined, these models fall into two basic categories: nonlinear damping of lowfrequency Alfvén waves ${ }^{[30],[31],[32]}$ and cyclotron damping of high-frequency Alfvén waves. ${ }^{[33],[34]}$

In 1999 Matthaeus et al. ${ }^{[1]}$ proposed that counter-propagating Alfvén waves in the solar corona were responsible for the observed particle heating at higher altitudes. According to this theory, large-scale motions in the chromosphere or photosphere generate Alfvén waves that travel upward along the magnetic field. At density and magnetic field gradients, some of these waves are reflected (see Figure 2.2). Interactions between the reflected waves and the original upward-propagating waves generate turbulence which 
transfers energy to smaller perpendicular length scales and provides efficient electron heating. Dmitruk et al. ${ }^{[2]}$ showed that these counter-propagating Alfvén waves were sufficient to sustain turbulence and heating consistent with observations of the solar corona. Matthaeus and Dmitruk argue that the turbulence responsible for heating electrons is also responsible for heating ions. In this work, we show that both electron and ion heating are observed in correlation with Alfvén wave turbulence in a helicon plasma. 


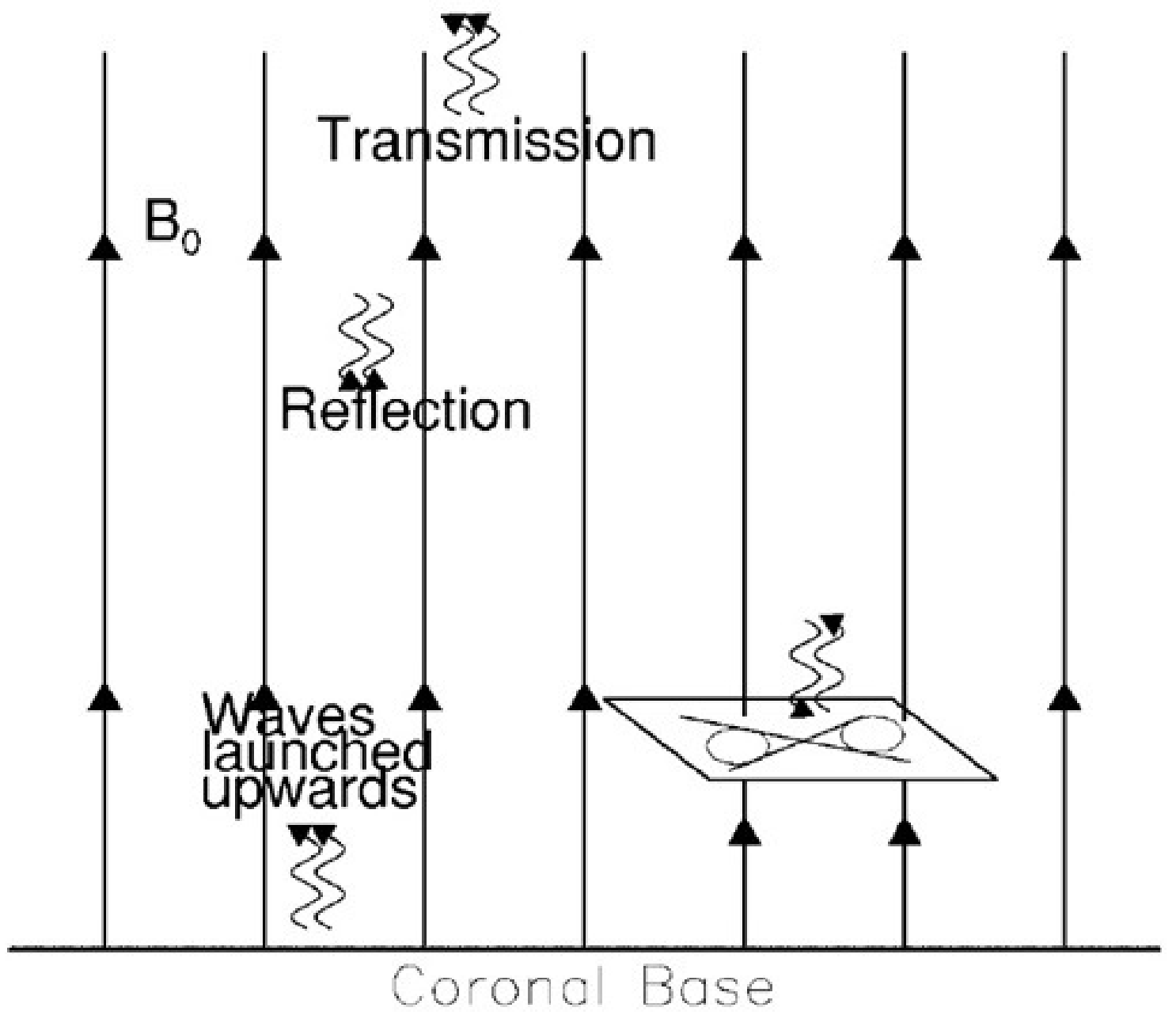

Figure 2.2: Illustration of Alfvén waves in the soloar corona showing reflection at density or magnetic field gradients. Interactions between counter-propagating Alfvén waves drive turbulence, which transfers energy to smaller perpendicular scales and leads to electron heating. These counterpropagating Alfvén waves have been shown to be sufficient to sustain turbulence and heating. ${ }^{[2]}$ Figure adapted from Matthaeus et al. ${ }^{[1]}$ 


\section{Chapter 3}

\section{Experimental Apparatus}

\subsection{Introduction to Helicon Plasmas}

Helicon plasma sources produce high density, current free plasma with typical electron densities in the $10^{19} \mathrm{~m}^{-3}$ range and typical electron temperatures from 5-10 eV. Helicon sources can be operated in both steadystate and pulsed modes. Helicon plasma sources have applications in basic plasma physics, ${ }^{[35],[36]}$ semi-conductor plasma processing, ${ }^{[37],[38]}$ space craft

propulsion, ${ }^{[39],[40],[41],[42]}$ plasma-surface interaction, laboratory studies of space plasma phenomena, ${ }^{[43][44]}$ and laboratory-based Alfvén wave studies. ${ }^{[45],[46],[47]}$

Helicon waves are right-hand circularly polarized, bounded electromagnetic waves with $\omega_{c i} \ll \omega \ll \omega_{c e}$, where $\omega_{c i}$ is the ion cyclotron frequency and $\omega_{c e}$ is the electron cyclotron frequency. These waves are called whistler waves ( $\mathrm{R}$ waves) in free (unbounded) cases due to their characteristic descending 
tones. The dispersion relation, ignoring collisions, is

$$
\frac{c^{2} k^{2}}{\omega^{2}}=1-\frac{\omega_{p e}^{2} / \omega^{2}}{1-\left(\omega_{c e} / \omega\right) \cos \theta}
$$

where $\omega_{p e}=\sqrt{n e^{2} / \epsilon_{0} m_{e}}$ is the electron plasma frequency, $\omega_{c e}=q B / m_{e}$ is the electron cyclotron frequency, and $\theta$ is the angle of propagation relative to the background magnetic field. In the $\omega \ll \omega_{c e} \ll \omega_{p e}$ regime, the dispersion relation becomes

$$
\frac{c^{2} k^{2}}{\omega^{2}}=\frac{\omega_{p e}^{2}}{\left(\omega_{c e} / \omega\right) \cos \theta} .
$$

Letting $k=k_{\text {total }}$ and $k_{\|}=k \cos \theta$, Eqn. 3.2 becomes

$$
k=\frac{\omega}{k_{\|}} \frac{\omega_{p e}^{2}}{\omega_{c e} c^{2}} .
$$

When the plasma and cyclotron frequences are substituted into Eqn. 3.3, the relation can be solved for the plasma density to yield

$$
n=\frac{k B}{\mu_{0} e}\left(\frac{k_{\|}}{\omega}\right)
$$


where $k_{\|}$is the parallel wavenumber, $k=\sqrt{k_{\perp}^{2}+k_{\|}^{2}}$ is the magnitude of the wavevector, $B$ is the magnitude of the background magnetic field, $\mu_{0}$ is the permeability of free space, $e$ is the elementary charge, and $\omega$ is the wave frequency. ${ }^{[48]}$

Helicon sources operate in three distinct modes, transitions between which are characterized by discontinuous changes in the plasma density (shown in Fig. 3.1). Which mode a helicon plasma source is in depends on both the RF power input and the magnetic field strength. ${ }^{[49]}$ The highest density (helicon) mode is only achievable if both the magnetic field strength and the RF power are above a certain threshold.

The capacitive mode ( $\mathrm{E}$ mode) and inductive mode ( $\mathrm{H}$ mode) are both low density modes (densities on the order of $10^{10} \mathrm{~cm}^{-3}$ and $10^{11} \mathrm{~cm}^{-3}$ ) characterized by limited penetration of deposited RF power. In capacitive mode the skin depth of the plasma is larger than the system itself and it is the capacitive voltage difference between the antenna and the plasma that accelerates free charges which sustain and heat the plasma. In inductive mode, the density is higher than capacitive mode, the skin depth is smaller than the size of the system, and RF electric fields induce currents in the plasma, which sustain and heat it. The helicon mode ( $\mathrm{W}$ mode) is a high density 


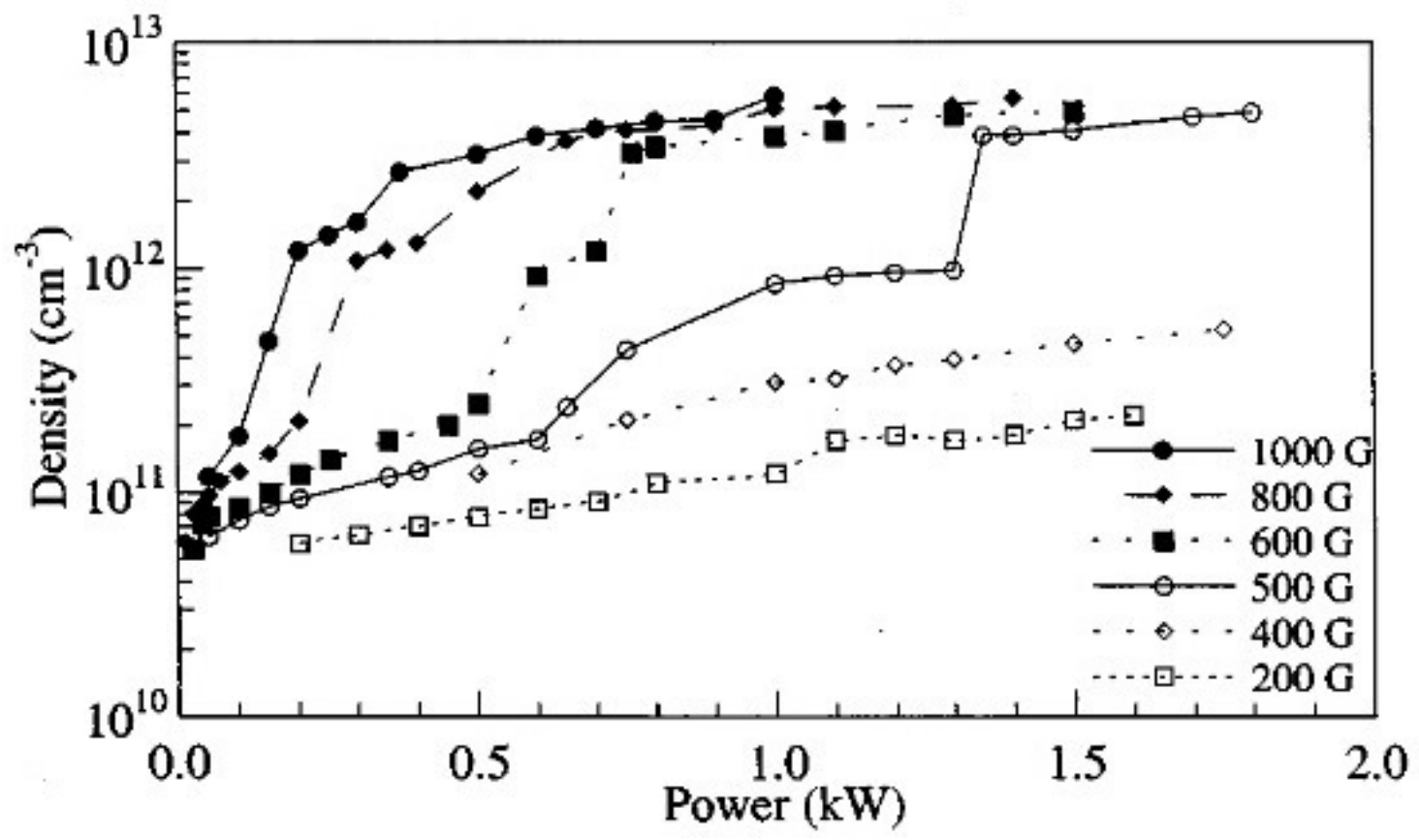

Figure 3.1: Electron density as a function of RF power for six different magnetic field strength values at a fill pressure of 6 mTorr. ${ }^{[49]}$ Note that for low field strengths (200 and $400 \mathrm{G}$ ), the helicon mode is never achieved, even at the highest values of RF power input. 
mode characterized by efficient RF power deposition and absorption of the $\mathrm{RF}$ wave sustains and heats the plasma.

While helicon plasma sources are ideal for industry applications and scientific applications, the mechanism responsible for RF power coupling into the plasma in helicon mode is poorly understood and continues to be investigated. ${ }^{[50]}$ Some proposed mechanisms for the efficiency of helicon source operation are collisional processes, ${ }^{[51],[52]}$ helicon wave penetration, ${ }^{[53]}$ antenna-localized acceleration, ${ }^{[54],[55]}$ Landau damping, ${ }^{[56],[57]}$ lower hybrid frequency mode conversion, ${ }^{[58]}$ and non-linear trapping of fast electrons. ${ }^{[59]}$ Factors such as neutral pressure, antenna design, and magnetic field strength all effect the RF power coupling and the ultimate density profile achievable in a helicon source plasma.

\subsection{The HELIX/LEIA System}

The WVU helicon plasma experiment consists of two distinct regions: (1) The Hot hELIcon eXperiment (HELIX) source region and (2) the Large Experiment on Instabilities and Anisotropies (LEIA) expansion region (See Fig. 3.2).

One end of HELIX is connected to a glass cross vacuum chamber which 


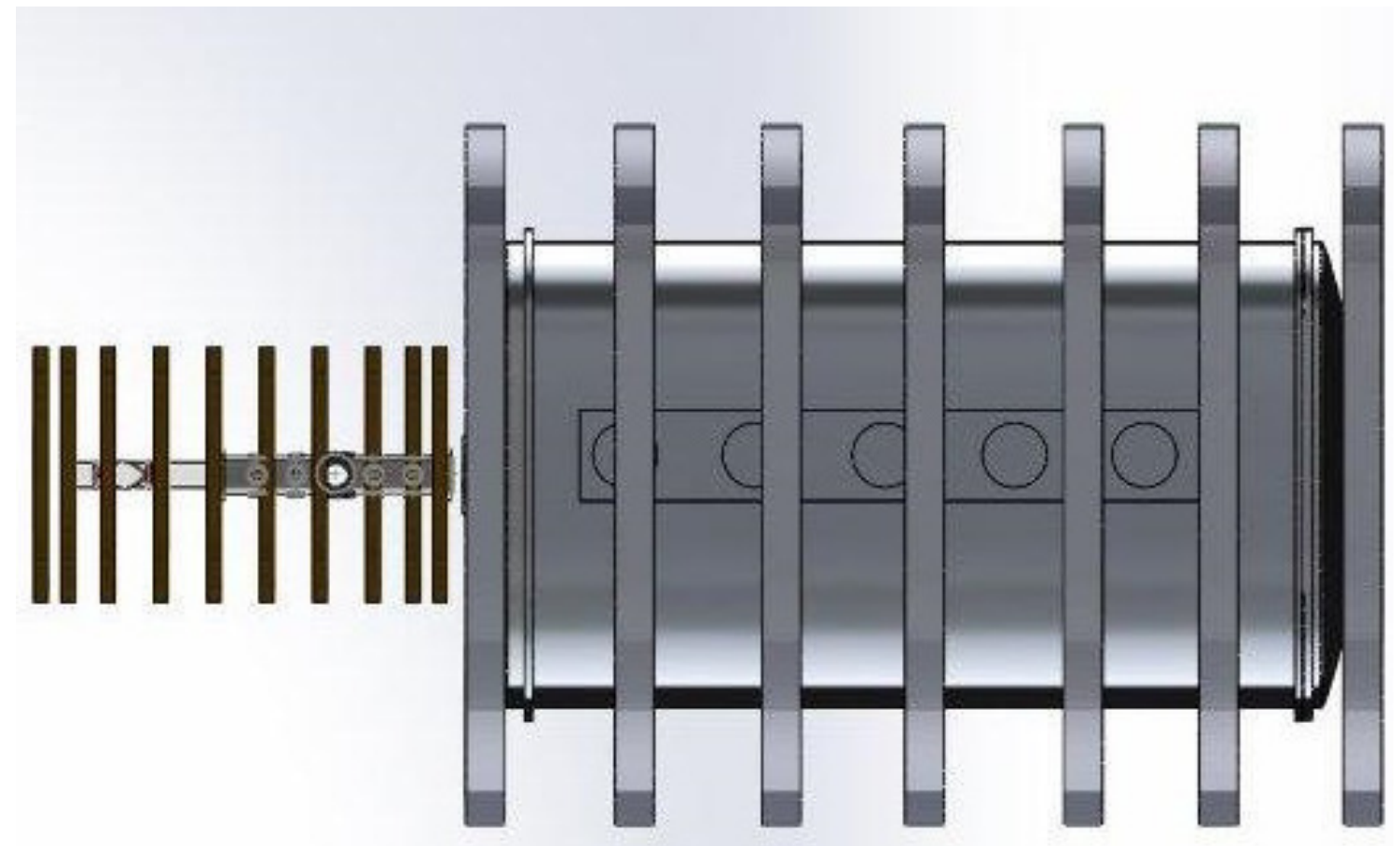

Figure 3.2: Schematic of the HELIX-LEIA chamber. Ten water-cooled electromagnets produce an almost uniform, steady-state axial magnetic field up to $1.2 \mathrm{kG}$ in HELIX. Seven water-cooled electromagnets produce a steadystate axial magnetic field up to $150 \mathrm{G}$ in LEIA. 


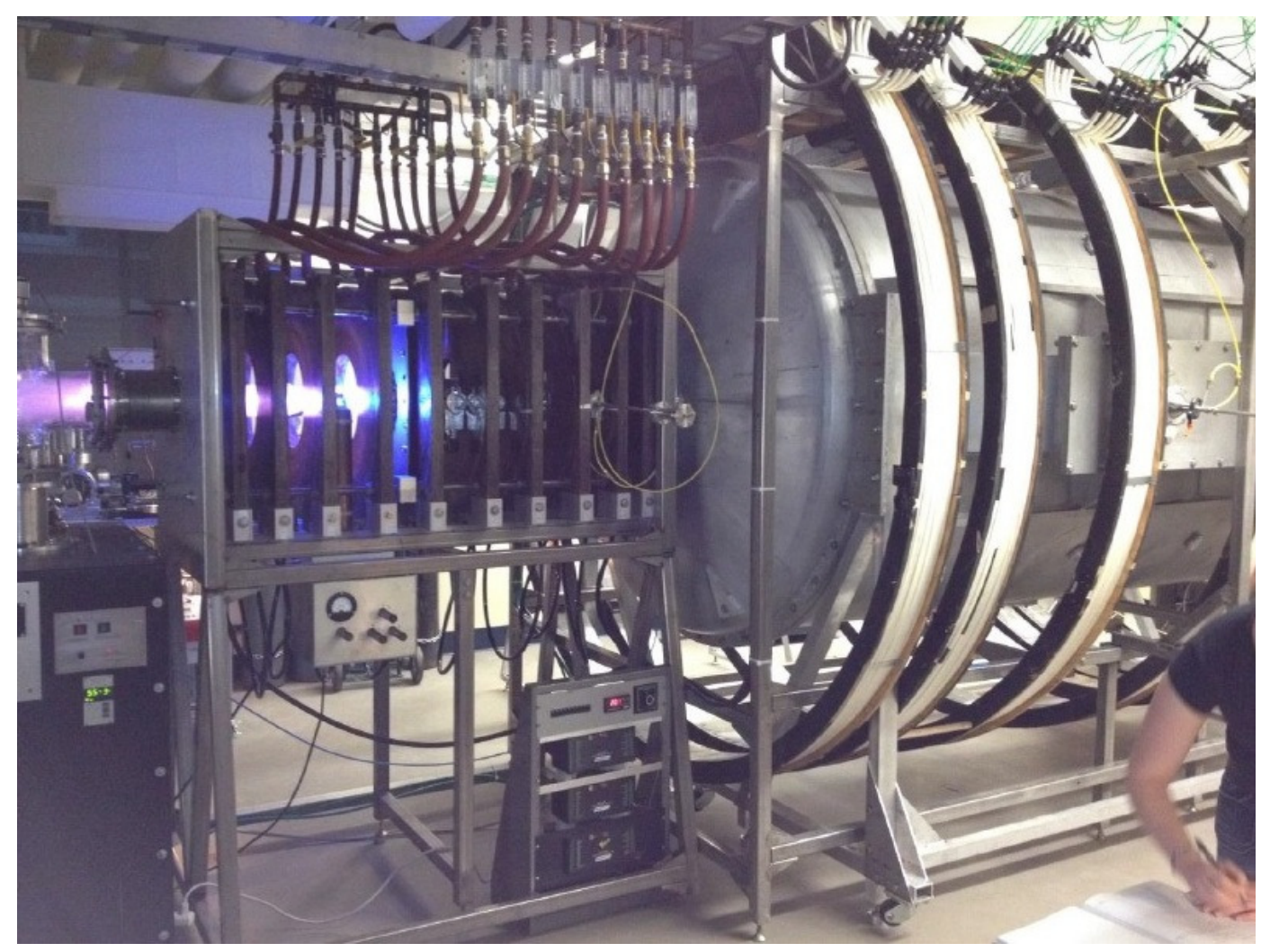

Figure 3.3: The HELIX-LEIA system in operation. To the far left is the glass cross chamber that connects to the turbomolecular pumping station. Just to the right of that is HELIX (with argon plasma) and further to the right the LEIA chamber and its $\sim 3$ meter diameter electromagnets can be seen. 


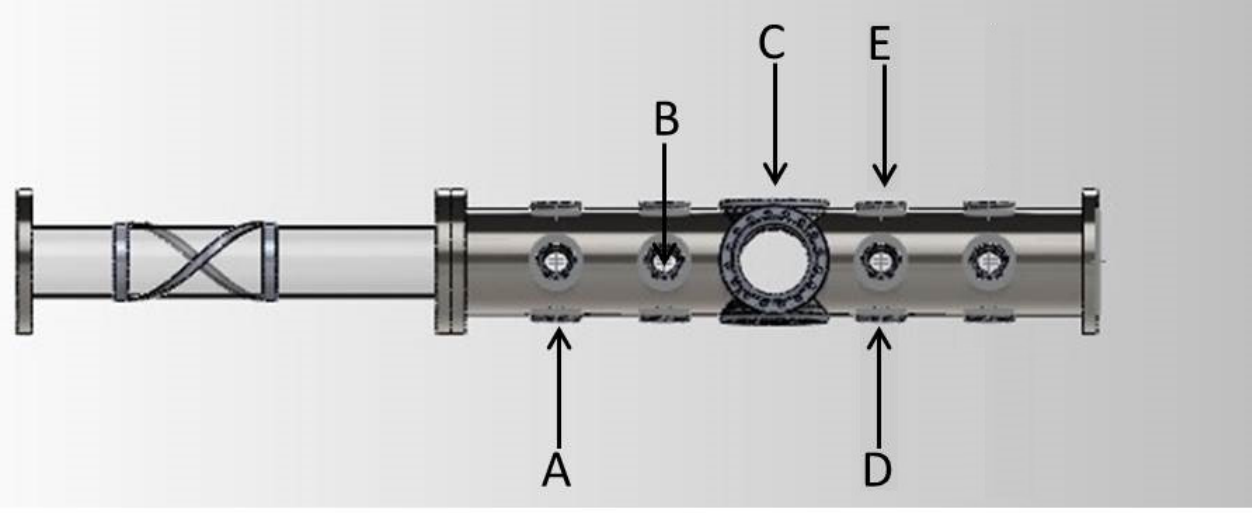

Figure 3.4: Top-view schematic of the HELIX chamber (electromagnets removed for clarity) with labels corresponding to the diagnostic elements used in this work. (A) is the location of the internal wave-launching antenna; (B) is the location of the magnetic sense coil probe $(15 \mathrm{~cm}$ from the wavelaunching antenna), which was inserted from the top of the chamber; (C) is the location of the LIF diagnostic (30 $\mathrm{cm}$ from the wave-launching antenna), with laser light injected from the side (perpendicular to the axial magnetic field) and collected from the top of the chamber, and (D) and (E) are the locations where time-resolved Langmuir probe measurements were made (45 $\mathrm{cm}$ from the wave-launching antenna). 
attaches to a turbomolecular pumping station, an ion gauge, and a 12" stainless steel flange fitted with a 4" viewport for LIF diagnostic access, while the other end is connected to LEIA. The HELIX chamber itself consists of a Pyrex tube $10 \mathrm{~cm}$ in diameter and $61 \mathrm{~cm}$ in length where the plasma is generated (connected to the glass cross) and a stainless steel chamber $15 \mathrm{~cm}$ in diameter and $91 \mathrm{~cm}$ long (connected to LEIA). The stainless steel segment of HELIX has diagnostic access via one set of four 6" Conflat ${ }^{T M}$ crossing ports at the middle of the chamber and four sets of four $2 \frac{3}{4}$ " Conflat $^{T M}$ crossing ports equally-spaced on either side. The end of the stainless steel tube not connected to the Pyrex tube is connected to LEIA, which is a 1.8 $\mathrm{m}$ diameter, $4.4 \mathrm{~m}$ length expansion chamber. The other end of the LEIA expansion chamber is connected to a turbomolecular pumping station. All of the experiments described in this thesis were conducted in the HELIX source region running in pulsed mode (an $80 \mathrm{~ms}$ long plasma pulse repeated every two seconds) with the LEIA electromagnets turned off (see Fig. 3.4).

The axial magnetic field in HELIX is supplied by ten water-cooled electromagnets. Up to $400 \mathrm{~A}$ of current is supplied by two Xantrex XFR power supplies connected in parallel. The maximum attainable field is $1.2 \mathrm{kG}$. All measurements in this work were made at $800 \mathrm{G}$. The axial magnetic field 
in LEIA is supplied by seven nine-foot-diameter electromagnets. Each electromagnet consists of 20 turns of water-cooled hollow rectangular aluminum tubing. Up to $200 \mathrm{~A}$ of current is provided by an EMI DC power supply with a maximum achievable field of approximately $140 \mathrm{G}$.

The power for plasma generation in HELIX is supplied by a $50 \mathrm{MHz}$ Wavetek model-80 function generator connected to an ENI A1000 $300 \mathrm{kHz}-$ $35 \mathrm{MHz} 1 \mathrm{~kW}$ RF power amplifier. The amplifier output is connected through a high-frequency coaxial cable to a $\pi$-type matching network that matches the impedance of the plasma/antenna/network impedance to the $50 \Omega$ output impedance of the amplifier. The matching network consists of one tunable 20-2000 pF Jennings high-voltage vacuum capacitor, the load capacitor, and three smaller (two 2-350 pF capacitors and one 5-500 pF capacitor) Jennings high-voltage vacuum capacitors, the tuning capacitors. The load capacitor is in series with the three tuning capacitors, which are all in parallel with each other, as shown in Fig. 3.5. A detailed description of the matching network can be found in Balkey. ${ }^{[60]}$ The RF power is coupled into the plasma via a 19 $\mathrm{cm}$, half wave, $m=+1$ helical antenna (see Fig. 3.6) wrapped tightly around the Pyrex tube approximately $37 \mathrm{~cm}$ from the end connected to the pumping station. 


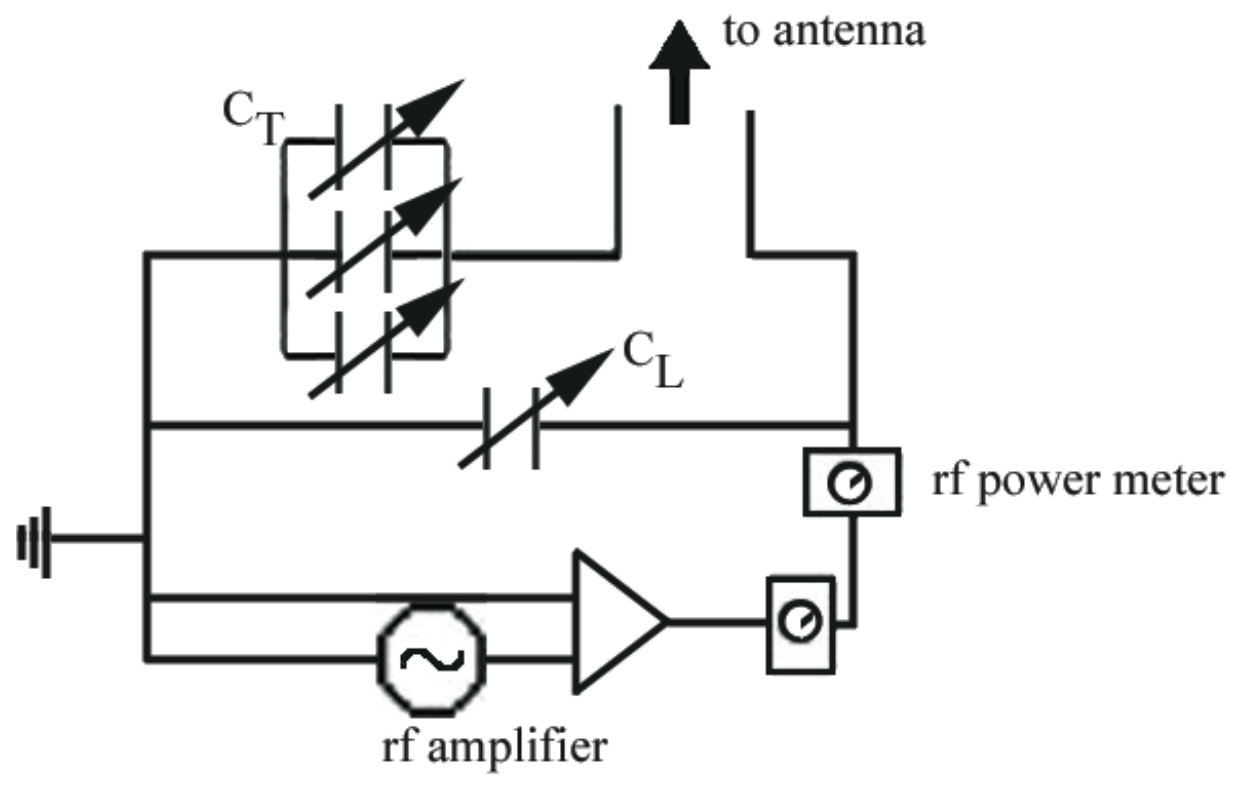

Figure 3.5: The impedance matching network for coupling power into HELIX. In the diagram, $\mathrm{C}_{L}$ is the load capacitor and $\mathrm{C}_{T}$ is the set of three tuning capacitors. The matching network is connected to the antenna by two copper bars which are enclosed in a plastic teflon pipe for safety.

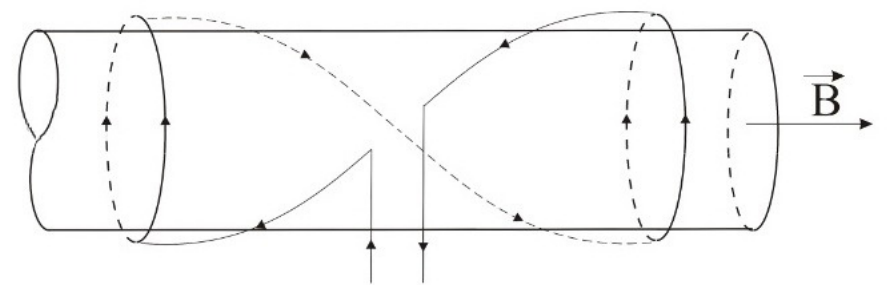

Figure 3.6: The $m=+1$ helical antenna used in these experiments. Figure adapted from Biloiu. ${ }^{[61]}$ 


\begin{tabular}{|c|c|}
\hline Plasma Parameter & Typical Value in HELIX \\
\hline \hline Gas Species & Argon, Helium, Nitrogen \\
\hline Base Pressure & $<1.3 \times 10^{-7}$ Torr \\
\hline Operating Pressure & 0.1 to $20 \mathrm{mTorr}$ \\
\hline Magnetic Field & $\leq 1.2 \mathrm{kG}$ \\
\hline RF Power & 0 to $1 \mathrm{~kW}$ \\
\hline Operating Frequency & $6-18 \mathrm{MHz}$ \\
\hline Density & $\leq 3 \times 10^{13} \mathrm{~cm}^{-3}$ \\
\hline Ion Temperature & $\leq 1 \mathrm{eV}$ \\
\hline Electron Temperature & $\sim 3-5 \mathrm{eV}$ \\
\hline Ion Gyro-Radius & $\sim 2.7 \mathrm{~mm}$ \\
\hline Electron Gyro-Radius & $\sim 0.04 \mathrm{~mm}$ \\
\hline
\end{tabular}

Table 1: Characteristic plasma parameters for HELIX. 


\subsection{Pulsed Experiment Timing}

In the wave-launching experiments discussed here, the plasma was run in pulsed, rather than continuous-wave $(\mathrm{CW})$, mode. Pulsing the experiment allowed us to keep the internal wave-launching antenna and magnetic sense coil probe from burning up in the hot, dense plasma. Each plasma pulse was tens of ms long (60 ms long for magnetic sense coil and LIF measurements, $80 \mathrm{~ms}$ long for Langmuir probe measurements) and repeated at 2-second intervals. The 2 -second repetition rate was chosen because it was the fastest rate at which a high-density core reliably and reproducibly formed for every pulse; trying to pulse the experiment faster at high pressure and magnetic field values led to frequent pulses without a high-density core. Pulses without a high-density core (and, therefore, no steep density gradient) were not conducive to launching Alfvén waves and made averaging multiple shots together to improve the signal-to-noise ratio untenable.

Since the LIF measurements were averaged over 100 individual shots for each wave point (and each LIF scan was made up of 64 individual wave points over a $10 \mathrm{GHz}$ range), the shortest LIF data sets took close to 9 hours to collect, since both wave-on and wave-off measurements were needed in order to observe any temperature difference due to the driven wave perturbation. 
On a typical day when laser stability was not ideal, a complete LIF data set (wave-on and wave-off cases at a single radial location) took between 18 and 25 hours. Most LIF data sets took between 14 and 16 hours to acquire.

The beginning of each plasma pulse (approximately the first 15-20 ms) showed a transient start-up phase during which the argon gas was not yet fully dissociated into plasma. The launched wave pulse (which is $8 \mathrm{~ms}$ long, or 200 cycles of the $25 \mathrm{KHz}$ driven wave) was delayed until $32.5 \mathrm{~ms}$ into the plasma pulse to ensure the perturbation interacted with steady-state rather than transient plasma. 


\section{Chapter 4}

\section{Magnetic Sense Coil Probes}

\subsection{Magnetic Sense Coil Probe Theory and Design}

Measurements of the fluctuating magnetic field in these experiments were made using a magnetic sense coil probe (commonly referred to as a "b-dot" probe because the induced voltage change across the magnetic sense coil is directly proportional to the time derivative of the magentic flux passing through the plane of the coil). Using Faraday's Law of Induction, the potential drop through a loop (or series of loops) of small-gauge wire can be related to the temporal change in magnetic flux at the spatial location of the probe.

$$
\nabla \times \mathbf{E}=-\frac{\partial}{\partial t} \mathbf{B}=-\dot{\mathbf{B}}
$$

The change in magnetic flux through a surface area $d A$ is calculated as:

$$
\int d \mathbf{A} \cdot(\nabla \times \mathbf{E})=-\int d \mathbf{A} \cdot \dot{\mathbf{B}}
$$


Applying Stokes theorem to Eqn. 4.2 yields

$$
\oint_{C} d \mathbf{l} \cdot \mathbf{E}=-\int d \mathbf{A} \cdot \dot{\mathbf{B}} .
$$

Therefore, for a probe with $N$ turns whose surface normal is oriented at an angle $\theta$ with respect to the magnetic field, the potential drop across the probe coil is,

$$
-\Delta V=N A \dot{B} \cos \theta
$$

From Eq. 4.4, the measured fluctuations in $\Delta V$ on the probe are directly related to the temporal magnetic field fluctuations.

\subsection{Magnetic Sense Coils Used in These Experiments}

\subsubsection{Commercial Inductor Coils}

Based on previous Alfvén propagation studies in a helicon plasma by

Watts and Hanna ${ }^{[17],[18]}$, the first magnetic sense coil probe used in this work was constructed from a commercially available surface-mount inductor. Unlike in the Watts and Hanna work, water cooling for the probe was not an 
option, so the plasma was run in pulsed, rather than continuous, mode to prevent heat damage to the inductor and electrical connections. For this work, a Coilcraft $1 \mathrm{mH}, 44 \Omega$ inductor (model 1812LS-105XJLB) was used to construct the magnetic sense coil probe. A twisted pair cable was soldered the end plates of the inductor and Torr seal epoxy applied to insulate those connections. A thin copper foil was wrapped around the inductor and the twisted pair to provide electrostatic shielding, and then the whole assembly was inserted into a $8 \mathrm{~mm}$ OD clear quartz tube to insulate it from the plasma. While Watts and Hanna reported sensitivity good enough to measure changes in the perturbation amplitude and phase across the plasma column, this technique did not seem to work for collecting data in HELIX. Being unable to reliably measure wave perturbations with a commercial inductor, we decided to replace the commercial inductor coil with a hand-wound coil for increased sensitivity.

Switching from a commercially available surface-mount inductor to a hand-wound coil was the first step in improving our experimental measurements. This was done before any modifications to the wave-launching antenna were made (see Chapter 5). Since the same hand-wound 50-turn coil was used to measure field perturbations for all three antenna designs, it is 
very likely that the original magnetic sense coil probe design was completely functional. Since later modification of the wave-launching antenna led to the conclusion that the original antenna design did not couple power into the plasma very well, it would seem that the low-power coupling of the original external antenna design was the problem all along and that magnetic sense coil probes constructed from small commercially available surface-mount inductors could be an option for future work involving an array of magnetic sense coil probes.

\subsubsection{Hand-Wound Inductor Coils}

While the largest commercial inductor which could fit inside the $8 \mathrm{~mm}$ OD quartz tubes used for shielding only had about 30 turns (estimated empirically since the manufacturer considers number of turns used in their inductors proprietary information), a hand-wound coil made with size 35 magnet wire with a similar collection area could still fit inside the quartz tube with 50 total turns. Since the sensitivity of the magnetic sense coil probe scales with the number of turns in the coil, as shown in Equation 4.4, this was an increase in sensitivity of approximately $67 \%$.

The first step in construction was cutting a cylindrical macor form to 
a length that would fit inside the $8 \mathrm{~mm}$ OD quartz tube (about $3 \mathrm{~mm}$ ). The macor form was then held in place by an alligator clip under a lighted magnifying lens while 50 coils of the magnet wire were wrapped around it. By wrapping 2 layers of turns, 25 turns per layer, around the cylindrical form, the collecting solenoid of the magnetic sense coil probe was constructed. A very small amount of Torr Seal epoxy was applied across the turns of the first layer in order to hold them in place while the second layer was wound onto the form. After all 50 turns were in place, another thin layer of epoxy was applied across the outer layer of coils to hold everything together. The loose ends of the magnet wire (measured out before the winding took place to be able to run the entire length of the probe shaft) were carefully twisted together to make a twisted pair cable. This cable was fed through a stainless steel probe shaft and the ends were soldered to two pins on the inside of a hermetically sealed vacuum BNC electrical feedthrough. To shield the magnetic sense coil probe from electrostatic pick-up, it was enclosed inside a grounded conductor. The twisted pair in the probe shaft was surrounded by a mesh wire shield in continuity with the chamber ground, while the magnetic sense coil and the $9 \mathrm{~cm}$ of twisted pair cable closest to it were coated in several layers of conducting silver paint. A small length of magnet wire was soldered to the 


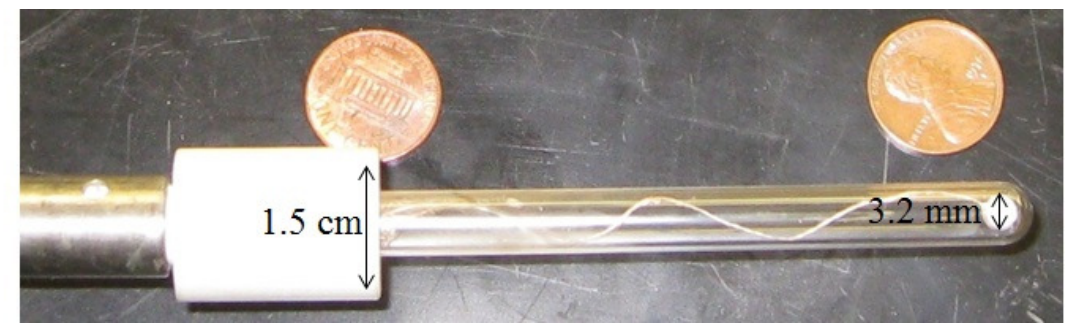

Figure 4.1: Picture of the hand-wound magnetic sense coil probe with pennies shown for scale. The probe is made of 50 turns of size 35 magnet wire in two layers (25 turns per layer) wrapped around a macor cylinder. The coil and leads are covered in conducting silver paint to shield the probe from electrostatic pick-up, and the whole thing is insulated by an $8 \mathrm{~mm}$ OD quartz tube which is affixed to the machined, threaded macor probe-shaft adapter with a set screw. The silver paint is in contact with a woven mesh wire shield which runs the length of the probe shaft and is continuous with ground while the coil and leads are fully insulated from ground.

mesh shield and wrapped tightly around the last half centimeter of the silver paint to ensure that the entire conducting shield was in continuity and held at the same ground.

Measurements with the magnetic sense coil probe were taken at quartercentimeter steps across the plasma column. Typical single time series at these radial steps are shown in Figure 4.2. The large magnitude signal seen at early times $(t<10 \mathrm{~ms})$ is due to the transient nature of the plasma start-up phase. For analysis of these magnetic sense coil probe signals, see Chapter 6 . A set of high-speed differential signal amplifiers designed by the UW-Madison MST group made the magnetic sense coil probe measurements possible. The 
amplifiers have three parallel outputs with cutoff frequencies of $215 \mathrm{kHz}, 615$ $\mathrm{kHz}$, and 1.9 MHz. Each channel can be set to a selectable gain from 0.1 to 50. For the measurements presented here, the magnetic sense coil probe signal was connected to the $215 \mathrm{kHz}$ output channel with a gain of 50 . 

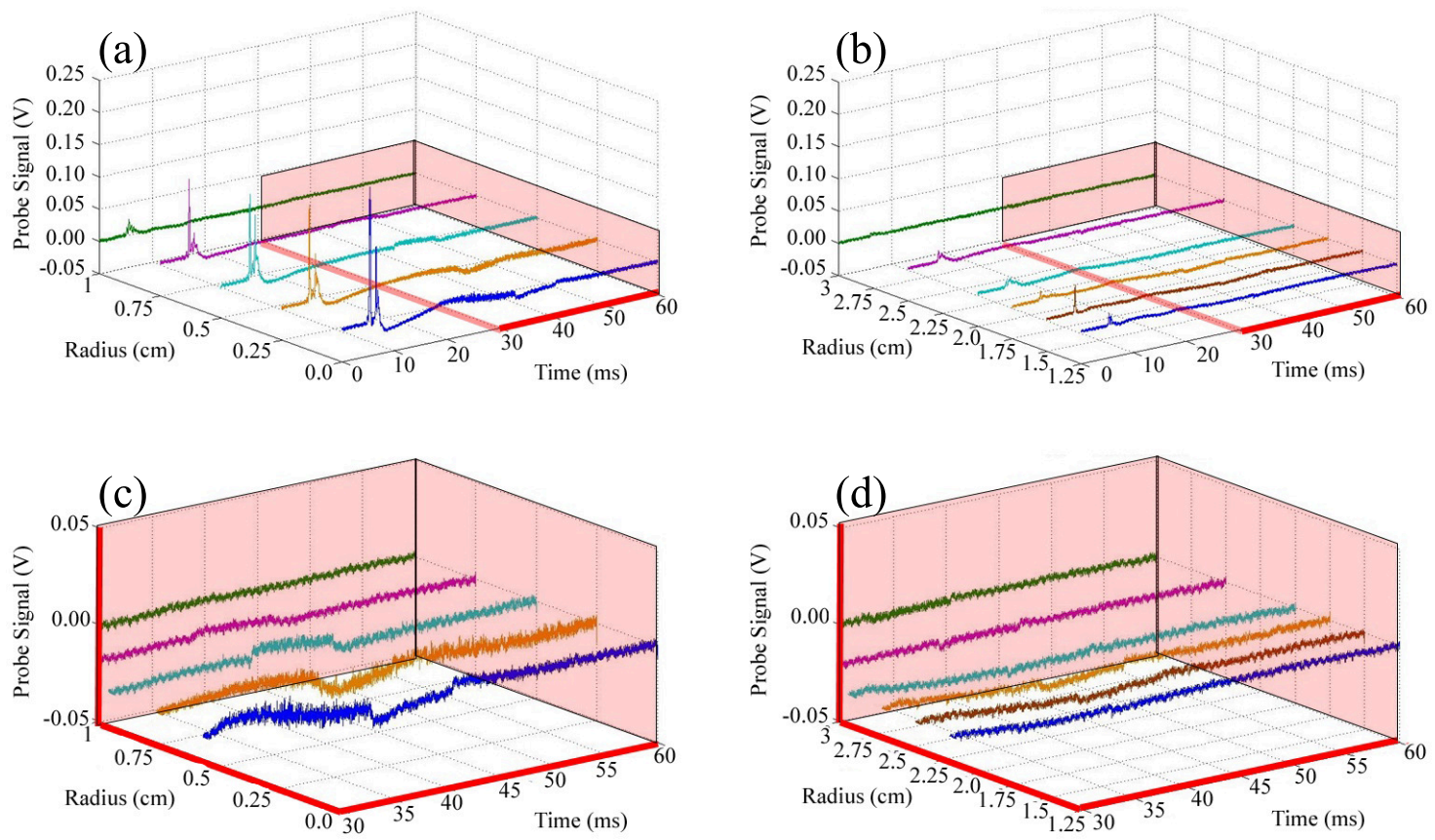

Figure 4.2: Typical magnetic sense coil probe time series for quartercentimeter radial steps across the plasma column. (a) Shows the full time series for the radial values $r=0 \mathrm{~cm}$ (blue), $r=0.25 \mathrm{~cm}$ (orange), $r=0.5 \mathrm{~cm}$ (aqua), $r=0.75 \mathrm{~cm}$ (magenta), and $r=1 \mathrm{~cm}$ (green) while (c) shows a smaller time window at the same locations (same colors) when the wave perturbation is turned on. (b) Shows the full time series for the radial values $r=1.25 \mathrm{~cm}$ (blue), $r=1.5 \mathrm{~cm}$ (brown), $r=1.75 \mathrm{~cm}$ (orange), $r=2 \mathrm{~cm}$ (aqua), $r=2.5 \mathrm{~cm}$ (magenta) and $r=3 \mathrm{~cm}$ (green) while (d) shows a smaller time window at the same locations (same colors) when the wave perturbation is turned on. 


\section{Chapter 5}

\section{Wave Launching Antennas}

\subsection{External Wave-Launching Antenna Design}

Building on previous resonant ion heating work conducted by John Kline ${ }^{[62]}$ in the HELIX/LEIA system, the first wave-launching antenna design employed for this experiment was a rectangular transverse antenna external to the plasma chamber. While the principle was the same as that in Kline's work (two rectangular coils on opposite sides of the Pyrex tube such that the magnetic field driven by the two coils of wire create a time-dependent magnetic field perturbation transverse to the axial magnetic field), the design had to be modified to accommodate limitations not faced in Kline's work. While the current HELIX chamber consists of a 61-cm-long Pyrex tube connected to a 91-cm-long stainless steel tube (with a total of 5 sets of crossing diagnostic ports, see Fig. 3.4), the HELIX chamber at the time of Kline's work was a single 157-cm-long Pyrex tube with a single set of crossing diagnostic ports. The new configuration provides much more versatile diagnostic access, 


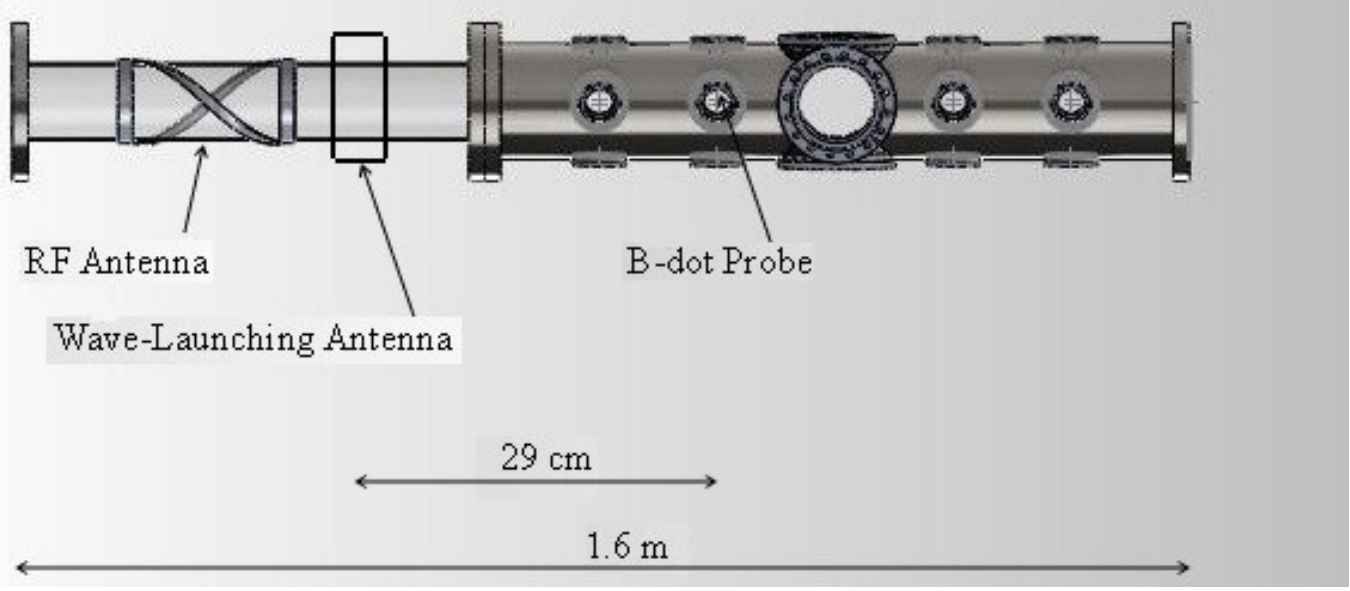

Figure 5.1: Side view of HELIX with the positions of the rectangular transverse antenna and magnetic sense coil probe indicated.

but severely limits the space available for an external ion-heating antenna.

Kline was able to use a 55-cm-long, 20-cm-high rectangular transverse antenna for resonant ion heating, while the current HELIX configuration only has enough space for a 7-cm-long, 15-cm-high rectangular transverse antenna (see Fig. 5.1).

Wave-launching was attempted in both helium (driving frequency at 275 $\mathrm{KHz}$ to stay below the ion-cyclotron frequency), building on Houshmandyar's previous Alfvén wave work in HELIX ${ }^{[3]}$; and argon (driving frequency at $25 \mathrm{KHz}$ to stay below the ion-cyclotron frequency) plasmas, but with no 
observed perturbations in the plasma core or density gradient. Magnetic sense coil probe measurements made in radial steps across the plasma column indicated some very small azimuthal perturbations at the edge of the plasma only. Without the ability to excite magnetic field perturbations in the highdensity core due to the spatial limitations of this antenna, we abandoned that external transverse antenna design and moved on to an internal antenna.

\subsection{Internal Wave-Launching Antenna Designs}

\subsubsection{Hand-Wound Transverse Antenna}

Although the external rectangular transverse antenna did not generate strong enough magnetic field perturbations to penetrate the high-density core, it was still capable of perturbing the edge of the plasma column, i.e., the plasma closest to the antenna. With that in mind, an internal transverse antenna was constructed. This antenna needed to be small enough to

fit through one of the $2 \frac{3}{4}$ " Conflat $^{T M}$ ports in the stainless steel section of HELIX. Although this required an even smaller antenna than the previous design, the fact that the end of the antenna (where the transverse field perturbations would be strongest) would be inside the plasma column and at 


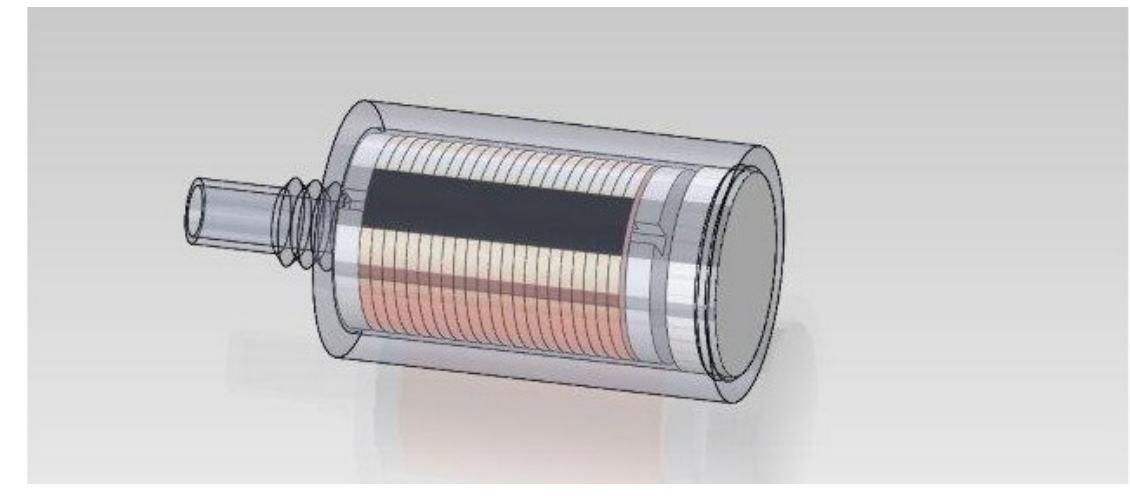

Figure 5.2: Schematic representation of the internal transverse antenna. The antenna has an outer diameter of $3 \mathrm{~cm}$ and a total length of $5 \mathrm{~cm}$. The shielding is stainless steel that was covered in a spray-on alumina compound to insulate it from the plasma. The antenna itself is 29 turns of 14-gauge wire wrapped around a machined macor form. The antenna coil itself is 3.4 cm long.

the edge of the high-density core was promising. This internal antenna was made as large as possible given the size constraints of the $2 \frac{3}{4}$ " Conflat $^{T M}$ port available for access to the plasma (see Fig. 5.2).

Ultimately, despite the internal circular transverse antenna being directly next to the high-density core, the design suffered from the same limitations as the external rectangular transverse antenna. The magnitude of maximum magnetic perturbation possible given the size constraints of the system was insufficient to couple meaningfully into the plasma. With insufficient field perturbations provided by transverse antennas, the design was abandoned altogether and a new method of transverse field perturbation was constructed. 


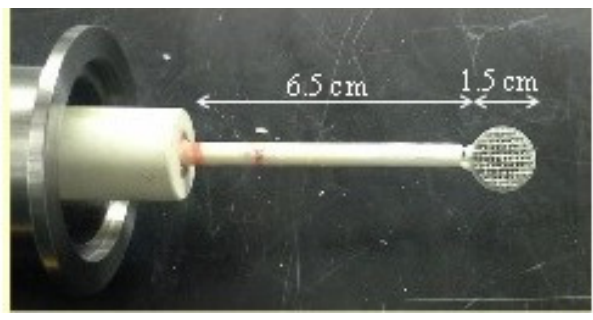

Figure 5.3: Internal mesh grid antenna for setting up a modulated current channel to generate an azimuthal magnetic field perturbation.

\subsubsection{Conducting Mesh Grid Antenna}

With magnetic field perturbations from loop antennas too small to couple into the plasma, a new design was developed. Instead of using a loop antenna to drive magnetic field perturbations, a mesh grid antenna (see Fig. 5.3) was used to set up a current channel in the plasma. This current channel acted as a current-carrying wire with an azimuthal magnetic field. By modulating the bias on the grid antenna at $25 \mathrm{KHz}$, the current channel and therefore the azimuthal magnetic field associated with it could be modulated at $25 \mathrm{KHz}$. In this way a $25 \mathrm{KHz}$ azimuthal magnetic field perturbation was introduced to the plasma at the edge of the high-density core.

To drive current in the plasma, a 200-cycle burst of a $25 \mathrm{kHz}$ sine wave was applied to the grid antenna for $8 \mathrm{~ms}$ starting at $32.5 \mathrm{~ms}$ into the plasma discharge; ensuring that the transient plasma generation stage had settled down and the plasma was in steady-state before the perturbation was launched. 
The antenna current signal is shown in Figure 5.4. It is important to notice that early in the plasma discharge $(t<10 \mathrm{~ms})$ there is an induced current signal on the mesh grid due to the plasma start-up phase. Depending on the exact configuration of the plasma matching network, this early plasma behavior can be controlled and changed, with different early behavior arising from different plasma conditions and possibly different plasma responses to the perturbation signal. To ensure that measurements made during different data sets were comparable, the matching network was adjusted in small increments at the beginning of every data collection period to induce the same early-time behavior in the plasma, and therefore, the same plasma response to the wave perturbation.

In this work a Wavetek $50 \mathrm{MHz}$ function generator model 80 was used to generate a burst sine wave triggered by the same transistor-transistor logic (TTL) circuit pulse that triggered the plasma discharge. A time delay op-amp circuit was placed in-line with the pulse generator to ensure that the wave burst did not start until $32.5 \mathrm{~ms}$ into the discharge. The output of the Wavetek was connected to a Kepco Bipolar Operational Power Supply/Amplifier which was used to drive the perturbing current on the mesh grid wave-launching antenna. 


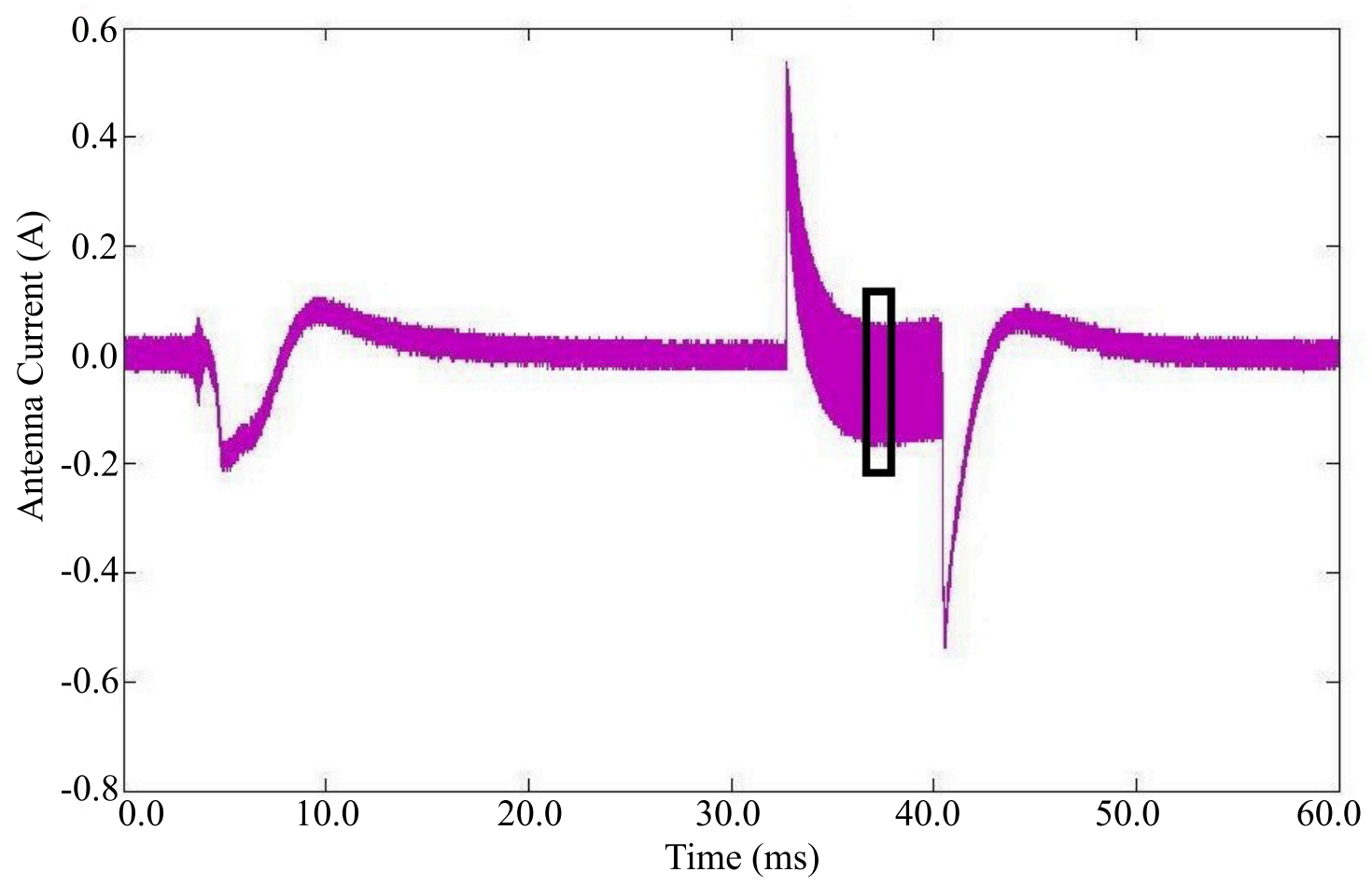

Figure 5.4: The measured current signal on the wave-launching antenna during a complete plasma discharge. The antenna current is measured with a $1 \mathrm{~V}=1 \mathrm{~A}$ Rogowski coil fitted around one of the antenna leads between the output of the Kepco Bipolar Amplifier and the vacuum feedthrough. 


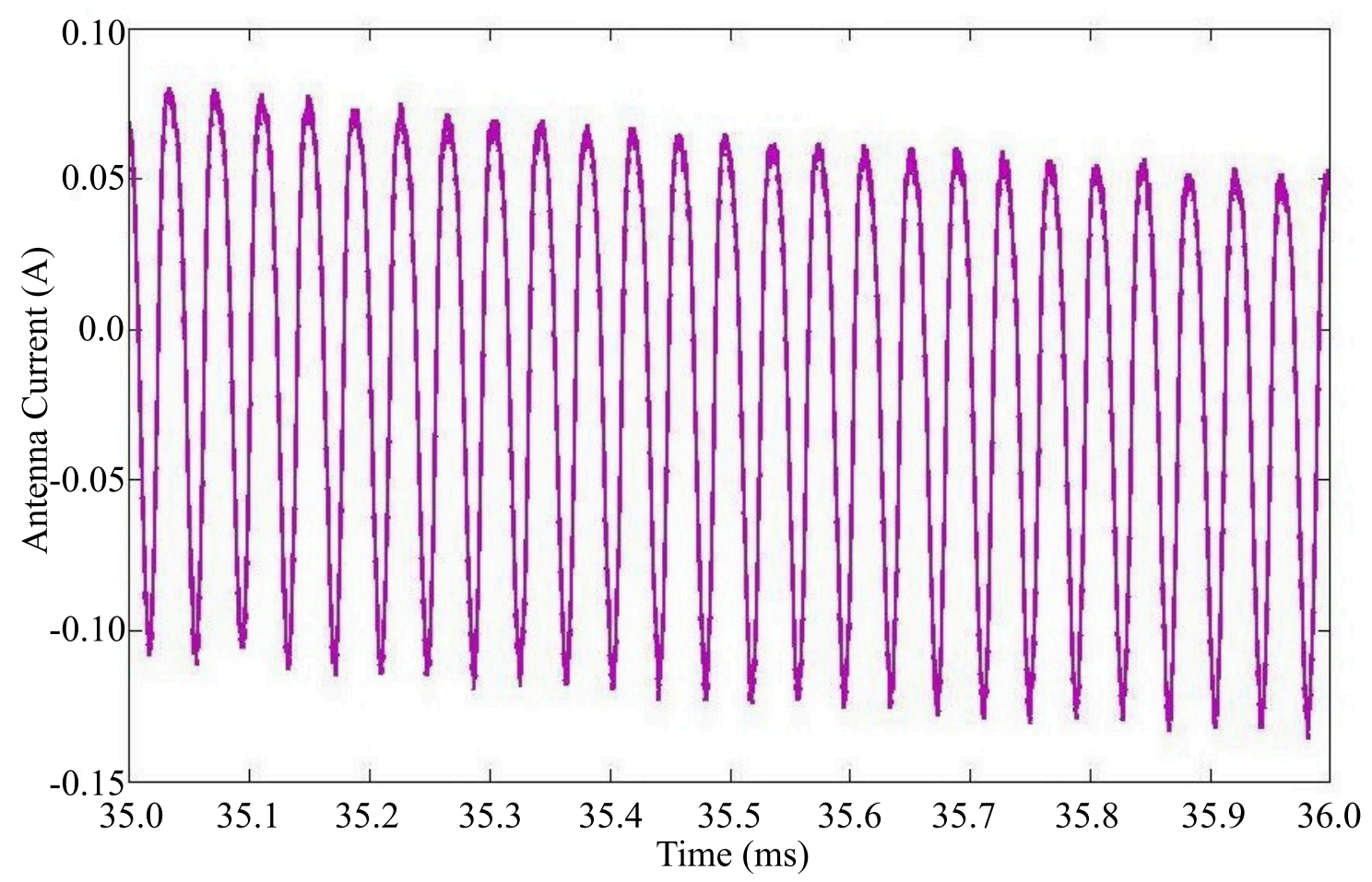

Figure 5.5: A $1 \mathrm{~ms}$ time window of the perturbation pulse (the black box shown in Figure 5.4), from $t=35 \mathrm{~ms}$ to $t=36 \mathrm{~ms}$, showing the $25 \mathrm{kHz}$ perturbation signal. The driving signal has a maximum amplitude of $100 \mathrm{~mA}$, which when used in the formula for the magnetic field due to a currentcarrying wire $\left(B=\mu_{0} I / 2 \pi r\right)$, corresponds to a maximum field perturbation of $\sim 2 \mathrm{G}(\sim 0.25 \%$ of the background field $)$. 


\section{Chapter 6}

\section{Magnetic Probe Measurements}

\subsection{Steady-State Density Measurements and Perpendicular Length Scales}

As discussed in Chapter 2.1, when the perpendicular wavelength becomes comparable to the electron skin-depth, perpendicular dynamics play a role in the parallel wave propagation. At the densities measured in this work, the electron skin-depth is on the order of $1-2 \mathrm{~cm}$. Since the high density core is on this scale, it is reasonable to expect the perpendicular wavelength bounded by the core to play a role in the propagation of the launched waves. ${ }^{[4]}$ Figure 6.1 shows the density profile in HELIX as well as the density gradient scale length, $n / \nabla n$, (see Chapter 8 for detailed discussion of Langmuir probe density measurements) across the plasma column. For $r<1 \mathrm{~cm}$, the density gradient scale is close to $0.25 \mathrm{~cm}$. Between $r=1 \mathrm{~cm}$ and a little more than $r$ $=1.25 \mathrm{~cm}$, the density gradient scale increase to a little over $2 \mathrm{~cm}$, and then drops back to 0.25 as the plasma density drops at larger radii. From this we 
expect to see a change in the parallel wavelength with radius. By measuring the phase angle between the launched antenna signal and the magnetic sense coil probe signal, the wavelength of the launched perturbation can be measured and compared to the prediction from the full dispersion relation. This phase angle measurement and wavelength comparison is discussed in Section 6.2.

\subsection{Phase Angle Measurements}

By using the cross power spectrum of two measured time series, it is possible to determine the phase difference between them for a specific frequency. In this work, the cross power spectrum between the sinusoidal perturbation signal applied to the wave-launching antenna and the sinusoidal signal detected by the magnetic sense coil probe is used to determine the phase angle (and therefore, the wavelength) of the propagating wave at the location of the magnetic sense coil probe. The cross power spectrum of two time series is defined as the inner product of the Fourier transform (FT) of one time series with the complex conjugate of the FT of the second time series. If we represent the two time series under consideration as $f_{1}\left(x_{1}, t\right) f_{2}\left(x_{2}, t\right)$, then 


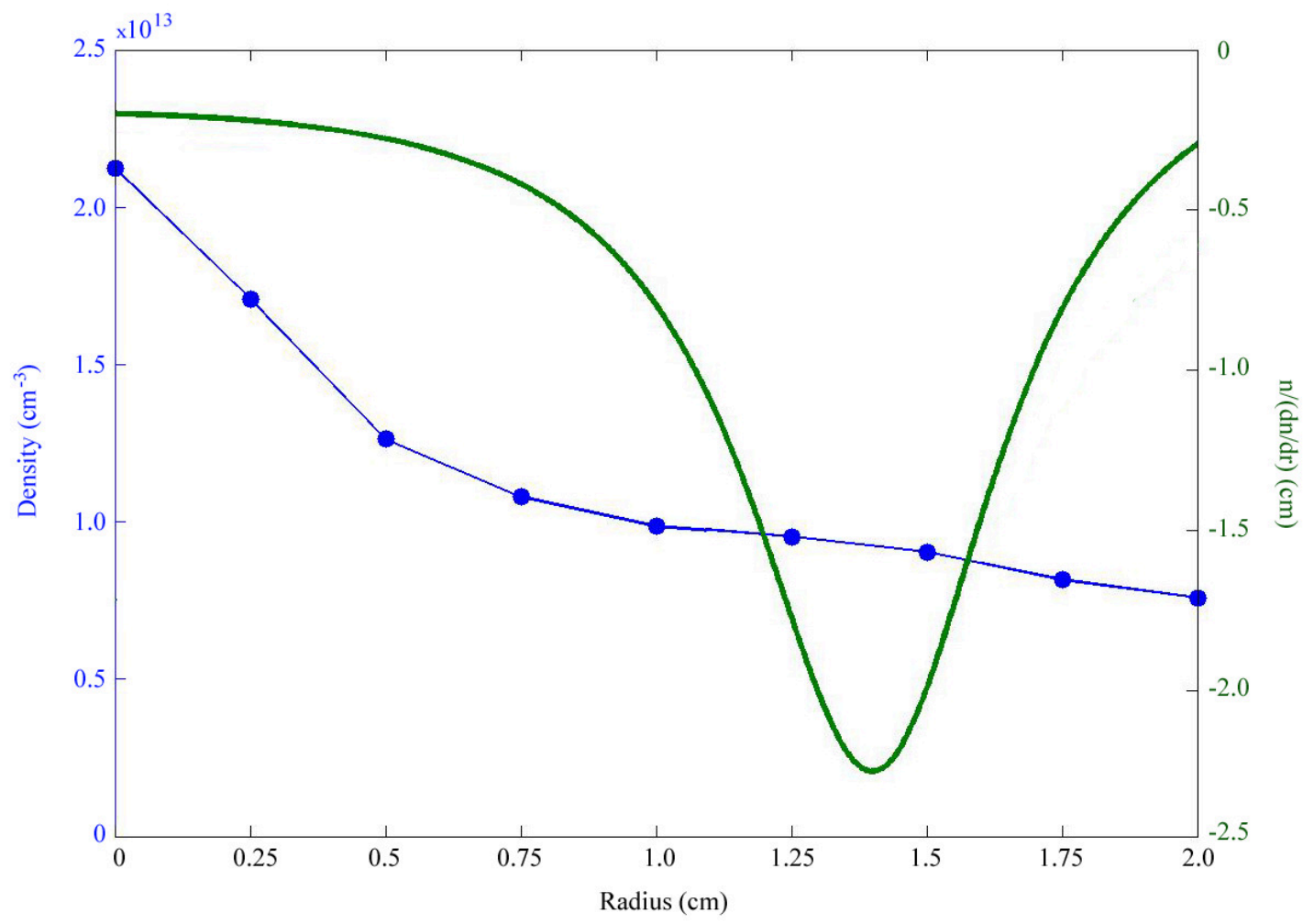

Figure 6.1: Steady-state electron density measurements taken over quarter centimeter steps are shown in blue. The green curve shows $n / \nabla n$. The high density core is contained within a half-centimeter radius and the steepest density gradient is localized between $r=0.75 \mathrm{~cm}$ and $r=1.25 \mathrm{~cm}$. 
the two Fourier transforms to consider are

$$
\Phi_{1}\left(x_{1}, \omega\right)=\int_{-\infty}^{\infty} f_{1}\left(x_{1}, \omega\right) \cos (\omega t) \mathrm{d} t \int_{-\infty}^{\infty} f_{1}\left(x_{1}, \omega\right) \sin (\omega t) \mathrm{d} t
$$

and

$$
\Phi_{2}\left(x_{2}, \omega\right)=\int_{-\infty}^{\infty} f_{2}\left(x_{2}, \omega\right) \cos (\omega t) \mathrm{d} t \int_{-\infty}^{\infty} f_{2}\left(x_{2}, \omega\right) \sin (\omega t) \mathrm{d} t
$$

where $\mathrm{x}_{1}$ and $x_{2}$ are the locations where each signal is being measured. The cross power spectrum, $P_{12}$, of the signals $f_{1}$ and $f_{2}$ is the product of Equation 6.1 and the complex conjugate of Equation 6.2, which can be written compactly as

$$
P_{12}(d, \omega)=\Phi_{1}\left(x_{1}, \omega\right) \Phi_{2}^{*}\left(x_{2}, \omega\right)
$$

In Equation 6.3, $d=x_{1}-x_{2}$ is the physical distance between the location where $f_{1}$ is measured and the location where $f_{2}$ is measured. Equation 6.3 can be expanded and the real and imaginary terms collected to yield: 


$$
\begin{aligned}
P_{12}(d, \omega)= & \left(\operatorname{Re}\left\{\Phi_{1}\right\} \operatorname{Re}\left\{\Phi_{2}\right\}+\operatorname{Im}\left\{\Phi_{1}\right\} \operatorname{Im}\left\{\Phi_{2}\right\}\right) \\
& +i\left(\operatorname{Im}\left\{\Phi_{1}\right\} \operatorname{Re}\left\{\Phi_{2}\right\}-\operatorname{Re}\left\{\Phi_{1}\right\} \operatorname{Im}\left\{\Phi_{2}\right\}\right) .
\end{aligned}
$$

The phase difference between the two times series is the same as the angle between the real and imaginary vectors of the cross power spectrum plotted in the complex plane:

$$
\Theta(\omega)=\tan ^{-1}\left(\frac{\operatorname{Im}\left\{\Phi_{1}\right\} \operatorname{Re}\left\{\Phi_{2}\right\}-\operatorname{Re}\left\{\Phi_{1}\right\} \operatorname{Im}\left\{\Phi_{2}\right\}}{\operatorname{Re}\left\{\Phi_{1}\right\} \operatorname{Re}\left\{\Phi_{2}\right\}+\operatorname{Im}\left\{\Phi_{1}\right\} \operatorname{Im}\left\{\Phi_{2}\right\}}\right) .
$$

For this technique to be feasible, a large signal to noise ratio is required. If the signal to noise ratio is not large enough, averages of cross power spectra can be used to improve the precision of the phase angle measurements. In these measurements, the random error decreases as $1 / \sqrt{M}$ where $M$ is the number of samples. ${ }^{[63]}$ To handle the large data records necessary to make this technique useful, a LeCroy WaveRunner ${ }^{\mathrm{TM}}$ 604Zi digital oscilloscope with the capacity to perform onboard averages of fast Fourier transforms (FFTs) was used. Fourier analysis of the discrete signals is accomplished through the discrete Fourier transform (DFT) and the FFT is a recursive algorithm 


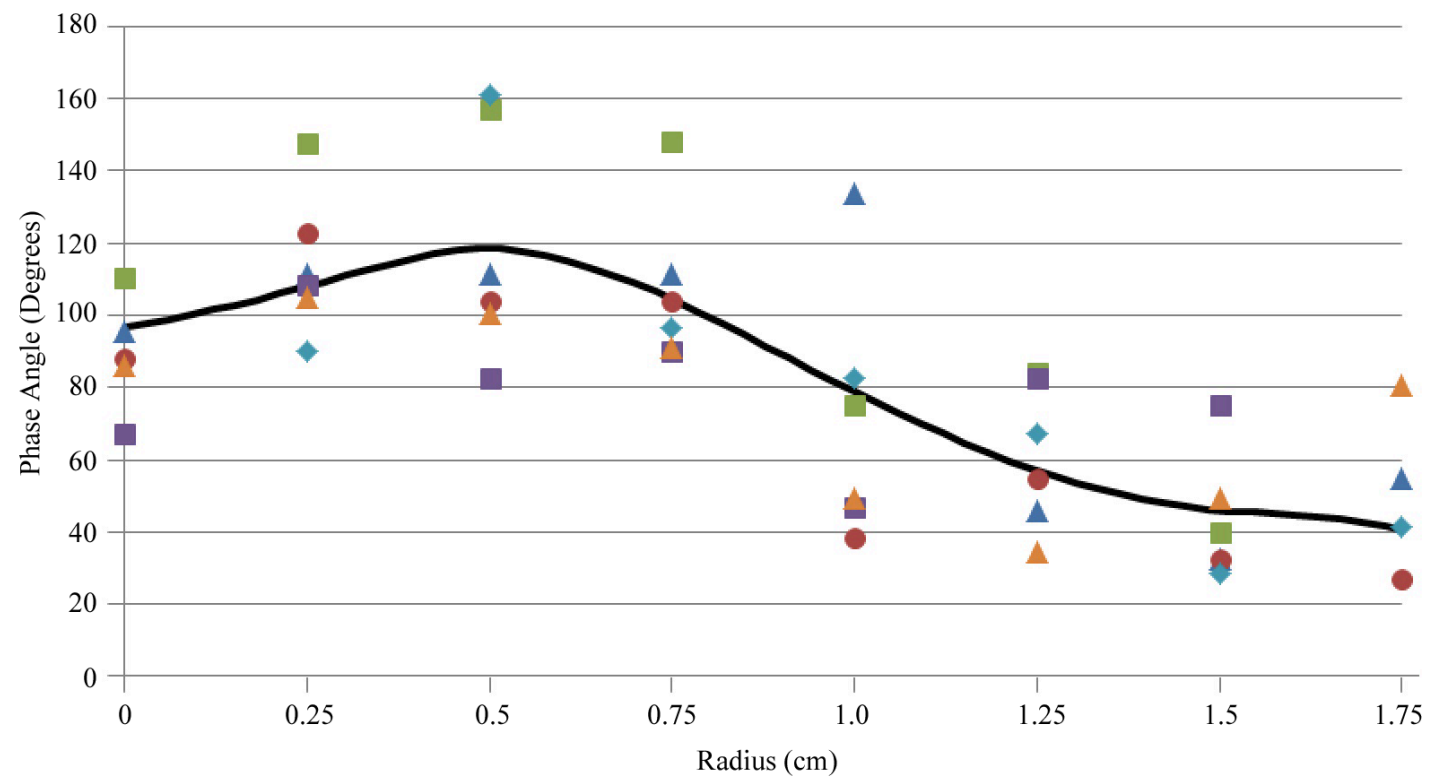

Figure 6.2: Phase angle measurements for six different data sets. The average phase behavior across the plasma column is shown by the solid black line. The spread of phase angle values at each spatial location demonstrate the significant variation in parallel wavelength. Future measurements of the distribution of this spread are necessary and, if they are consistent with a turbulent spectrum, could demonstrate plasma turbulence.

which implements DFTs more efficiently. A more detailed discussion of DFTs and FFT can be found in Section 6.3. For this work, the oscilloscope was programmed to record the time series of both the antenna current signal and the magnetic sense coil probe signal, calculate the cross power spectrum of the two signals, and save the ratio of the imaginary component to the real component for later computation of the phase angle at each radial location in the plasma column. 
Phase angle measurements across the plasma column are shown in Figure 6.2. The qualitative behavior is consistent across six different data sets. The spread in phase angle indicates that turbulence might be present during the perturbation pulse. The presence of a turbulent spectrum is corroborated by the wavelet analysis of the magnetic sense coil probe signal (see Section 6.3.3).

From the measured phase angle and the separation between the wavelaunching antenna and the magnetic sense coil probe $(15 \mathrm{~cm})$, the parallel wavelength is calculated. The Alfvén wavelength calculated from linear theory is nearly five times larger than the wavelength determined from the phase angle measurements. When the linear theory is modified to account for perpendicular wavelength (using the density gradient scale length) and finite frequency effects (see Chapter 2.1 for derivation and discussion of the modified dispersion relation), the wavelength from the phase angle measurements is in reasonable agreement with the wavelength predicted by the inertial Alfvén wave dispersion relation (see Figure 6.3), especially in the plasma core. 


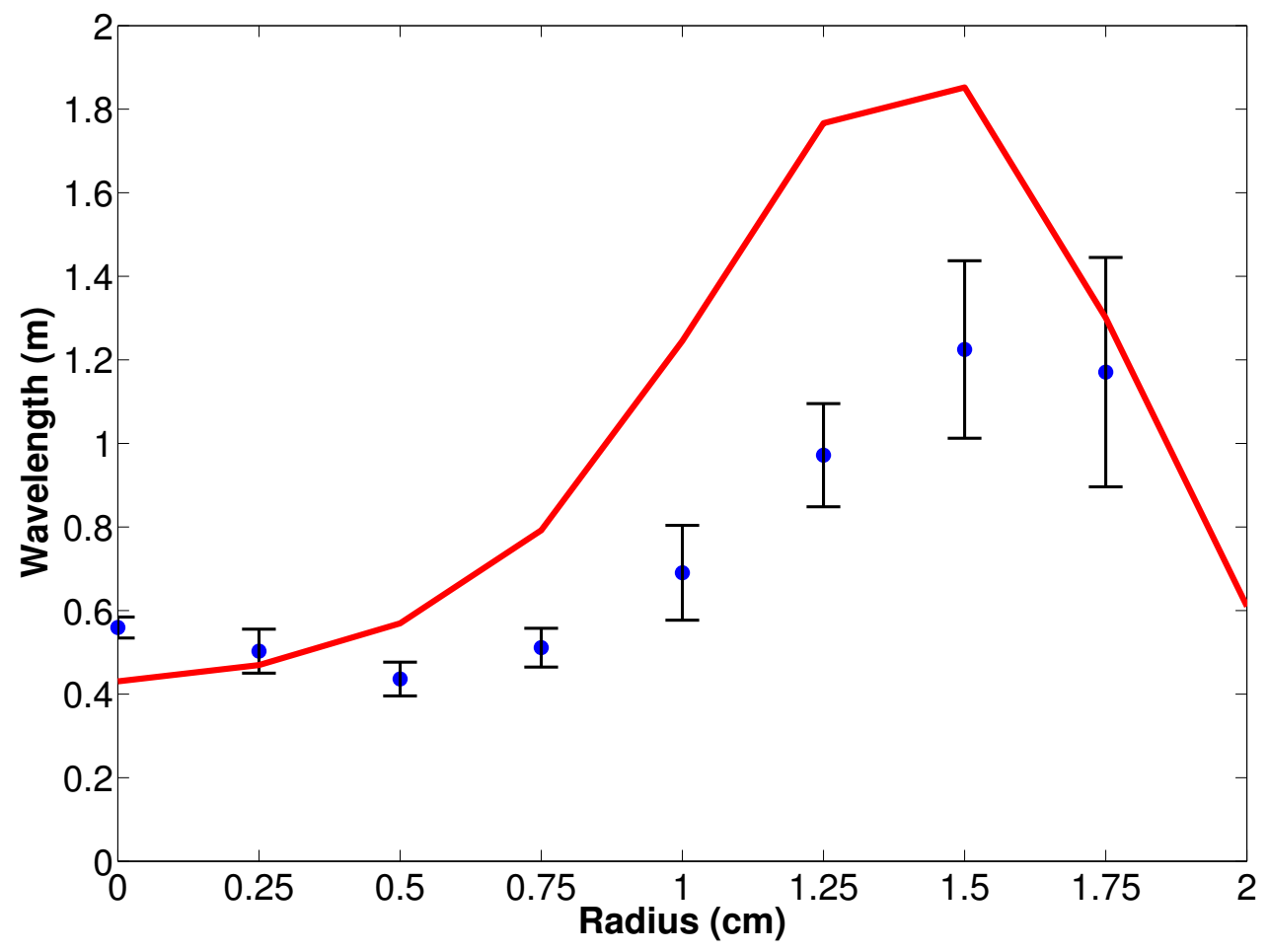

Figure 6.3: Alfvén wavelength measurements in HELIX compared to the theoretical predicted wavelength based on the density and gradient density scale measurements. The qualitative behavior of the measured wavelength is in good agreement with the predicted wavelength. 


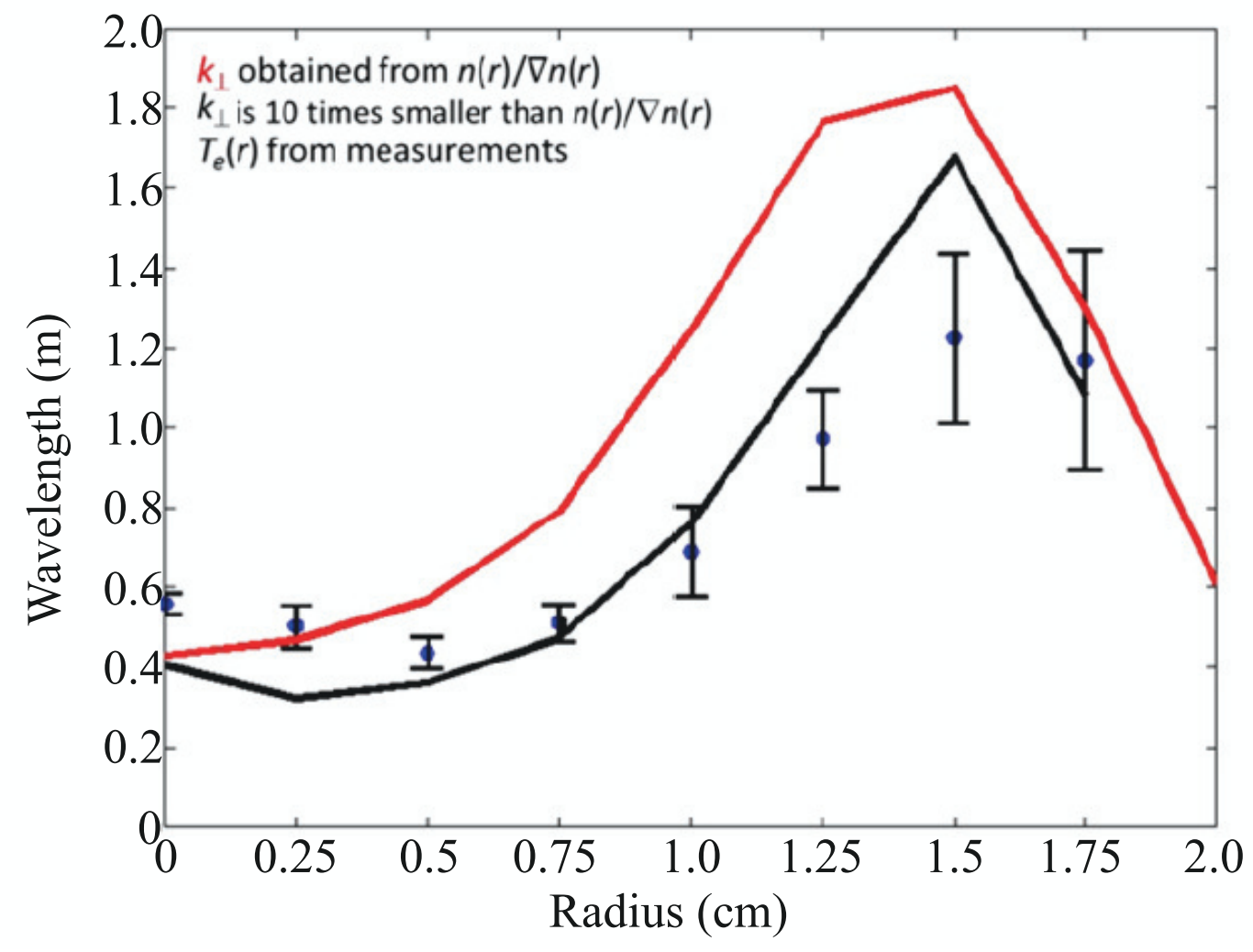

Figure 6.4: Alfvén wavelength measurements in HELIX compared to the theoretical predicted wavelength based on the density and gradient density scale measurements. The red curve is the prediction from the inertial Alfvén wave dispersion relation shown in Figure 6.3. The black curve is the full dispersion relation, including finite frequency effects, electron-ion collisions, electron Landau damping, and perpendicular wavelngth (ten times smaller than the gradient length scale; indicating that azimuthal circumference rather than radial scale may be the relevant dimension). The measured wavelength is in much better agreement with the predictions from the full dispersion relation. 


\subsection{Time Series Analysis Techniques}

To analyze a time-dependent signal appropriately, it is necessary to thoroughly understand the nature of that signal. A signal is deterministic if it is exactly predictable for the time span of interest while it is nondeterministic (stochastic or random) if it cannot be predicted exactly. Deterministic signals are well-described with mathematical models, while nondeterministic signals must be described with probabilities and statistical properties. Deterministic signals can be further broken down into two types: periodic and transient signals. Periodic signals repeat exactly at regular intervals (for example, a sine wave) while transient signals appear for only a short period of time but can be represented analytically during that time (for example, a damped harmonic oscillator). Nondeterministic signals can also be broken down into two types: stationary and non-stationary signals. A stationary signal has statistical properties that do not change with time while the statistical properties of a non-stationary signal do change with time. While these categories are useful, it is important to keep in mind that signal classifications are not rigid. No real measured signal is ever completely deterministic and may have several of the characteristics discussed above. These general classification guidelines are still useful when considering the most appropriate signal 
processing methods.

One way to visualize the complex information in a time signal is to think about it like a piece of music. ${ }^{[64]}$ If a musical score represents a time series, each note in that score carries four pieces of information: the vertical position (frequency), the horizontal location (the time), the type of note and tempo (duration), and the accent (intensity). Having only one piece of information about the score (such as knowing what frequencies occur within it but not when, or with what duration and intensity) makes the music unrecognizable. Additionally, many pieces of music have the same global information (key signatures, such as "C major"), which makes identifying the music by this single global feature impossible. ${ }^{[65]}$ Time-resolved information (which frequencies happen at what time, how long those frequencies last) is necessary to identify a unique musical composition. Bringing the analogy back to analyzing experimental time signals, we can see that a frequency spectrum of a time series measurement does not fully describe that time series, especially if some of the signals embedded in the time series are transient.

Signal-processing techniques typically transform a signal in the time domain into another domain with the aim of extracting information otherwise not readily observable in the original time-domain form. These techniques 
generally compare a time series measurement to a template function in order to determine the level of "similarity" between the two functions. The inner product of the original signal with a template function quantifies the degree of similarity. Mathematically in the Lebesgue space $L^{2}(\mathbb{R})$, an inner product between the two functions is written as

$$
\langle p, q\rangle \equiv \int_{-\infty}^{\infty} p(t) q^{*}(t) \mathrm{d} t
$$

where ()$^{*}$ denotes the complex conjugate and $\mathbb{R}$ is the real number line. ${ }^{[66]}$ Typically, the comparison is made between a time-domain signal $x(t)$ and a set of template functions $\left\{\psi_{n}(t)\right\}_{n \in \mathbb{Z}}$ where $\mathbb{Z}$ is the set of integers. The inner product of the time-domain signal and the template functions yields a set of integers that can be expressed using Eqn. 6.6 as either ${ }^{[67]}$

$$
c_{k}=\int_{-\infty}^{\infty} x(t) \psi_{k}^{*}(t) \mathrm{d} t
$$

or, more generally,

$$
c_{k}=\left\langle x, \psi_{k}\right\rangle \text {. }
$$


Similar to the scalar product of two vectors, the inner product of $x(t)$ with $\left\{\psi_{n}(t)\right\}_{n \in \mathbb{Z}}$ is maximized when the two functions are most similar. Both the Fourier transform and the wavelet transform take advantage of inner products to quantify the amount of similarity between the time series being analyzed and the template functions being used for comparison. The short time Fourier transform (STFT) method delivers coarse time and frequency information for a single time series. The wavelet transform is an adaptive time-frequency analysis method that optimizes time-resolution information for each individual frequency embedded in the time series.

\subsubsection{The Fourier Transform}

One of the most widely-used signal processing tools in science and engineering is the Fourier transform. The Fourier transform operates on a continuous time series $x(t)$ that is in the time domain and yields the frequency composition of it by transforming it into the frequency domain. The French mathematician Jean Baptiste Joseph Fourier is credited with discovering the principles behind this technique in 1807. Due to objections from contemporaries such as Joseph-Louis Lagrange, Fourier did not publish his findings until 1822 in The Analytic Theory of Heat. Using the inner product 
form from Eqn. 6.6, the Fourier transform is written as

$$
X(\nu)=\left\langle x, e^{i 2 \pi \nu t}\right\rangle=\int_{-\infty}^{\infty} x(t) e^{-i 2 \pi \nu t} \mathrm{~d} t
$$

where $\nu$ is the frequency of a sinusoidal template function. It is important to note that Eqn. 6.9 assumes that the signal has finite energy $\varepsilon$. The energy of signal $x(t)$ is defined as the integral of the squares of all values of the signal. For an inverse Fourier transform to exist, the energy of the signal must converge ${ }^{[68]}$

$$
\varepsilon=\int_{-\infty}^{\infty}|x(t)|^{2} \mathrm{~d} t<\infty
$$

The term "energy" is used here because it is a common occurrence in physics that the sum of the squares is used when various types of energy are calculated. ${ }^{[69]}$ To take the signal from the frequency domain to the time domain, the inverse Fourier transform is used.

$$
X(t)=\int_{-\infty}^{\infty} x(\nu) e^{i 2 \pi \nu t} \mathrm{~d} \nu
$$


For Eqn. 6.9 to be useful, knowledge of the complete time history of the signal from $-\infty$ to $\infty$ is necessary. Conversely, Eqn. 6.11 says that every value of $x(t)$ at a single instant, $t$ can be written as an infinite superposition of complex exponentials, infinite non-local waves. This means that the Fourier transform is very well suited to analysis of deterministic, periodic signals, but not transient or nonstationary signals.

Equations 6.9 and 6.11 are intended for application on continuous signals. Real signals, however, are acquired through discrete sampling over a finite time interval at discrete time intervals, $\Delta T$, over a total measurement time span $T$. The analysis of discrete signals is accomplished with the discrete Fourier transform (DFT)

$$
\operatorname{DFT}\left\{x_{n}\right\}=\hat{x}_{k}=\frac{1}{N} \sum_{n=0}^{N-1} x_{n} e^{-i 2 \pi \nu_{k} n \Delta T}
$$

where $N=T / \Delta T$ is the total number of samples, $n$ is the sample index, $x_{n}$ is the $n^{t} h$ sample of a discretely sampled physical quantity, and $\nu_{k}=k / T, k=$ $0,1,2, \ldots \ldots,(N-1)$ are the discrete frequency components of the physical 
quantity being sampled. The inverse DFT is ${ }^{[67]}$

$$
D F T^{-1}\left\{\hat{x}_{k}\right\}=x_{n}=\frac{1}{\Delta T} \sum_{\nu_{k}=0}^{(N-1) / T} \hat{x}_{k} e^{i 2 \pi \nu_{k} n \Delta T} .
$$

Calculating the DFT of a real measured signal takes a lot of computational power. The DFT of $N$ samples requires the multiplication of a $N \times N$ matrix and the calculation steps on the order of $N^{2}$. This means that improving the time resolution of a measurement considerably increases the computational costs of Fourier analyzing that measurement. Until the Cooley-Tukey algorithm provided a computationally efficient way to implement DFT on real signals, DFT analysis in science and engineering was rare. The CooleyTukey algorithm is a variant of what is now called the Fast Fourier transform (FFT). Alternate versions of the FFT were known as far back as 1805 (when Carl Friedrich Gauss authored a version that predated Fourier's work by approximately two years). ${ }^{[70]}$ The Cooley-Tukey FFT (simply called the FFT) has become the dominant FFT technique because it requires fewer operations than other methods, reducing the number of calculations to $N \log (N)$. The FFT is a recursive algorithm that is easy to computationally implement. 
The lowest frequency that can be resolved with an FFT, $\Delta \nu$, is given by

$$
\Delta \nu=\frac{\nu_{s}}{N}=\frac{1}{T}
$$

where $\nu_{s}$ is the sampling frequency. As can be seen from Equation 6.14, the frequency resolution is determined by the acquisition time.

Just as with the continuous Fourier transform, the amplitude of the complex coefficients for each frequency component quantify how "much" of a given frequency is embedded in the measured signal. The unnormalized power spectrum

$$
S_{x_{n} x_{n}}\left(\omega_{k}\right)=F F T\left\{x_{n}\right\} * F F T^{*}\left\{x_{n}\right\}
$$

is the amplitude of each discrete frequency component written in a compact mathematical form. While Fourier analysis isolates individual frequency components in a time series, the time-behavior of those components is lost. One of the most powerful methods for analyzing time-frequency behavior of measured signals is the wavelet transform. 


\subsubsection{The Wavelet Transform}

At its most basic level, wavelet analysis is a mathematical tool for understanding both the dominant modes (frequencies) within a time series and the way those modes change with time. Wavelet transforms use local base functions that are stretched and translated with a flexible resolution in both frequency and time to provide information on the time-frequency behavior of a measured physical signal. The first published account of what are now called wavelets was in Alfred Haar's 1909 dissertation. While Haar created a set of rectangular basis functions, the squeezing and stretching of those basis functions, as well as the term "wavelet" did not appear until the work of Jean Morlet and Alex Grossman, along with a team of other researchers at the Marseille Theoretical Physics Center in France, in the 1970's. The single biggest advance in the use and study of wavelets came in 1988 when Yves Meyer and Stéphane Mallat developed multi-resolution analysis (MRA) for wavelet transforms. MRA combined wavelet theory with fast discrete signal processing techniques and provided a mathematical framework for creating new base wavelets tailored to specific research needs. Ingrid Daubechies and other scientists added new families of wavelet transforms and to the field. ${ }^{[71]}$ This work set in motion a proliferation of wavelet analysis into such varied 
disciplines as astronomy, biology, medicine, ${ }^{[71]}$ image and audio processing (compression, detection, and denoising), ${ }^{[69]}$ manufacturing, ${ }^{[67]}$ and climate observations. ${ }^{[64],[72],[73]}$ Van Milligen et al. ${ }^{[74]}$ were the first to publish wavelet analysis of laboratory plasma investigations in 1995. The wavelet transforms continue to be employed for analyzing intermittent phenomena in plasma physics to this day. ${ }^{[75],[76],[77]}$

A continuous wavelet transform, $W$, is expressed as an inner product, similar to a Fourier transform, using Equations 6.7 and 6.8

$$
W_{(S, \tau)}=\left\langle x,_{S, \tau}\right\rangle=\int_{-\infty}^{\infty} x(t) \psi_{S, \tau}^{*}\left(\frac{t-\tau}{S}\right) \mathrm{d} t
$$

where $\tau$ is the translation position, $S$ is the scale parameter which controls the dilation, and $\psi_{S, \tau}$ is the "daughter wavelet" or just the "wavelet" which is derived from

$$
\psi_{(S, \tau)}\left(\frac{t-\tau}{S}\right)=\frac{1}{\sqrt{S}} \psi\left(\frac{t-\tau}{S}\right), s>0, \tau \in \mathbb{R}
$$

where $\psi$ is the "mother wavelet," the "analyzing wavelet," or the "wavelet 

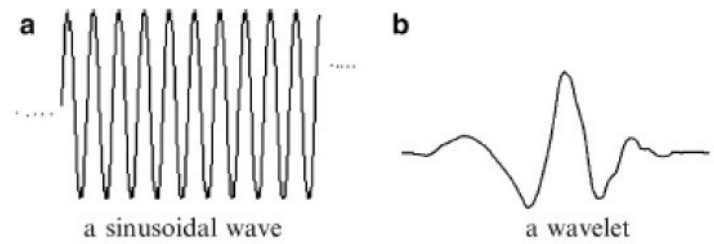

Figure 6.5: Representation of (a) a sine wave and (b) a Daubechies wavelet. Figure obtained from Gao and Yan. ${ }^{[67]}$

function." Substituting Eqn. 6.17 in Eqn. 6.16 yields

$$
W_{(S, \tau)}=\frac{1}{\sqrt{S}} \int_{-\infty}^{\infty} x(t) \psi_{S, \tau}^{*}\left(\frac{t-\tau}{S}\right) \mathrm{d} t
$$

The scale parameter $S$ depends on the properties of the mother wavelet and is inversely proportional to the local frequency of the signal. ${ }^{[78],[64]}$

A wavelet is a waveform with a finite duration and a mean value of zero. These two requirements are imposed by the admissibility condition which ensures that mother wavelets are well localized and oscillate. ${ }^{[79]}$ A visual comparison between a wavelet function and a sine wave is provided in Figure 6.5 .

The $1 / \sqrt{S}$ normalizing factor in Equation 6.18 forces all wavelets to have the same unit energy as the dilation process is applied. This constant unit energy allows the wavelet transforms to be comparable at each scale param- 


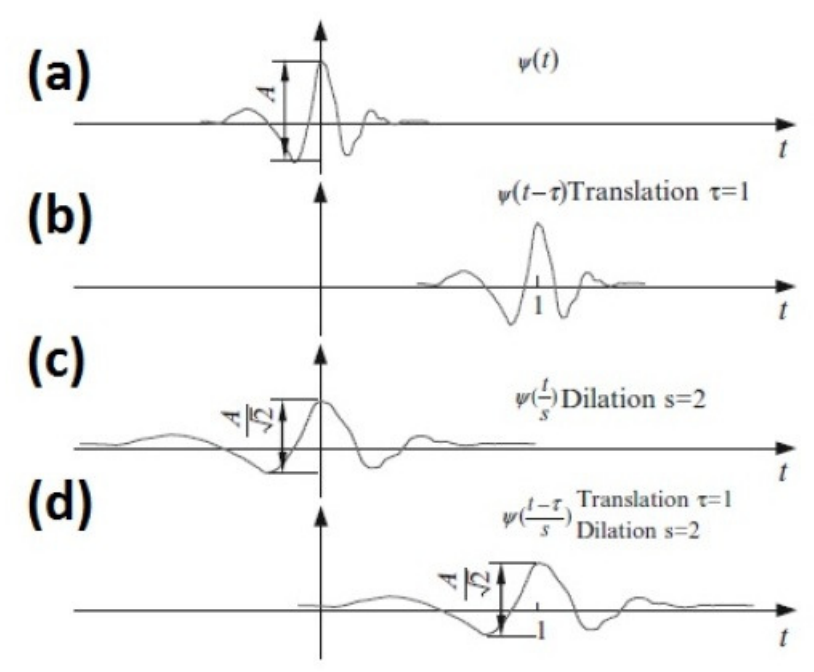

Figure 6.6: Wavelet translation by the time constant $\tau$ and dilation by the scaling factor $S$ while constant energy is maintained. In (a) the original mother wavelet is shown centered at $t=0$, untranslated and undilated. In (b) the mother wavelet is shown translated by $\tau=1$. In (c) the mother wavelet is shown dilated by the scaling factor $S=2$. In (d) the mother wavelet is shown translated by $\tau=1$ and dilated by $S=2$. Figure adapted from Gao and Yan. ${ }^{[67]}$

eter. An illustration of the translation and dilation processes outlined in Equation 6.18 is shown in Figure 6.6.

In her book Ten Lectures on Wavelets, Ingrid Daubechies outlines two classes of wavelet transforms: the continuous wavelet transform and the discrete wavelet transform. ${ }^{[0]}$ Discrete wavelet transforms are subdivided into two categories: orthonormal and non-orthonormal. Orthonormal discrete wavelet transforms used in MRA are better suited for data compression and 
reconstruction because of the efficiency of storing signal energy that they provide. Continuous wavelet transforms are not orthonormal and therefore are not suited as well for compression, but they do vary $S$ and $\tau$ continuously, unlike discrete wavelet transforms. This makes continuous wavelet transforms better suited for analyzing smooth variations in a measured time series. Continuous wavelet transforms are used in the analysis of the data in this work.

\subsubsection{Wavelet Analysis of Magnetic Sense Coil Probe Signals}

When a properly chosen wavelet is used to analyze the time series of a signal, it shows how the frequency components in that signal change over time. Figures 6.6-6.11 show the results of wavelet analysis of the time series measurements taken with the magnetic sense coil probe across the plasma column. Note the clean appearance of the excited Alfvén waves when the antenna turns on at $32.5 \mathrm{~ms}$. In the center of the plasma $(r=0 \mathrm{~cm})$ and in the strong density gradient, a continuous spread of frequencies between the perturbation frequency $(25 \mathrm{kHz})$ and the ion cyclotron frequency (30 $\mathrm{kHz}$ ) appears during the perturbation pulse. This spread of frequencies is consistent with the creation of a spread of parallel wavelengths; exactly what 
is expected for a turbulent cascade. Since $\lambda$ is $\sim 1 / f$ for Alfvén waves, a spread in frequencies from the driving frequency to higher frequencies is a cascade to higher $k_{\|}$, shorter $\lambda$, waves.

While the intensity color plots are useful for understanding the timefrequency behavior of the magnetic sense probe signals, and showing that the spread of frequency modes happens only when the perturbation pulse is present, discerning the color differences that show different intensities at different frequencies is difficult. Another way to visualize intensity versus frequency information is to sum the signal amplitude of each frequency value over time and plot the resulting total signal amplitude as a function of frequency. This yields a two-dimensional plot of signal amplitude versus frequency which, although integrated over the entire perturbation pulse, shows whether there is signal at a single, sharply-defined frequency or over a continuous spread of frequencies. If the signal amplitude rises above the background noise only at a single well-defined frequency value (such as the 25 $\mathrm{kHz}$ perturbation frequency), only that frequency mode is propagating in the plasma. If, instead, the signal rises above the background noise for a continuous range of frequencies, it shows the propagation of multiple waves.

In all of the wavelet analysis plots shown here there are discrete structures 
at $17 \mathrm{kHz}, 24 \mathrm{kHz}$, and $34 \mathrm{kHz}$. These are likely drift waves, which have been observed in helicon plasmas before. ${ }^{[81],[82],[83]}$ Drift waves arise from density gradients, so it is expected we would observe them during these experiments given the strong density gradient across the plasma column. The peak at 34 $\mathrm{kHz}$ is a harmonic of the $17 \mathrm{kHz}$ signal and is larger in amplitude because the magnetic sense coil sensitivity scales directly with frequency. The turbulence during the perturbation pulse could arise from non-linear interaction between the driven $25 \mathrm{kHz}$ Alfvén wave and the $24 \mathrm{kHz}$ drift wave.

Figure 6.6(a) shows the intensity of frequency modes between 0 and 50 $\mathrm{kHz}$ of the magnetic sense coil probe signal at $r=0 \mathrm{~cm}$. In this plot it easy to see that a continuous band of frequency modes appears between $25 \mathrm{kHz}$ (the driven perturbation frequency) and $30 \mathrm{kHz}$ (the ion cyclotron frequency) during the perturbation pulse. Figure 6.6(b) shows the total signal amplitude at each frequency value (the sum of the intensity values for each frequency over the entire timespan of 6.6(a)). This amplitude versus frequency plot shows the spread of wave modes even more clearly. This continuous spread of frequency (wavelength) modes indicates turbulence in the plasma at $r=0$ $\mathrm{cm}$.

Figure 6.7 shows plasma turbulence at $r=0.25 \mathrm{~cm}$, but with much less 
power in the turbulent modes than seen at $r=0 \mathrm{~cm}$. At $r=0.5 \mathrm{~cm}$ (shown in Figure 6.5), the turbulence disappears all together. Figures 6.9-6.11 show that the turbulent spread reappears at larger radii, but never as strongly as at $r=0 \mathrm{~cm}$. More sensitive magnetic sense coil probes are needed to better measure weaker signals, and an array of such probes at multiple axial locations is necessary to better characterize the turbulent spectrum of the wave perturbation.

A helpful way to visualize the change in the turbulent spectrum across the plasma column is to plot the area under the curve between $25 \mathrm{kHz}$ (the perturbation frequency) and $30 \mathrm{kHz}$ (the ion-cyclotron frequency) in the signal amplitude versus frequency plots for Figures 6.6-6.11. This area under the curve is a measure of the total power in the turbulent spectrum at each radial location. The change in the turbulent spectrum across the plasma column is shown in Figure 6.13 


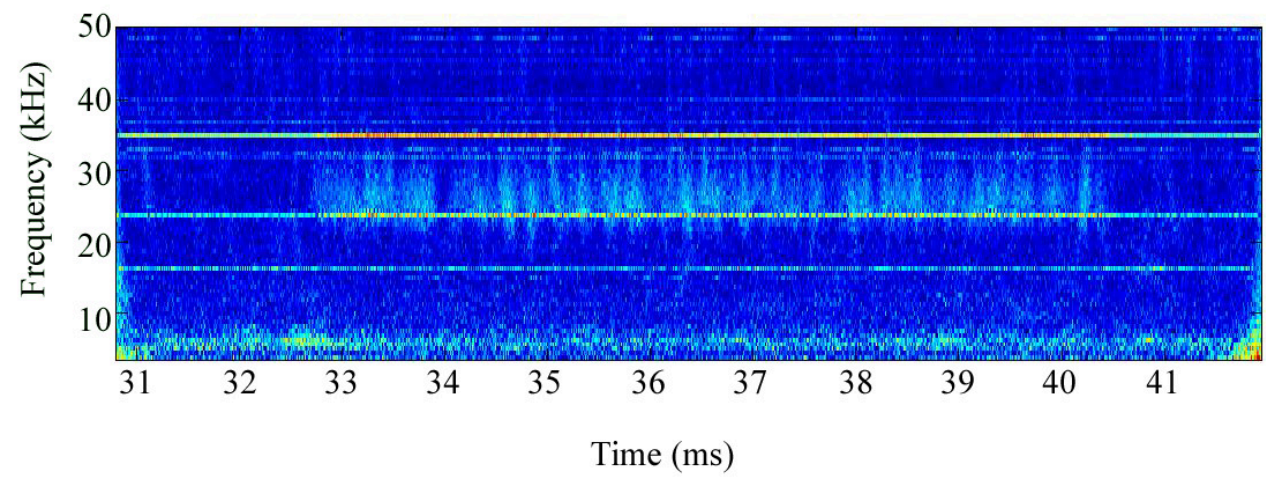

(a)

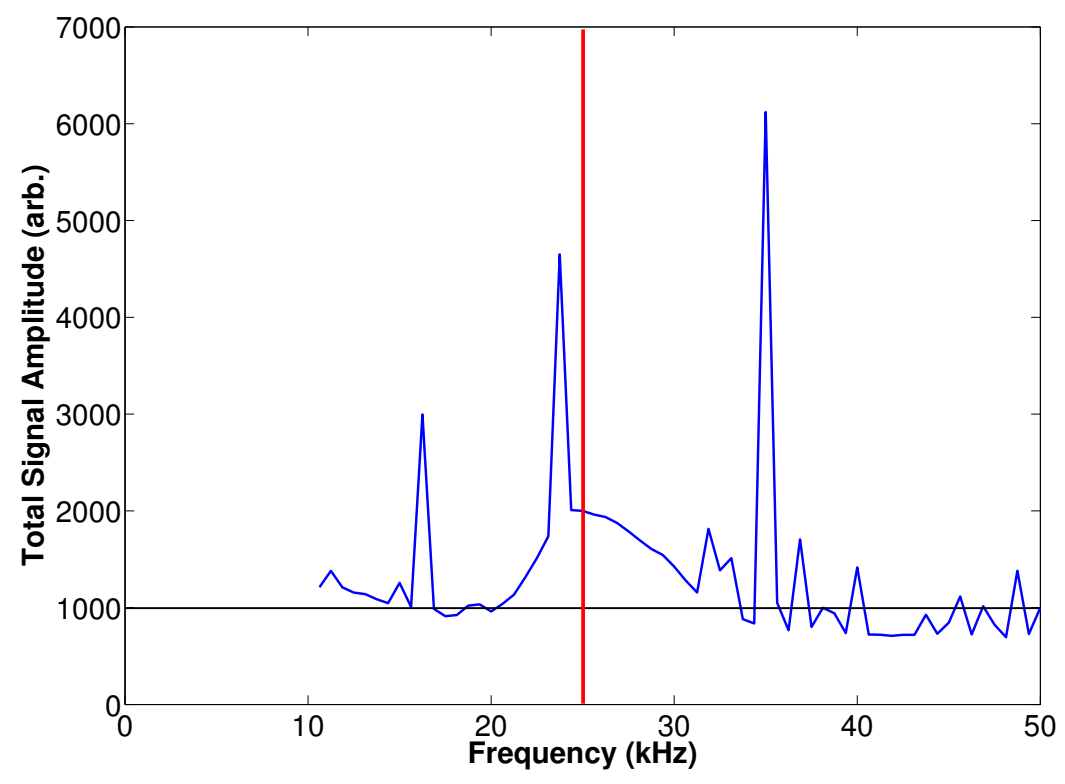

(b)

Figure 6.7: (a)Wavelet analysis of the magnetic sense coil probe time series at $r=0 \mathrm{~cm}$. A spread of frequency modes between $25 \mathrm{kHz}$ and $30 \mathrm{kHz}$ appears only during the perturbation pulse $(32.5 \mathrm{~ms}-40.5 \mathrm{~ms})$. (b)The intensity versus frequency summation shows a continuous spread of frequency modes above the background noise between $25 \mathrm{kHz}$ and $30 \mathrm{kHz}$. This intensity spread across a continuous range of frequency modes indicates turbulence. The vertical red line marks the perturbation frequency, while the sharp peaks at $17 \mathrm{kHz}, 24 \mathrm{kHz}$, and $34 \mathrm{kHz}$, are due to drift waves. 


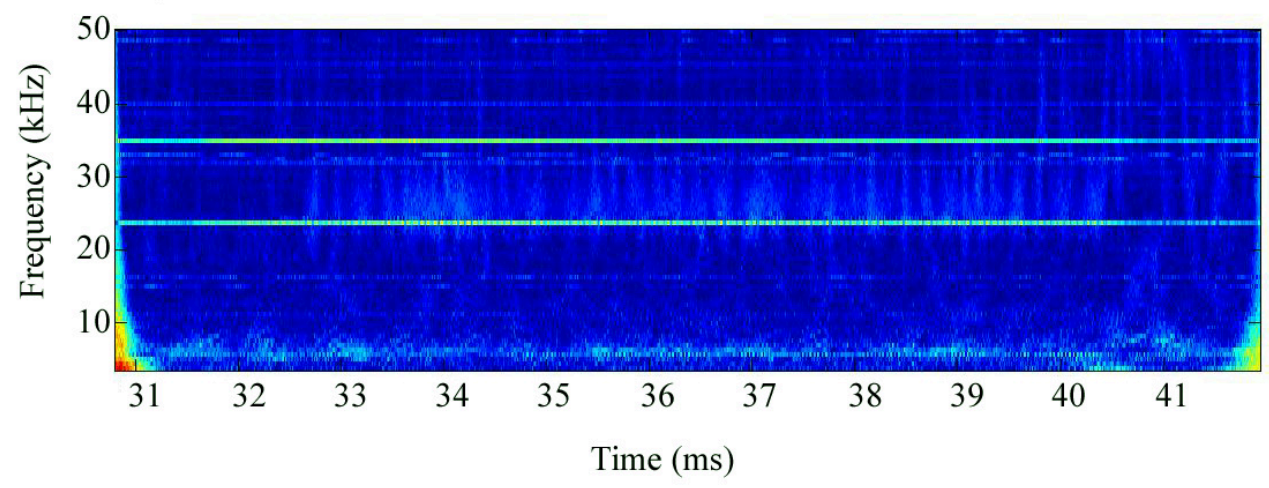

(a)

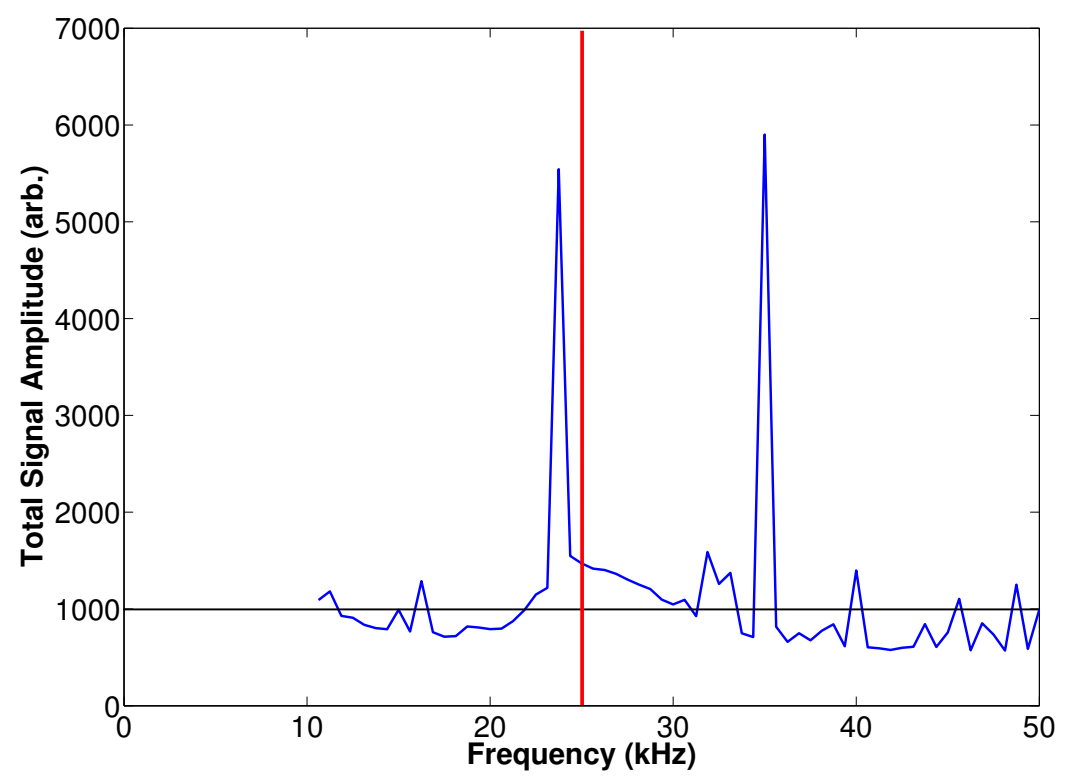

(b)

Figure 6.8: (a)Wavelet analysis of the magnetic sense coil probe time series at $r=0.25 \mathrm{~cm}$. The spread of frequency modes is still present during the perturbation pulse, but the amplitude (power) of those modes is weaker than at $r=0 \mathrm{~cm}$. (b)The intensity versus frequency summation shows less wave power in the turbulent modes than at $r=0 \mathrm{~cm}$. The vertical red line marks the perturbation frequency, while the sharp peaks at $17 \mathrm{kHz}, 24 \mathrm{kHz}$, and 34 $\mathrm{kHz}$, are due to drift waves. 


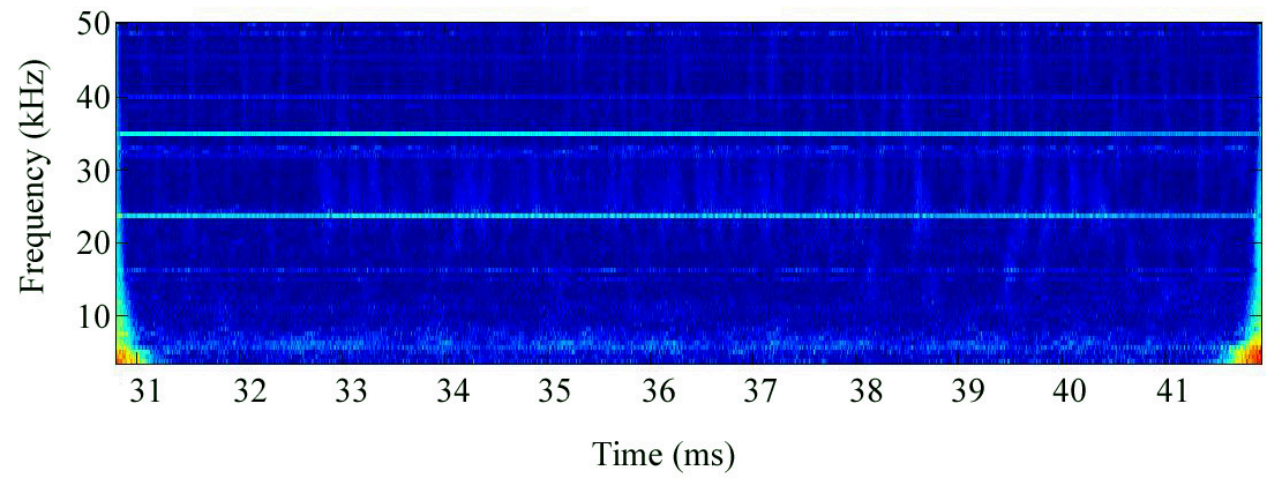

(a)

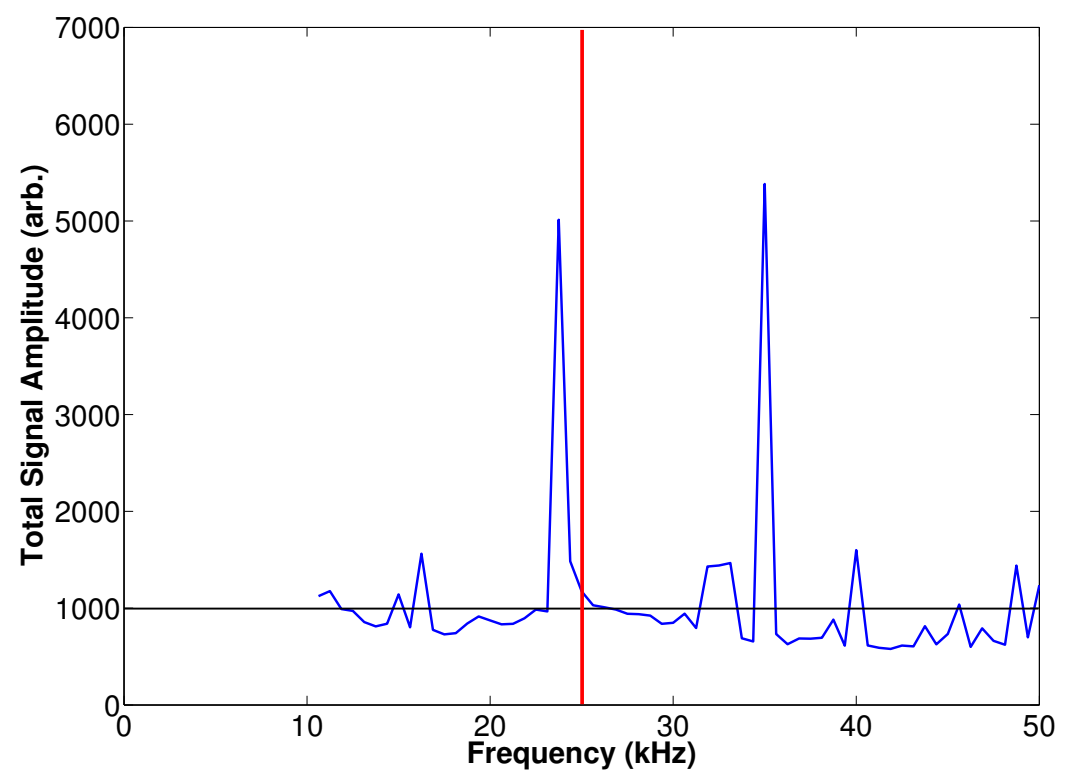

(b)

Figure 6.9: (a)Wavelet analysis of the magnetic sense coil probe time series at $r=0.50 \mathrm{~cm}$. (b)The intensity versus frequency summation shows no turbulence present at $r=0.50 \mathrm{~cm}$. The vertical red line marks the perturbation frequency, while the sharp peaks at $17 \mathrm{kHz}, 24 \mathrm{kHz}$, and $34 \mathrm{kHz}$, are signature of drift wave modes. 


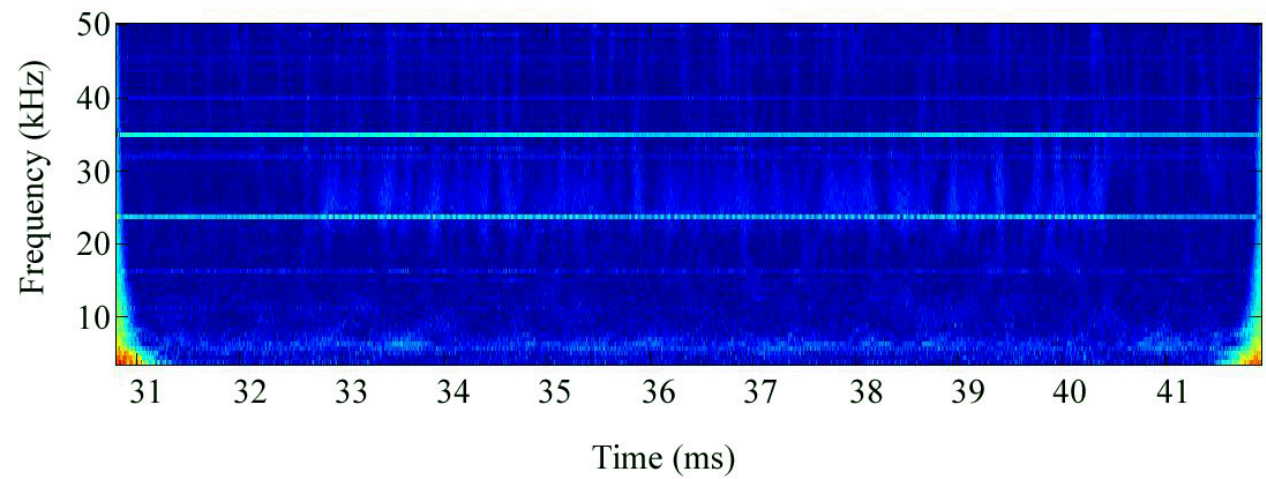

(a)

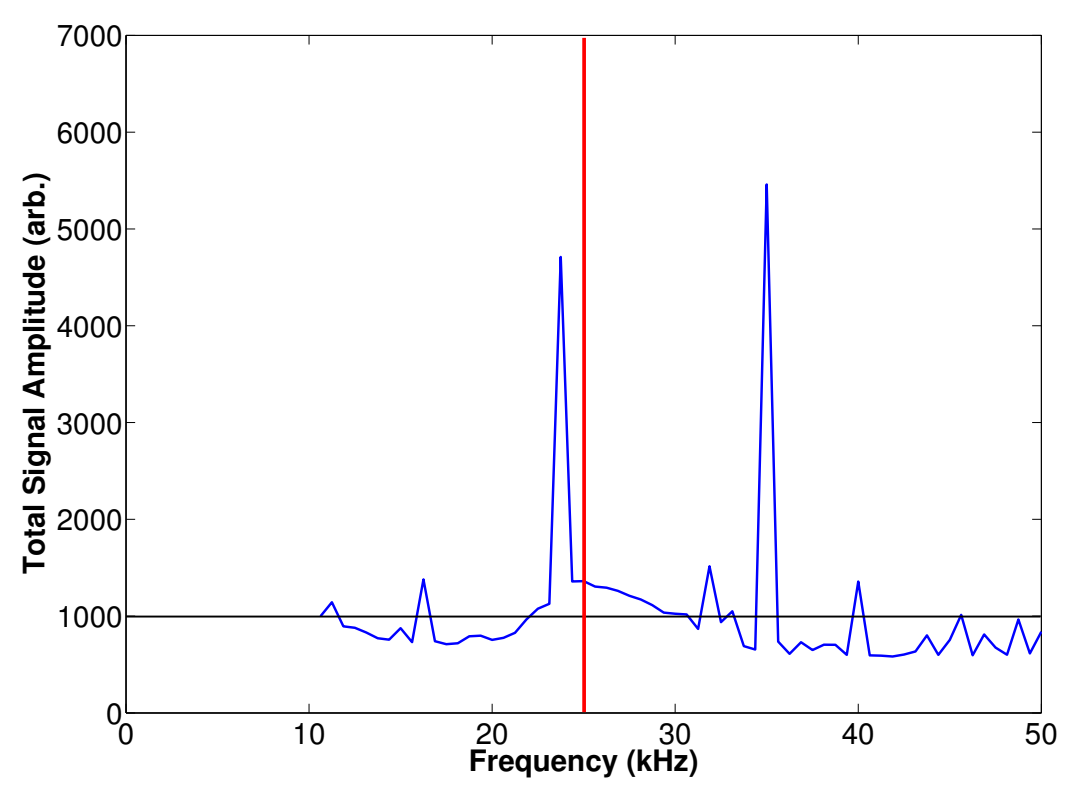

(b)

Figure 6.10: (a)Wavelet analysis of the magnetic sense coil probe time series at $r=0.75 \mathrm{~cm}$. (b)The intensity versus frequency summation shows turbulence at this location similar to that seen at $r=0.25 \mathrm{~cm}$. The vertical red line marks the perturbation frequency, while the sharp peaks at $17 \mathrm{kHz}, 24 \mathrm{kHz}$, and $34 \mathrm{kHz}$, are due to drift waves caused by the density gradient. 


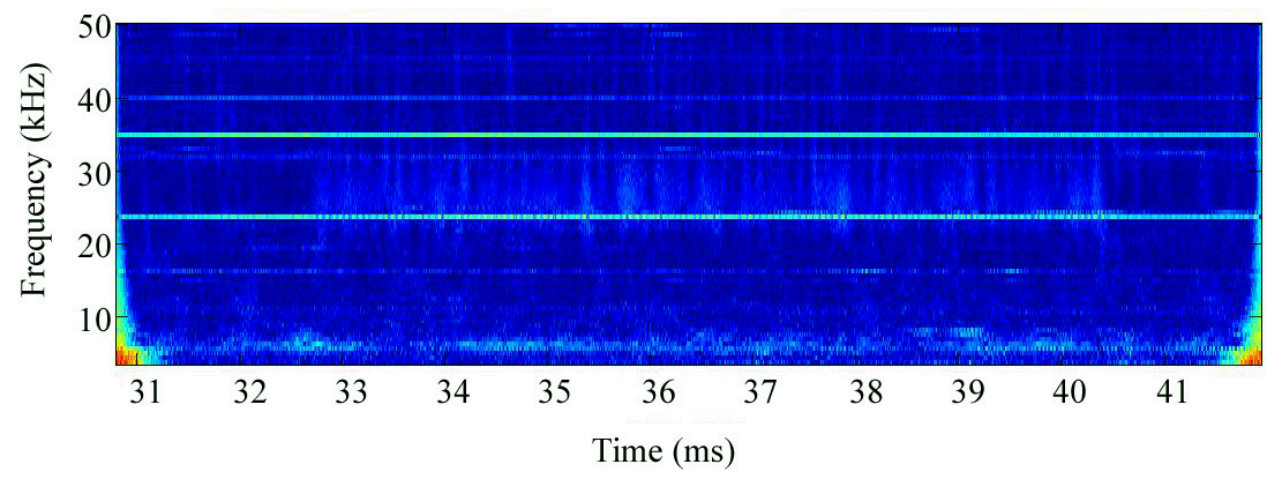

(a)

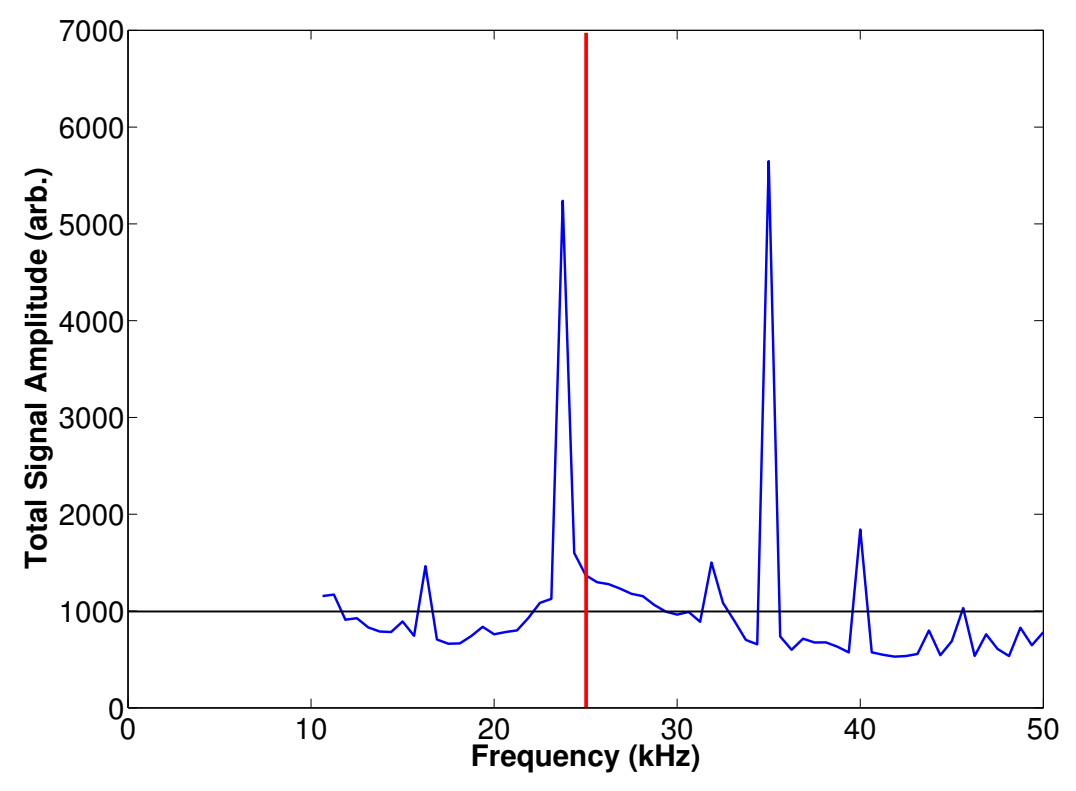

(b)

Figure 6.11: (a)Wavelet analysis of the magnetic sense coil probe time series at $r=1.0 \mathrm{~cm}$. (b)The intensity versus frequency summation shows turbulence in the plasma, and the wave power in the turbulent modes at this location is comparable to that at $r=0.25 \mathrm{~cm}$ and $r=0.75 \mathrm{~cm}$. The vertical red line marks the perturbation frequency, while the sharp peaks at $17 \mathrm{kHz}, 24 \mathrm{kHz}$, and $34 \mathrm{kHz}$, are due to drift waves. 


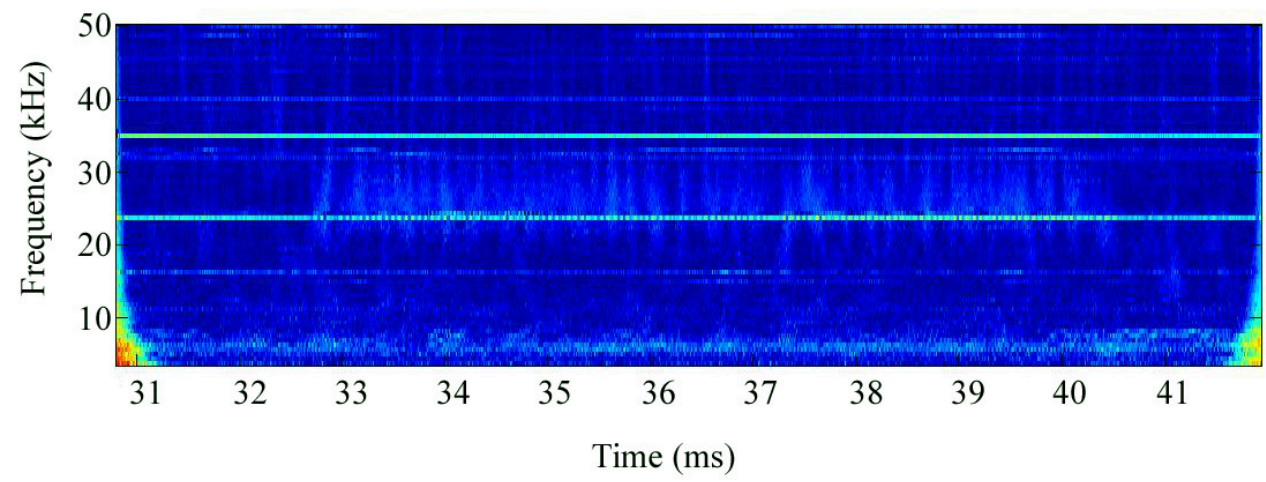

(a)

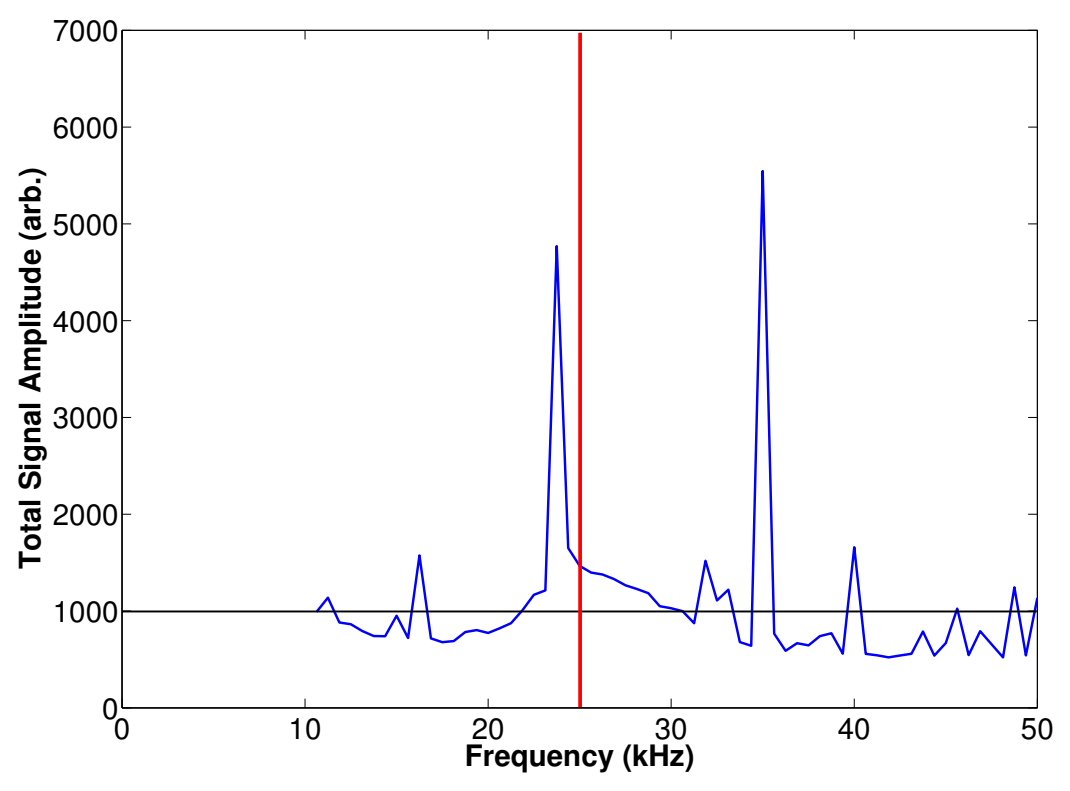

(b)

Figure 6.12: (a)Wavelet analysis of the magnetic sense coil probe time series at $r=1.25 \mathrm{~cm}$. (b)The intensity versus frequency summation shows turbulence in the plasma, and the wave power in the turbulent modes at this location is comparable to that at $r=0.25 \mathrm{~cm}$, as well as $r=0.75 \mathrm{~cm}$ and $r=1.00$ $\mathrm{cm}$. The vertical red line marks the perturbation frequency, while the sharp peaks at $17 \mathrm{kHz}, 24 \mathrm{kHz}$, and $34 \mathrm{kHz}$, are signatures of drift waves. 


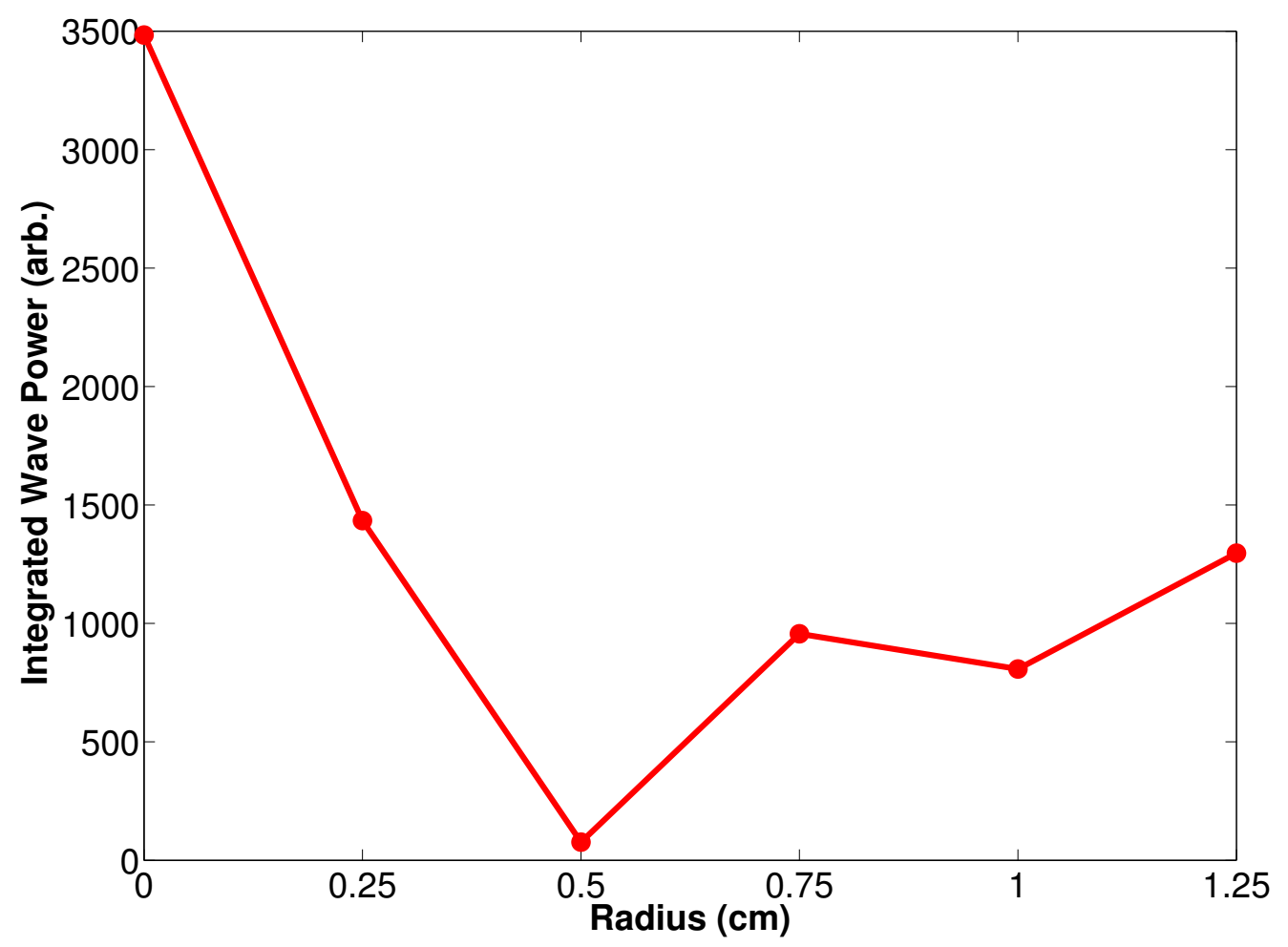

Figure 6.13: The area under the curve between $25 \mathrm{kHz}$ and $30 \mathrm{kHz}$ from the wavelet amplitude versus frequency plot for each radial location is shown. Note that turbulent power is large at $r=0 \mathrm{~cm}$, drops to nearly zero at $r=0.5$ $\mathrm{cm}$, and then returns at a low level for larger radii. Comparison of this plot with measurements of normalized ion heating across the plasma column (see Figure 7.17) indicate a correlation between the turbulence and ion heating observations. 


\section{Chapter 7}

\section{Time-Resolved Laser-Induced Fluorescence (LIF)}

\section{Measurements}

\subsection{Laser-Induced Fluorescence Background and Theory}

Laser-induced fluorescence (LIF) is a non-perturbative, spatially-resolved and temporally-resolved diagnostic technique for measuring particle velocity distribution functions. In an LIF system, a narrow bandwidth laser is tuned to a natural absorption line of an ion or atom. Absorption of photons by the ions or atoms pumps them into an excited state and the decay emission back to a relaxed state is measured in order to determine the velocity distribution function (VDF) of the ions or atoms being investigated. By using injection and collection paths for the laser light and emitted light that cross in only one spatial location, LIF measurements can be localized to a specific location in the plasma; spatial resolutions of $\sim 1 \mathrm{~mm}^{3}$ are easily accomplished. Decay of the excited particles to the same initial state is called resonant LIF; decay to a third state, different from the initial state, is called non-resonant LIF. 


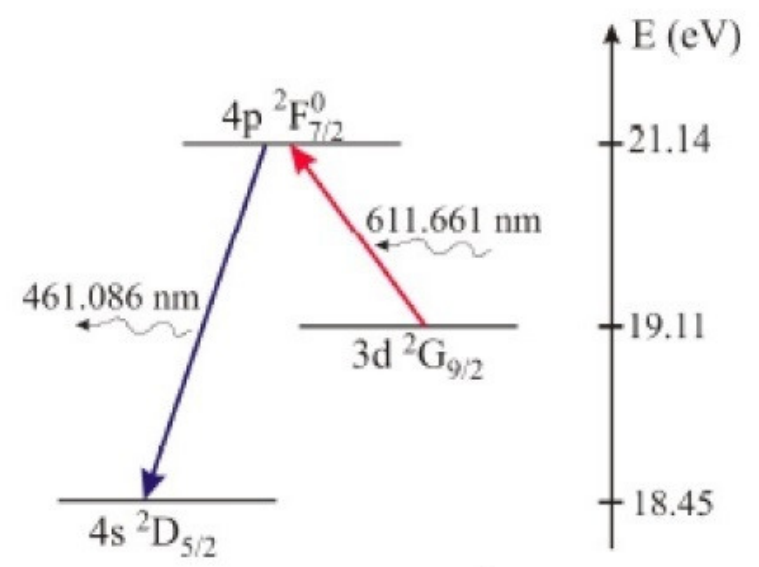

Figure 7.1: LIF scheme for Argon II.

Non-resonant LIF was used in this work to measure the VDF of Argon II metastable ions in HELIX (see Fig. 7.1).

In 1966 the first observation of LIF from molecules other than the lasing medium was made by Yardley and Moore. ${ }^{[84]}$ The first experimental observation of LIF in a plasma was made in 1975 by Stern and Johnson. ${ }^{[85]}$ The first use of a tunable dye laser for LIF was in 1979 by Meng and Kunze, ${ }^{[86]}$ while the frst velocity selective LIF measurements with a tunable dye laser in plasma were made by Hill et al. ${ }^{[87]}$ in 1983 . The techniques developed by Hill et al. are the basis for the LIF measurements made in this work. A comprehensive discussion of line-broadening mechanisms relevant to LIF in WVU-HELIX can be found in Keesee ${ }^{[88]}$ and Boivin. ${ }^{[89]}$ 
The width of an absorption line depends on several possible broadening mechanisms. Typical broadening mechanisms that must be considered include the natural line width, power broadening, Stark broadening, Zeeman broadening, and Doppler broadening. Zeeman broadening and Doppler broadening are the two dominant broadening mechanisms in the HELIXLEIA system. For the parameters of this work, all other possible broadening mechanisms are negligible. The Doppler broadening of the absorption line is directly related to the VDF of the ions. ${ }^{[89]}$

As the frequency of the narrow line-width laser is swept across the natural absorption line, moving ions or atoms absorb the Doppler shifted laser light in their own rest frame. Ions or atoms moving toward the laser absorb photons when the laser is tuned to a lower frequency than the natural absorption line, while ions or atoms moving away from the laser become excited when the laser is tuned to a frequency that is higher than the natural absorption line. Slower ions or atoms are excited at frequencies closer to the natural line (stationary ions or atoms absorb exactly at the natural absorption line), while faster ions or atoms are excited at frequencies farther from the natural line (a typical LIF trace is shown in Figure. 7.2).

By measuring the intensity of fluorescent emission from the excited state 


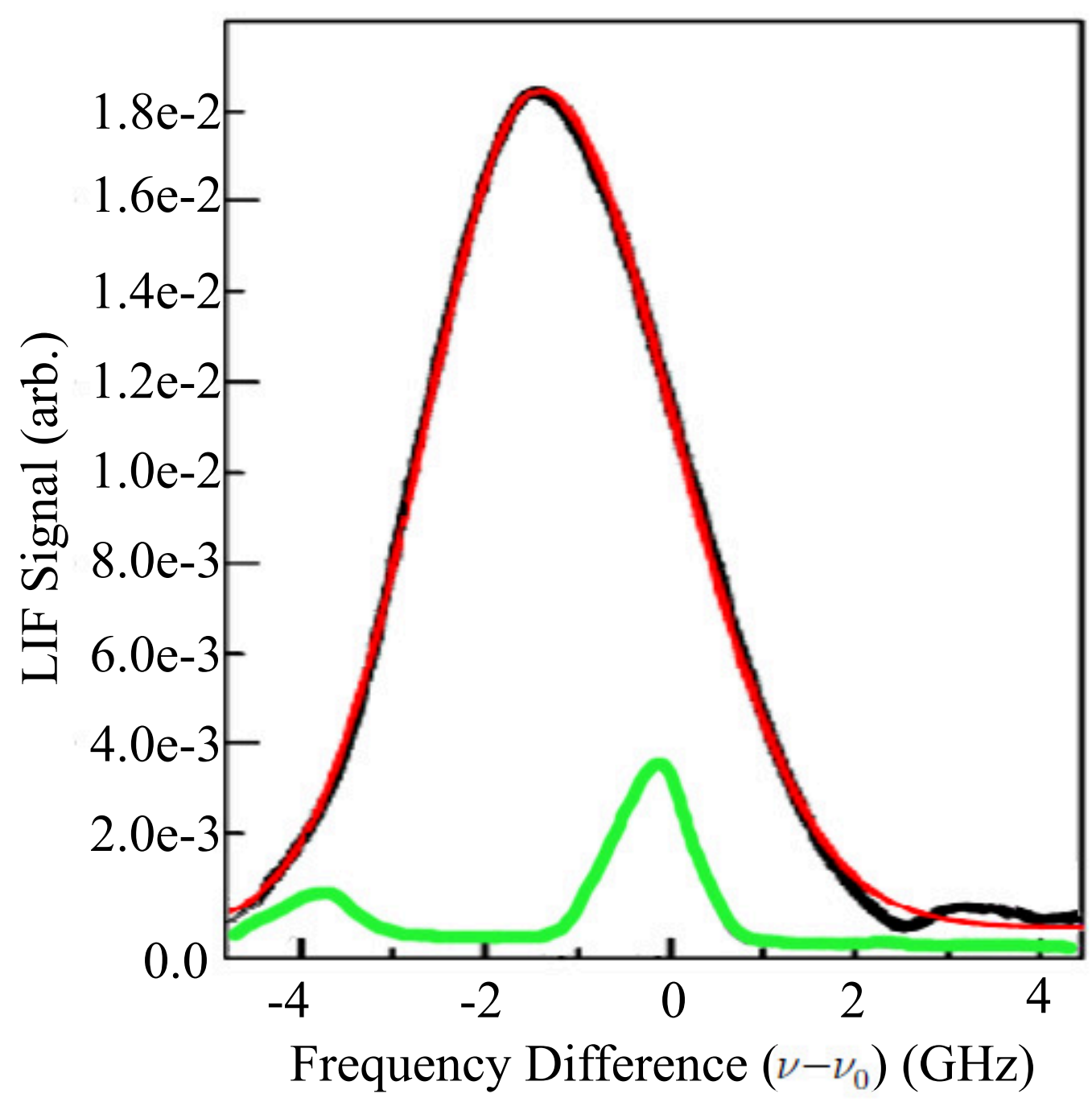

Figure 7.2: A typical argon plasma LIF trace, plotted as a function of frequency shift relative to the zero-velocity frequency of the iodine reference peak. The black line is the raw LIF signal, the red line is the Gaussian fit to the data, and the green line is the iodine reference signal. 
as a function of laser frequency a VDF is obtained. Analysis of the VDF measurement yields the ion or atom temperature, the density of the absorbing species (only if the LIF system is fully calibrated), and the net drift velocity. The Doppler broadening of an ion absorption line for a Maxwellian distribution is given by

$$
I(\nu)=I_{0} \exp \left(\frac{-m\left(\nu-\nu_{0}^{*}\right)^{2} c^{2}}{2 k_{B} T \nu_{0}^{2}}\right)
$$

where $I(\nu)$ is flux of absorbed photons as a function of frequency $\nu, I_{0}$ is the maximum flux of photon absorption, and $\nu_{0}^{*}=\nu_{0}+\nu_{0} v / c$ is the frequency of the transition when viewed in the laboratory rest frame.

Zeeman splitting yields linearly polarized $\pi$ lines $(\Delta m=0)$ and circularly polarized $\sigma$ lines $(\Delta m= \pm 1)$ for absorption between the initial and excited states in the presence of a strong magnetic field. Figure 7.3 illustrates the details of typical Zeeman splitting of the primary $611.6616 \mathrm{~nm}$ absorption line of argon. The $\pi$ lines are symmetric on either side of the transition with no magnetic field while the $\sigma$ lines are actually two sets of clustered lines, $\sigma+$ and $\sigma-$. The amplitude envelope of the individual $\sigma$ clusters is asymmetric, but the two clusters are symmetric with each other in respect to the zero 


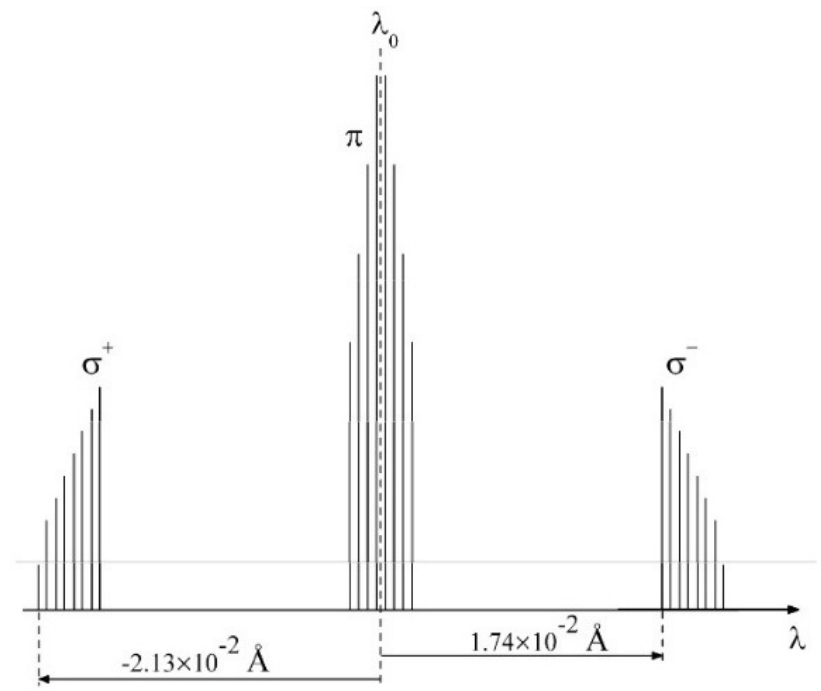

Figure 7.3: Illustration of the $\pi$ and $\sigma$ splitting for the $611.6616 \mathrm{~nm}$ argon II line. The height of each line corresponds to the statistical weight of each transition as a function of wavelength. Figure obtained from Sun. ${ }^{[90]}$

field transition line. The frequency shift between the $\sigma$ clusters and the zero field transition is linearly dependent on the magnetic field strength. The magnetic field strength at the measurement location can be determined from the shift of the $\sigma$ clusters.

The individual Zeeman components are also Doppler broadened in a thermal distribution of particles. For strong magnetic fields, accurate temperature measurements are possible only if the LIF signal is deconvolved into its separate Zeeman components. ${ }^{[91]}$ In the HELIX-LEIA system, the broadening due to Zeeman splitting of the $\pi$ lines and the two clusters of $\sigma$ lines is much smaller than the Doppler broadening of those same lines. ${ }^{[90]}$ This means that 
both clusters of $\sigma$ lines and the single cluster of $\pi$ lines can be treated as a single Doppler-broadened line shifted from the laboratory-frame wavelength by the statistically weighted average Zeeman shift of the individual lines in the cluster; this shift is zero for the case of the $\pi$ lines. By injecting the laser light parallel or perpendicular to the background magnetic field, parallel and perpendicular VDFs can be obtained. When injection is parallel, the laser light is circularly polarized so that only one cluster of $\sigma$ lines is excited. When the injection is perpendicular, the laser light is polarized parallel to the magnetic field so that only the $\pi$ lines are excited.

The LIF data presented in this work was obtained with a Sirah MatisseDR tunable ring dye laser, which has a line width of less than $20 \mathrm{MHz}$ RMS and which is pumped with a $10 \mathrm{~W}$ Spectra-Physics Millenium Pro 10s diode laser. The Millenium Pro 10s pumps the Matisse-DR using Rhodamine 6G dye which fluoresces from $550 \mathrm{~nm}$ to $660 \mathrm{~nm}$. For argon LIF in HELIX, the Matisse-DR laser is tuned to $611.6616 \mathrm{~nm}$ (vacuum wavelength) to excite the Ar II $3 \mathrm{~d}^{2} \mathrm{G}_{9 / 2}$ metastable state to the $4 \mathrm{p}^{2} \mathrm{~F}_{7 / 2}$ state, which decays to the $4 \mathrm{~s}^{2} \mathrm{D}_{5 / 2}$ state through emission of $460.96 \mathrm{mn}$ photons (shown in Fig. 7.1). Approximately $5 \%$ of the Matisse-DR output is split off using a beamsplitter for diagnostic purposes. This diagnostic beam passes through an iodine 
reference cell for a consistent zero-velocity measurement. A photodiode detects the fluorescent emission from the iodine cell during every scan of the dye laser. A Bristol Instruments 621-VIS wavelength meter also measures the wavelength of this diagnostic beam. The remaining Matisse-DR output passes through a mechanical chopper and into a single mode, non-polarization preserving optical fiber which transports the laser light into injection optics mounted on the HELIX-LEIA system. A $2.54 \mathrm{~cm}$ collimating lens coupled to a multimode optical fiber with a matching numerical aperture $(\mathrm{NA}=0.22)$ make up the collection optics. The output of this multimode optical fiber is filtered using a $\sim 1 \mathrm{~nm}$ bandwidth narrowband filter at $461 \mathrm{~nm}$ and coupled into a Hamamatsu high-gain photomultiplier tube (PMT). Since the PMT signal is composed of fluorescent radiation and electron noise, a Stanford Research Systems SR830 lock-in amplifier referenced to the mechanical chopper in the incident beam path filters the LIF emission. While the lockin amplifier output is simply recorded as a function of laser frequency in steady-state plasmas, for the pulsed plasmas in this work the lock-in output is recorded with a digitizer and the PMT output is averaged over multiple plasma pulses. The time resolution of these LIF measurements is limited by the 1 ms integration time of the lock-in amplifier. 


\subsection{Time-Resolved LIF Measurements}

The best time resolution achievable in time-resolved LIF is determined by the lifetime of the optically pumped excited state; which is on the order of a few nanoseconds for the scheme used in this work. However, limitations such as the sampling speed of the data acquisition system, the RC time constants of the experimental electronics, specific plasma conditions (such as the time to establish a steady-state discharge), and collecting enough LIF photons for a good signal-to-noise ratio prevent experimental work from achieving such resolution. For a detailed discussion of time-resolved LIF techniques developed by the WVU HELIX group, see Biloiu et al. ${ }^{[61]}$ The lock-in amplifier used to improve the signal-to-noise ratio in these experiments has a $1 \mathrm{~ms}$ integration time; which was the most limiting factor in the time resolution of this work. In addition to using a lock-in amplifier, the signal-to-noise ratio can be improved by averaging several individual measurements together. To do this, the laser frequency is held fixed at a single step in the IVDF measurement while a large number of LIF measurements are obtained. Those measurements are averaged together and then the laser is moved to the next frequency step in the IVDF and the process is repeated. In this work, each IVDF is comprised of an average of 100 separate measurements at each wave- 
length point in the IVDF. A typical time-resolved LIF measurement of the plasma studied in this work is shown in Figure 7.4. All LIF measurements presented here have a $1 \mathrm{~ms}$ time resolution, as discussed at the end of Section 7.1.

Once the time-resolved IVDF has been measured and recorded (a complete scan could take anywhere from 4 to 13 hours, depending on the laser stability) at a specific radial location for both cases when the wave perturbation is present and when it is not, the time-resolved ion temperature can be extracted. Figures 7.5- 7.16 show the ion temperature measurements and the normalized difference between those measurements at five spatial locations over the entire plasma pulse. It is important to note that while there is a suggestion of ion heating during the perturbation period at $r=0 \mathrm{~cm}$, shown in Figures 7.5 and 7.6, there is no indication of ion heating due to the perturbation at any other location. At $r=0.25 \mathrm{~cm}$, the ion temperature appears to be slightly hotter in the unperturbed case than the perturbed case (see Figures 7.7 and 7.8$)$. At $r=0.5 \mathrm{~cm}$, there is no significant difference between the two cases at any time in the plasma pulse, as shown in Figures 7.9- 7.12. At $r=0.75 \mathrm{~cm}$, as shown in Figures 7.13 and 7.14 the ion temperature does not change during the perturbation pulse, although it does significantly in- 


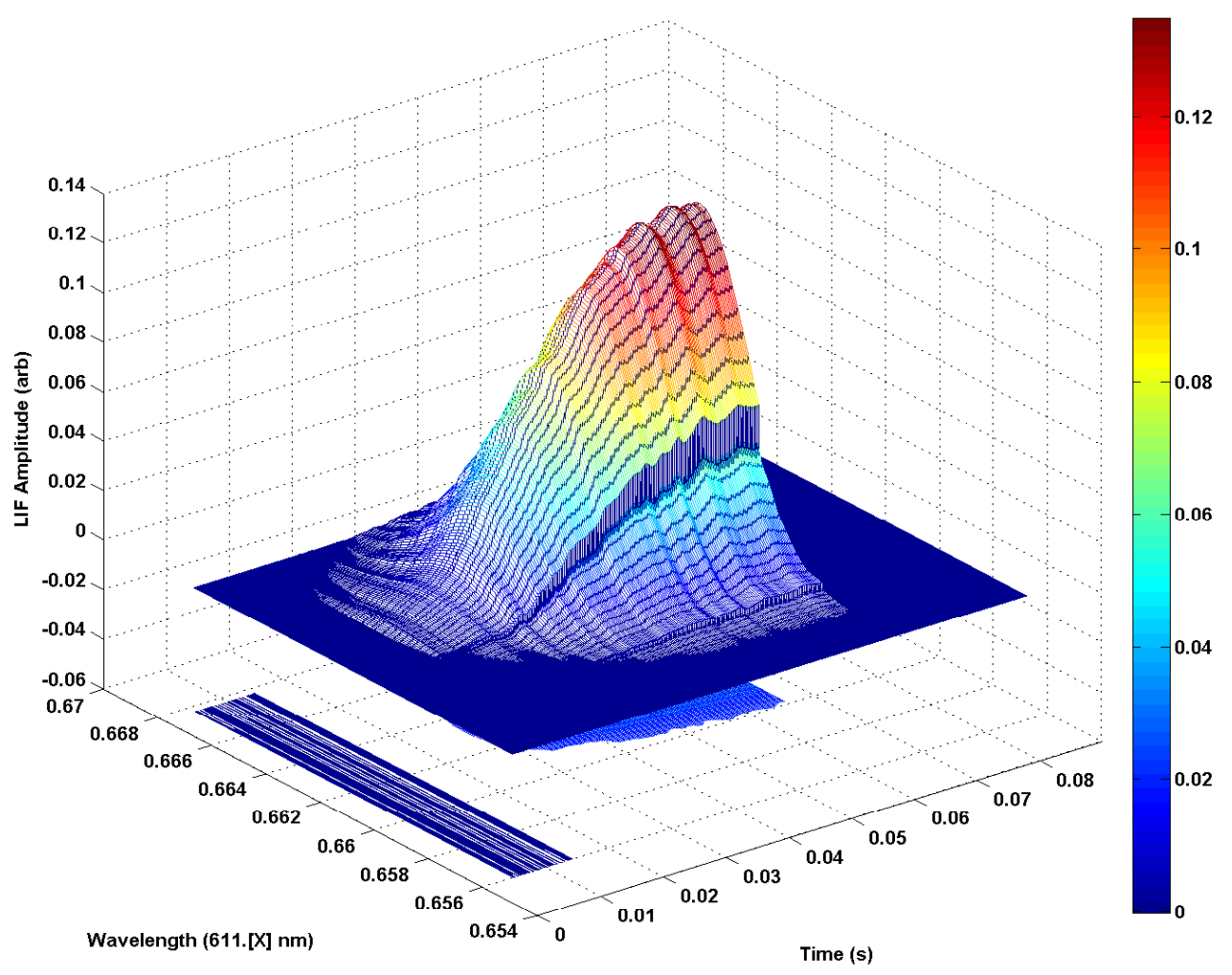

Figure 7.4: Example of an LIF-determined argon IVDF during a $60 \mathrm{~ms}$ plasma pulse with $1 \mathrm{~ms}$ time resolution. This measurement was taken at the center of the plasma column $(r=0 \mathrm{~cm})$ while the wave-launching antenna was on (the wave pulse lasts from $32.5-40.5 \mathrm{~ms}$ in the pulse). 
crease at the end of the plasma pulse ( $t=50 \mathrm{~ms}-t=60 \mathrm{~ms})$. Why this happens is not understood. At $r=1 \mathrm{~cm}$, the LIF signal was very weak and difficult to fit well, resulting in larger error. Within the experimental error at the location, there is no difference between the perturbed and unperturbed ion temperatures. It is possible that due to the weakness of the perturbation signal the ion response is difficult to distinguish from random fluctuations in the plasma. The best way to confirm this is to repeat the experiment with a larger amplitude perturbation to see if ion heating can be measured at more locations or if there is no ion heating at all in response to this perturbation at larger radii. A helpful summary of how the ion temperature response changes across the plasma column is shown in Figure 7.17. At $r=0 \mathrm{~cm}$ the normalized difference in the ion temperature is statistically significant (greater than $10 \%$ ) while at larger radii it is not. Due to the low signal-to-noise ratio at $r=1 \mathrm{~cm}$ and the poor temperature fit, the normalized temperature difference is not shown for this location. 


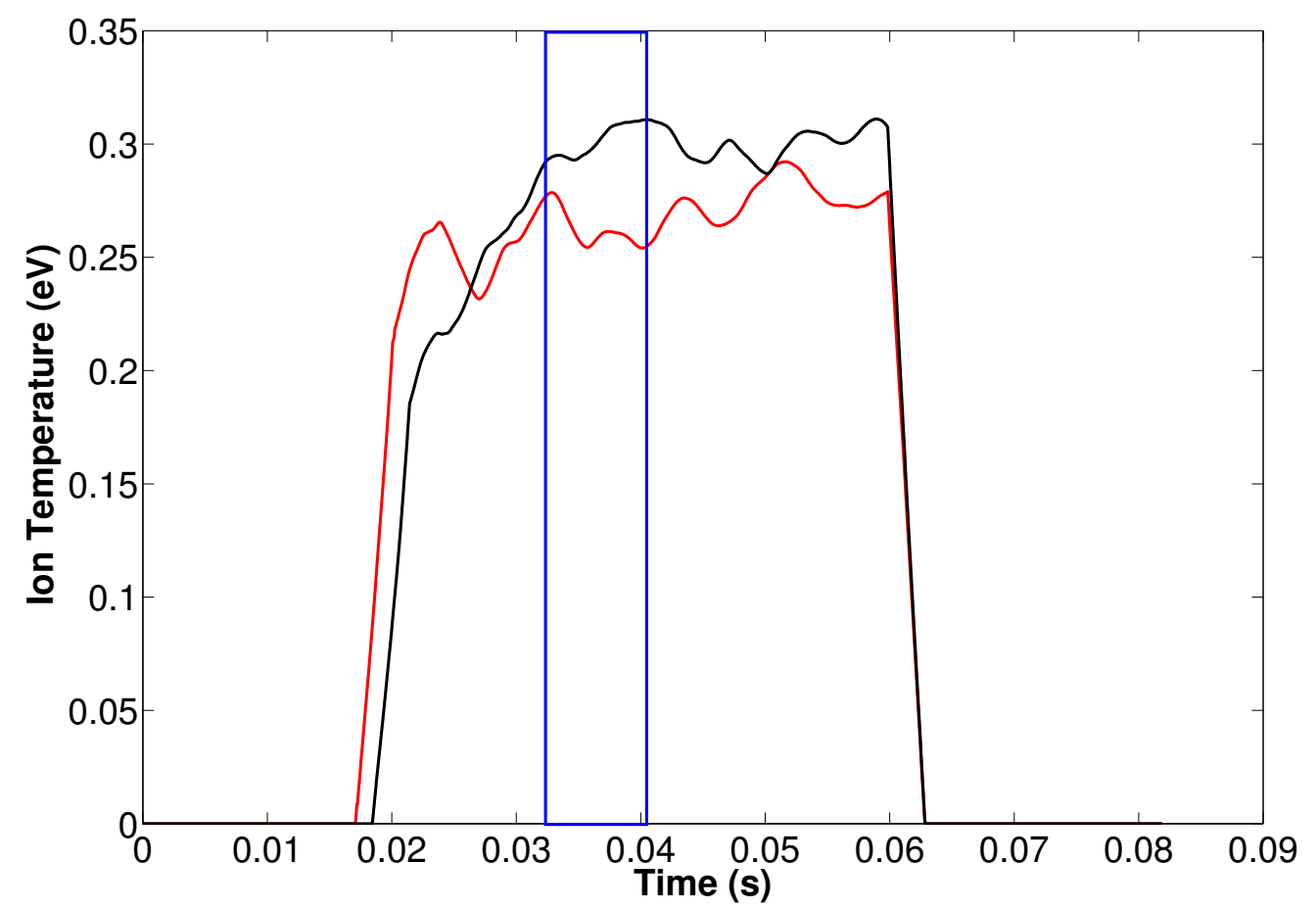

Figure 7.5: Ion temperature during the plasma pulse at $r=0 \mathrm{~cm}$. The red line is with no perturbation, the black line is with perturbation, and the blue box is the $8 \mathrm{~ms}$ pulse (32.5-40.5 ms) when the perturbation is present. Notice that at this location, there is a suggestion of ion heating during the perturbation pulse. 


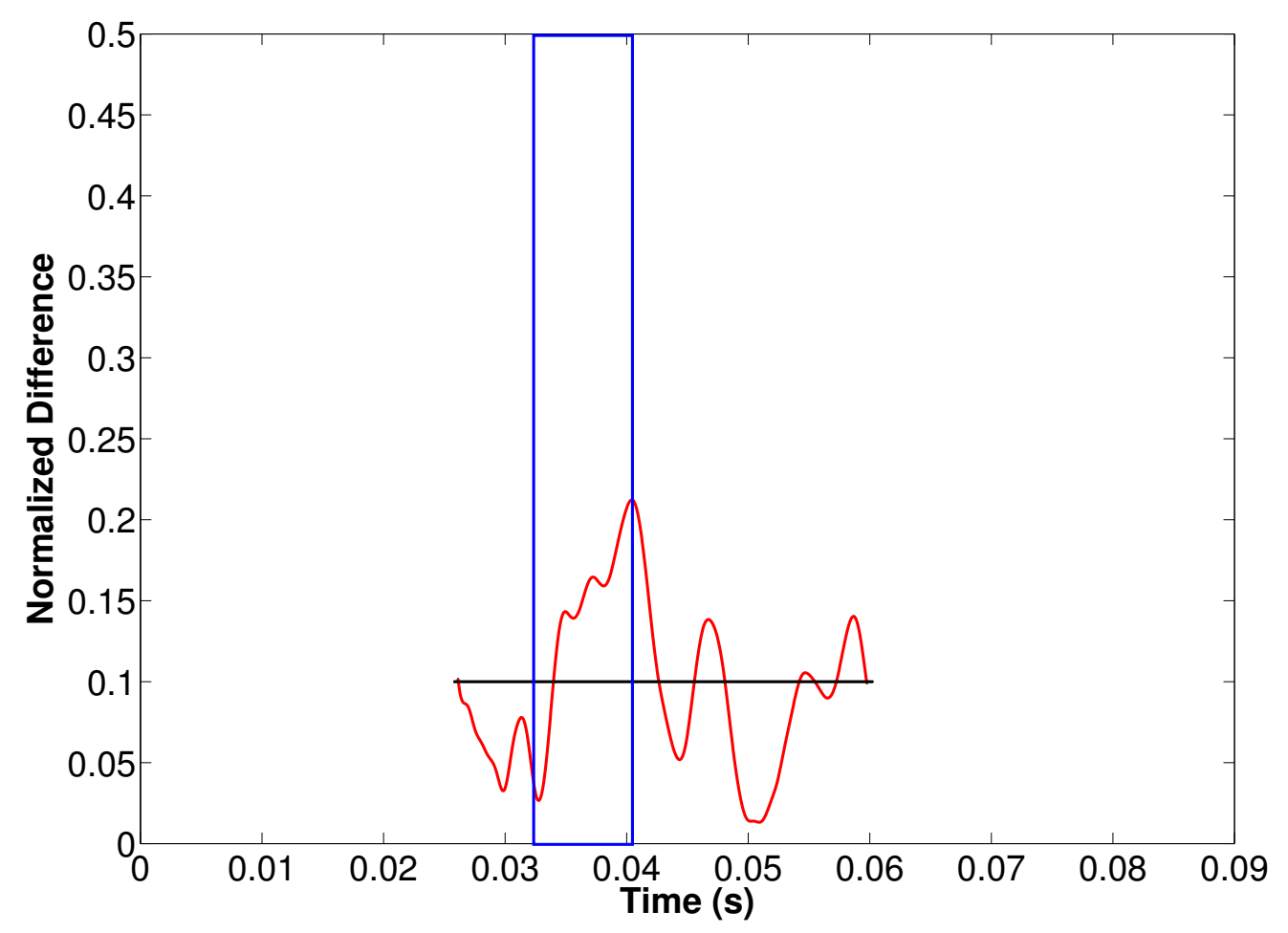

Figure 7.6: Normalized difference plot for the ion temperature measurement shown in Figure 7.5. The red line is the normalized difference between the wave-on and wave-off measurements. The black line shows where the normalized difference is equal to $10 \%$ of the average value of the two curves. A normalized difference less than $10 \%$ is smaller than the experimental error in the LIF measurements presented here and indicates that the two cases are similar enough to be considered the same. At this radial location, the normalized difference is above $10 \%$ during the end of the perturbation pulse, indicating that the observed ion heating in Figure 7.5 is real. 


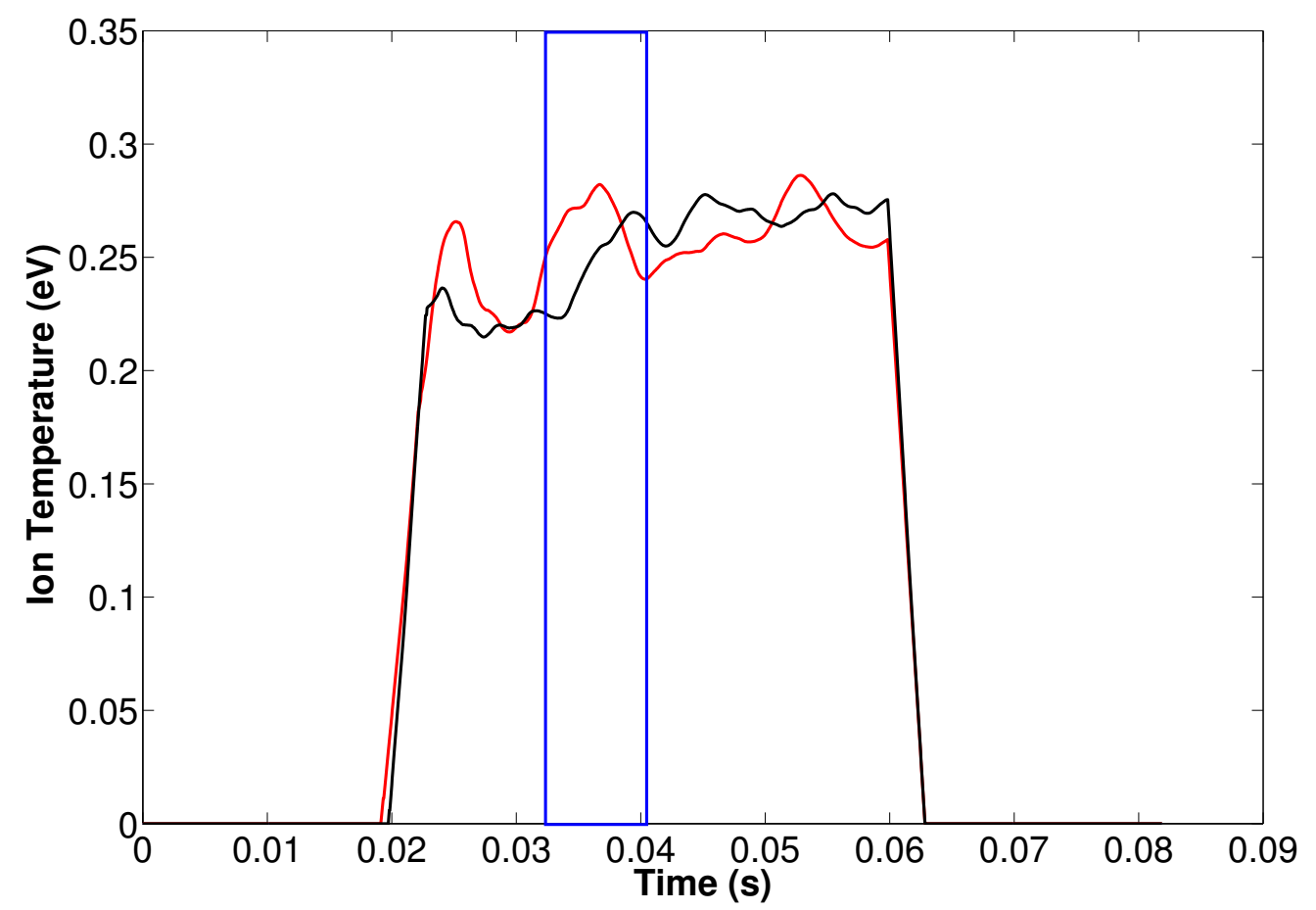

Figure 7.7: Ion temperature during the plasma pulse at $r=0.25 \mathrm{~cm}$. The red line is with no perturbation, the black line is with perturbation, and the blue box is the $8 \mathrm{~ms}$ pulse (32.5-40.5 ms) when the perturbation is present. At this radial location it is difficult to see any ion heating caused by the perturbation pulse. 


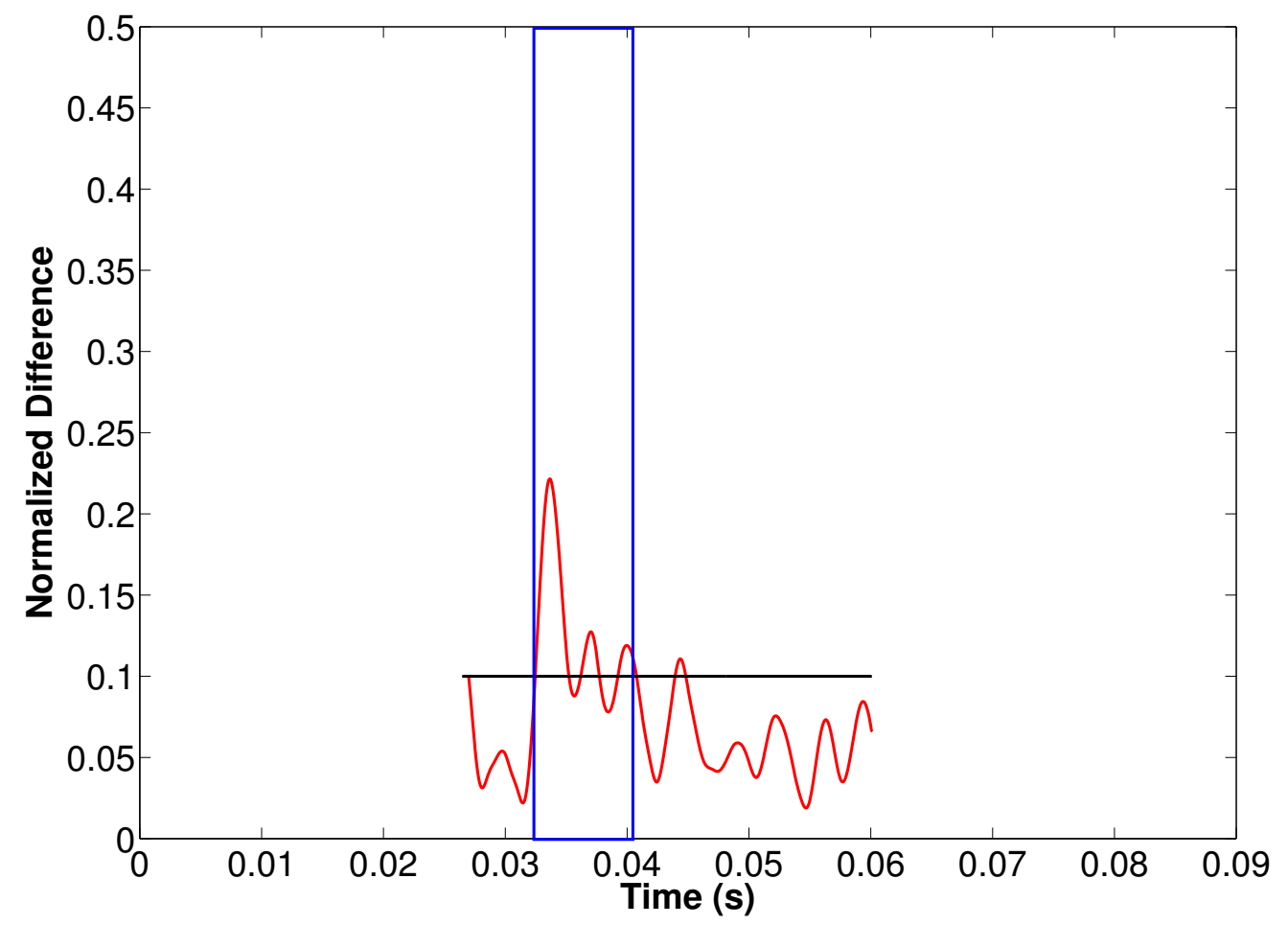

Figure 7.8: Normalized difference plot for the ion temperature measurement shown in Figure 7.7. At the very beginning of the perturbation pulse, the normalized percent difference hints at the ion temperature being hotter without the wave perturbation than with it. 


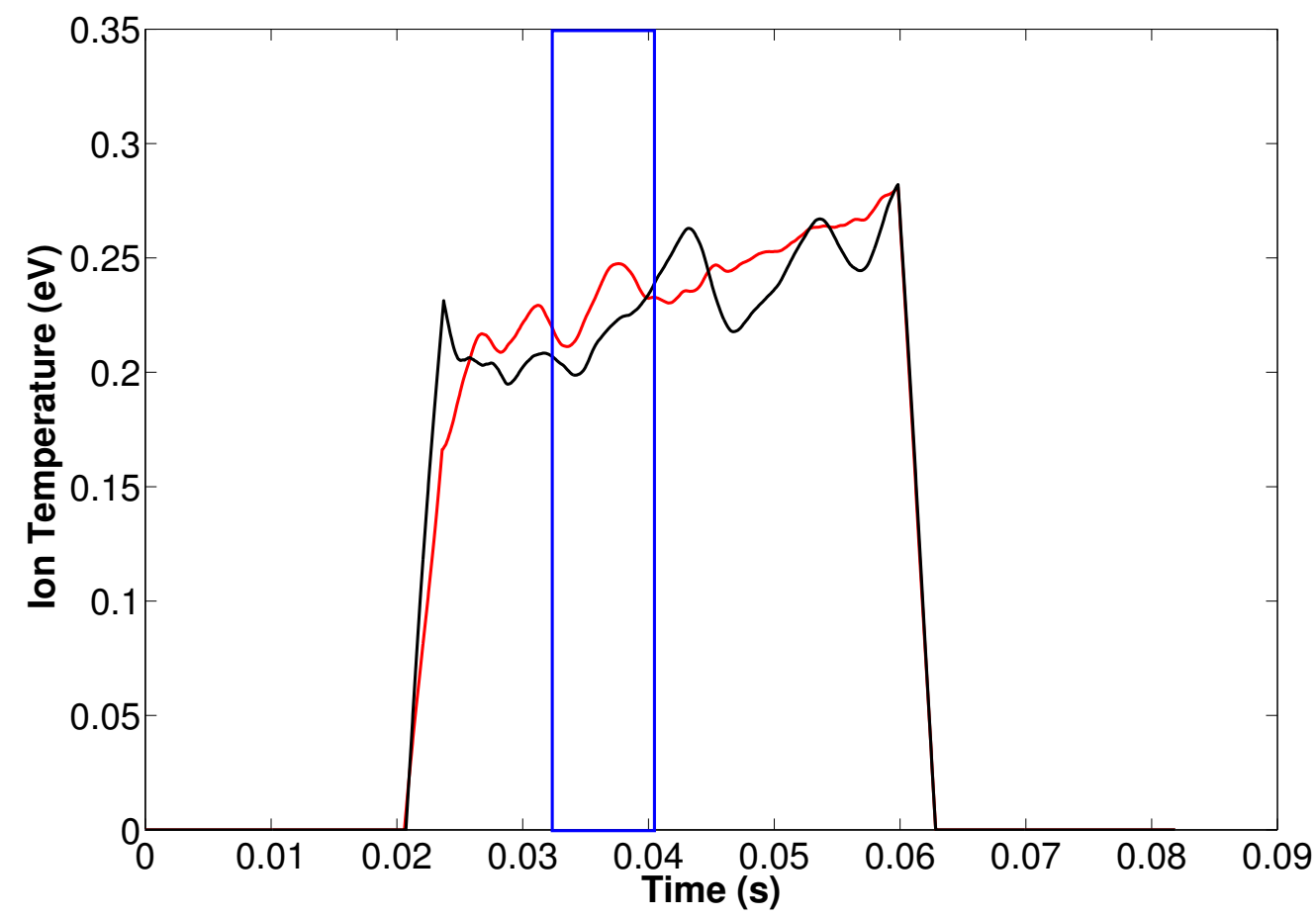

Figure 7.9: Ion temperature during the plasma pulse at $r=0.50 \mathrm{~cm}$. The red line is with no perturbation, the black line is with perturbation, and the blue box is the $8 \mathrm{~ms}$ pulse $(32.5-40.5 \mathrm{~ms})$ when the perturbation is present. At this radial location there is no appreciable difference between the temperature behavior in either case. 


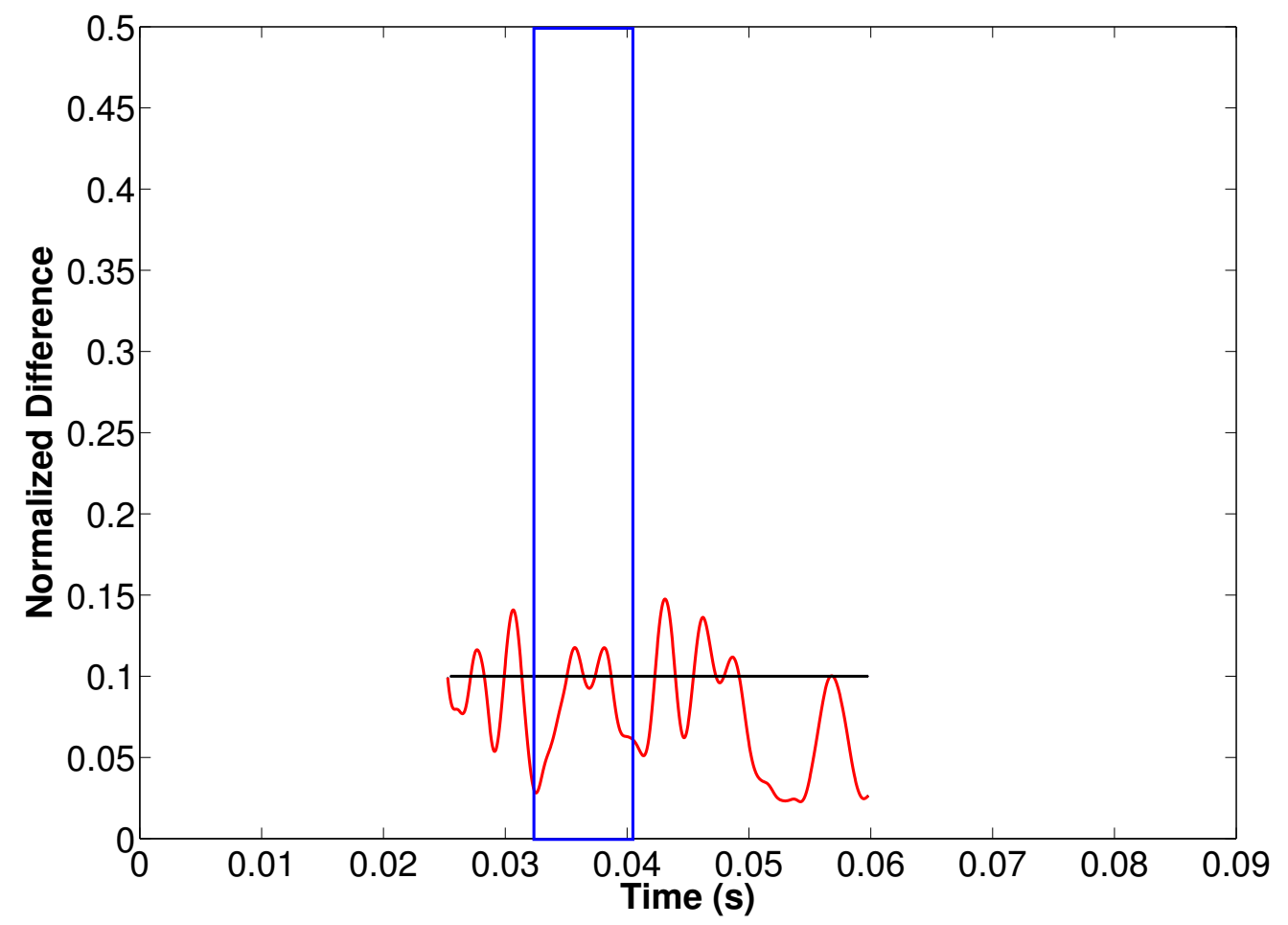

Figure 7.10: Normalized difference plot for the ion temperature measurement shown in Figure 7.9. At this radial location, the normalized difference between the two cases oscillates around $10 \%$, indicating that there is no significant change in the ion temperature due to the wave perturbation. 


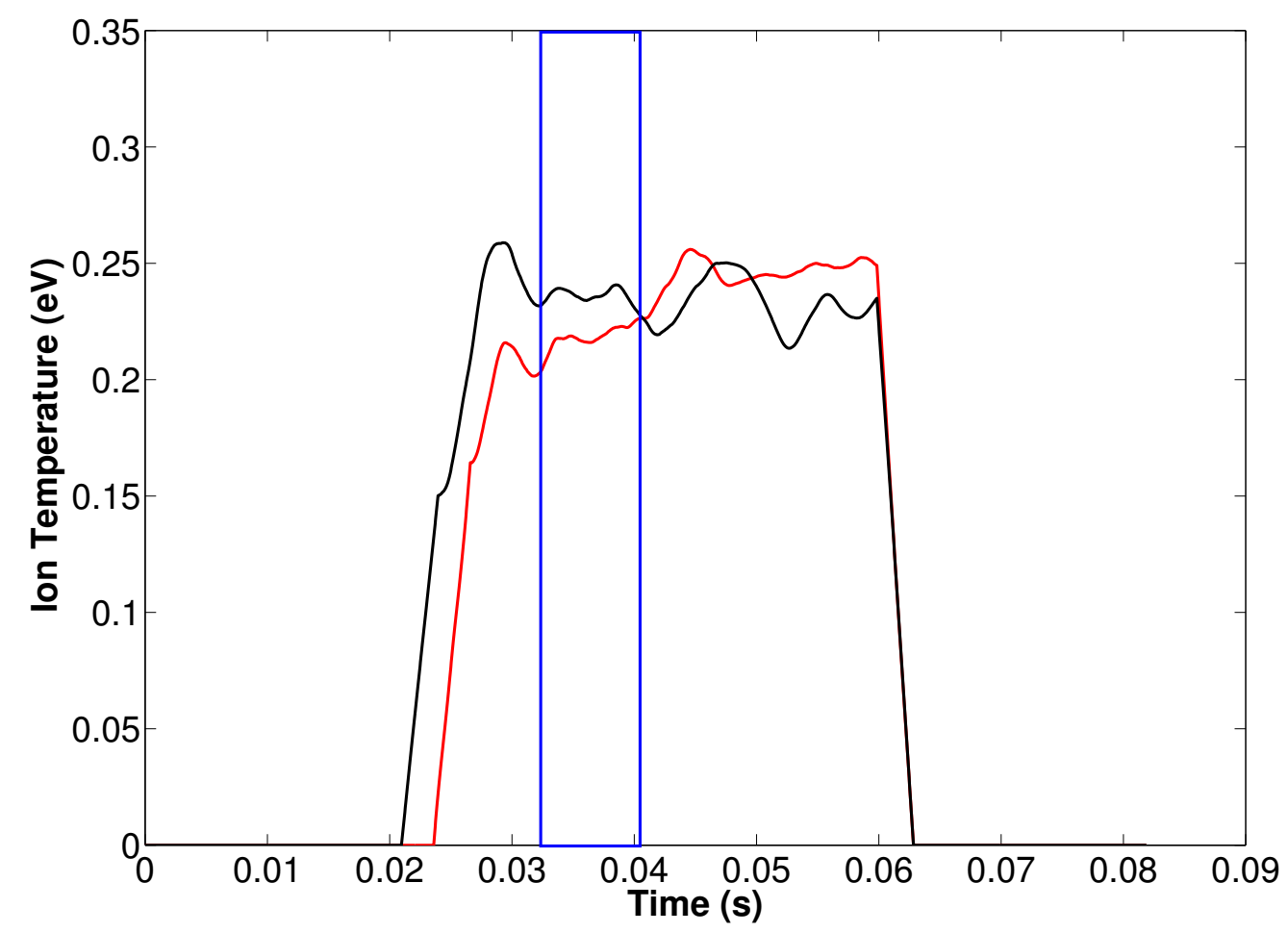

Figure 7.11: Ion temperature during the plasma pulse at $r=0.50 \mathrm{~cm}$, taken on a different day than the data shown in Figure 7.9 to make sure the data were reproducible. The red line is with no perturbation, the black line is with perturbation, and the blue box is the $8 \mathrm{~ms}$ pulse (32.5-40.5 ms) when the perturbation is present. As in the previous figure, there is no appreciable difference between the ion temperature behavior in the two cases. 


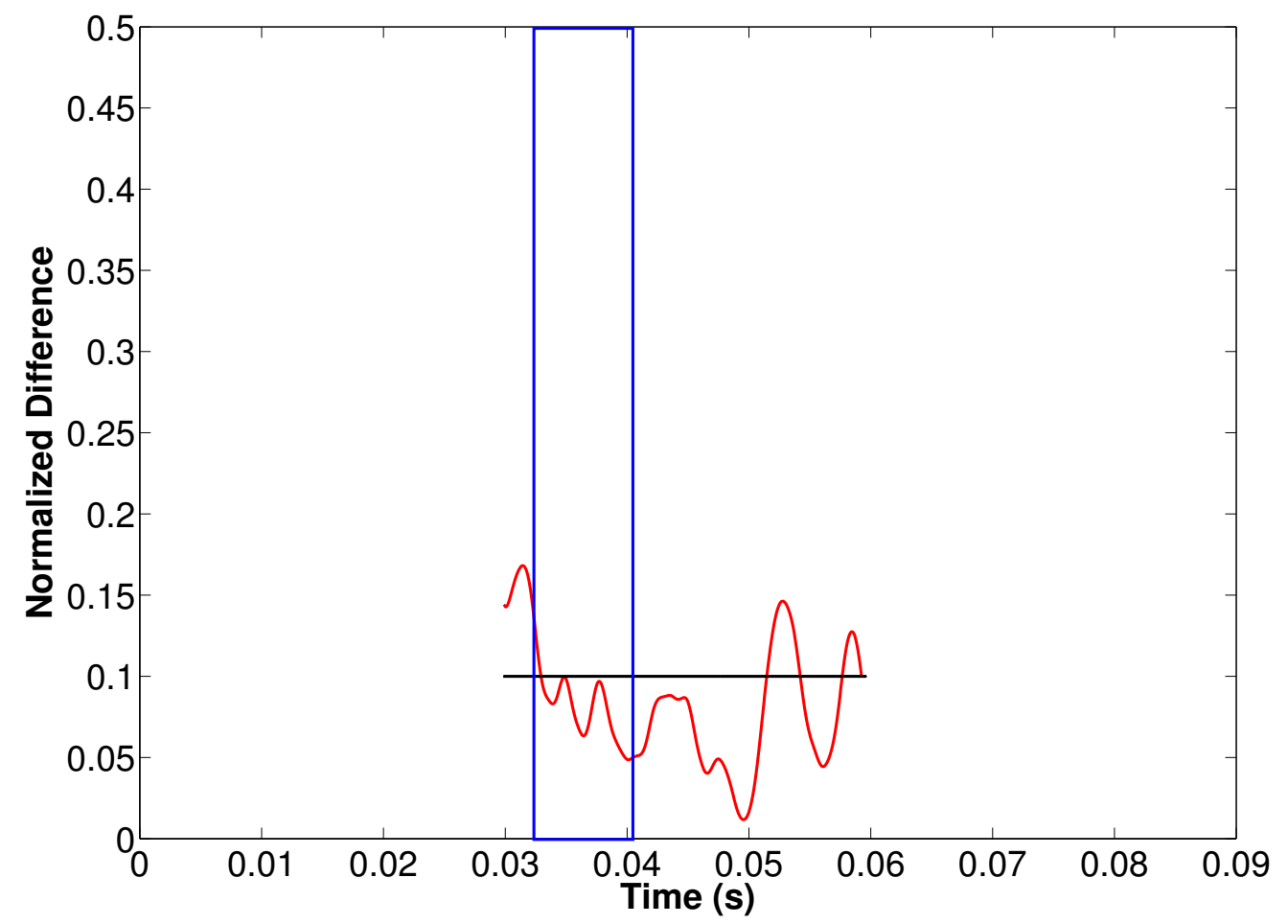

Figure 7.12: Normalized difference plot for the ion temperature measurement shown in Figure 7.11. As in the first measurement at $r=0.50 \mathrm{~cm}$, there is no significant change in the ion temperature due to the wave perturbation. 


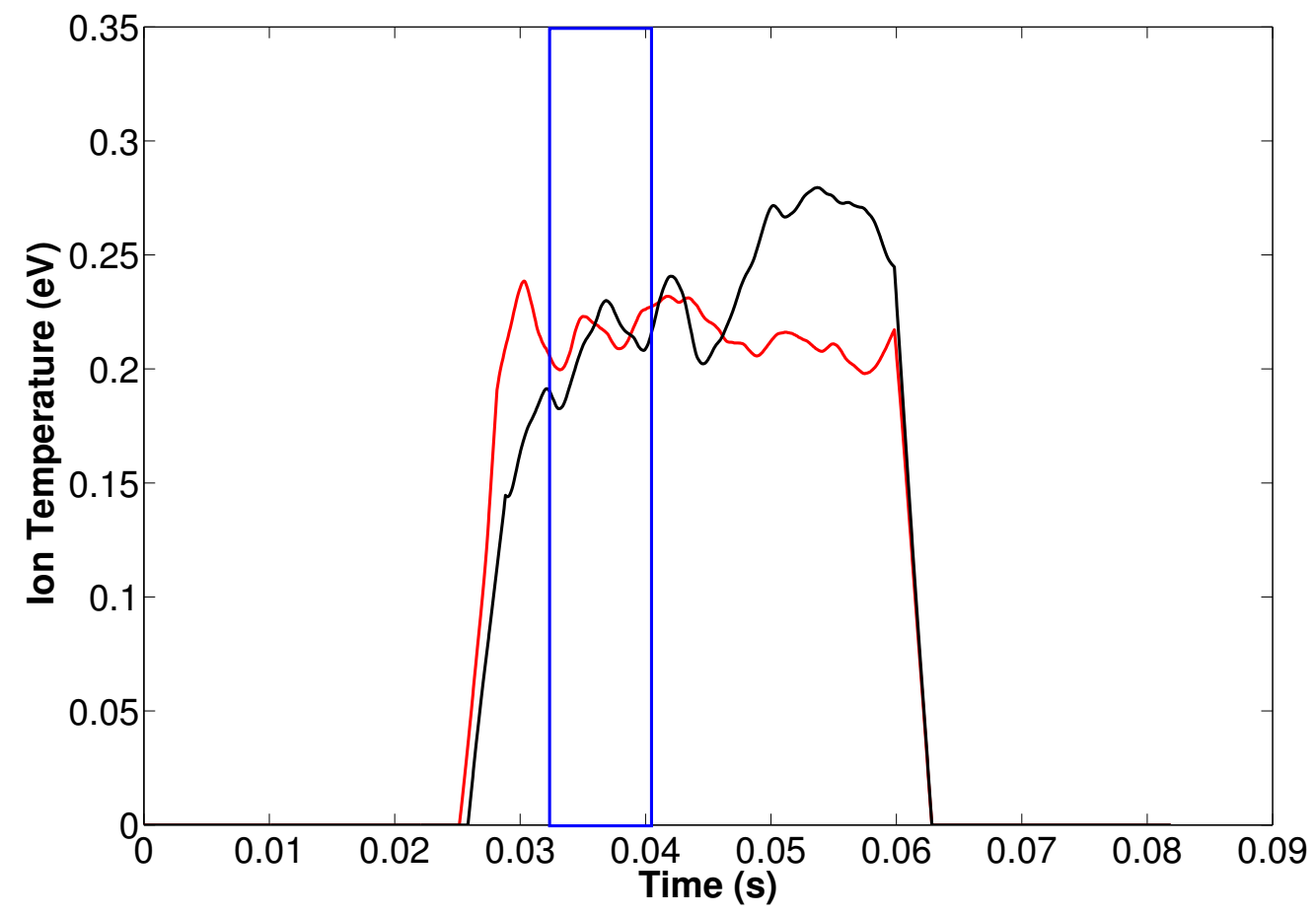

Figure 7.13: Ion temperature during the plasma pulse at $r=0.75 \mathrm{~cm}$. The red line is with no perturbation, the black line is with perturbation, and the blue box is the $8 \mathrm{~ms}$ pulse (32.5-40.5 ms) when the perturbation is present. At this radial location the ion temperature during the perturbation pulse is indistinguishable from the temperature during the steady-state observation; although the ion temperature is noticeably warmer during the last $10 \mathrm{~ms}$ of the discharge. 


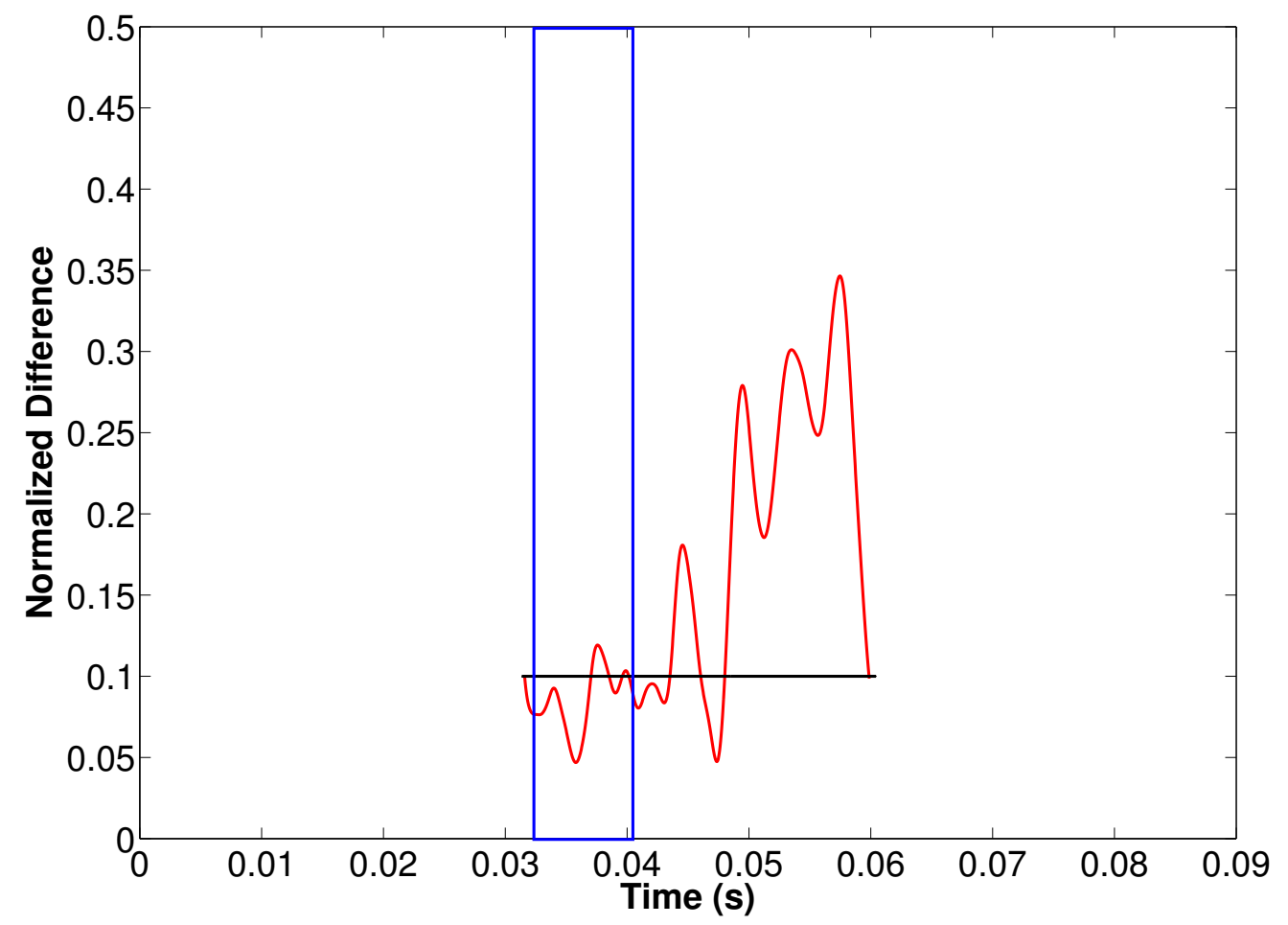

Figure 7.14: Normalized difference plot for the ion temperature measurement shown in Figure 7.13. At this radial location there is no experimentally significant difference between the two ion temperature measurements during the perturbation. 


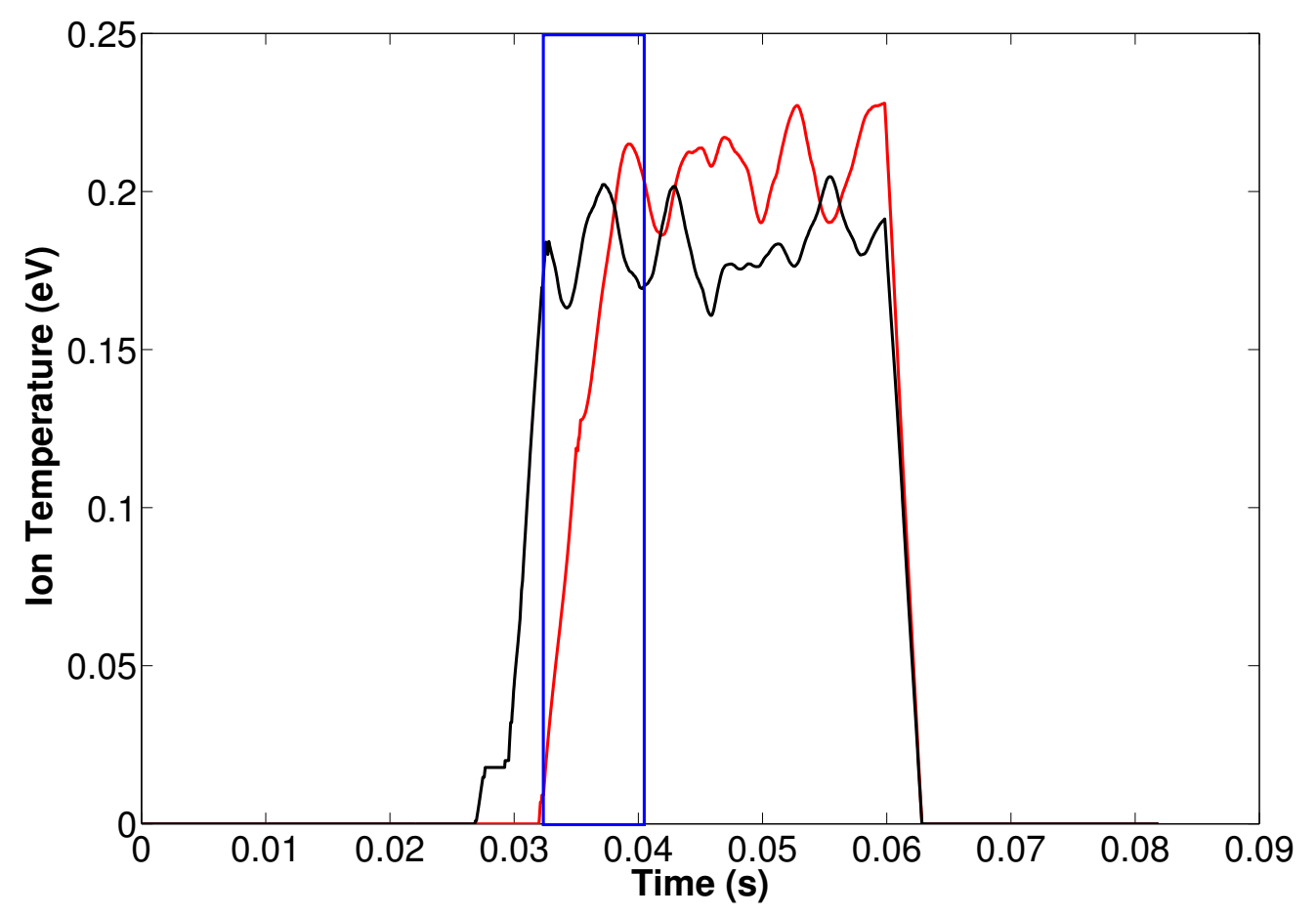

Figure 7.15: Ion temperature during the plasma pulse at $r=1.0 \mathrm{~cm}$. The red line is with no perturbation, the black line is with perturbation, and the blue box is the $8 \mathrm{~ms}$ pulse $(32.5-40.5 \mathrm{~ms})$ when the perturbation is present. This radial location was at the edge of the LIF detection threshold for these plasma conditions. Reliably fitting a Gaussian profile for temperature determination was difficult. At this radial location the perturbation pulse appears to raise the ion temperature faster than the unperturbed case, but for the final 20 ms of the discharge, the unperturbed ion temperature appears to be larger. 


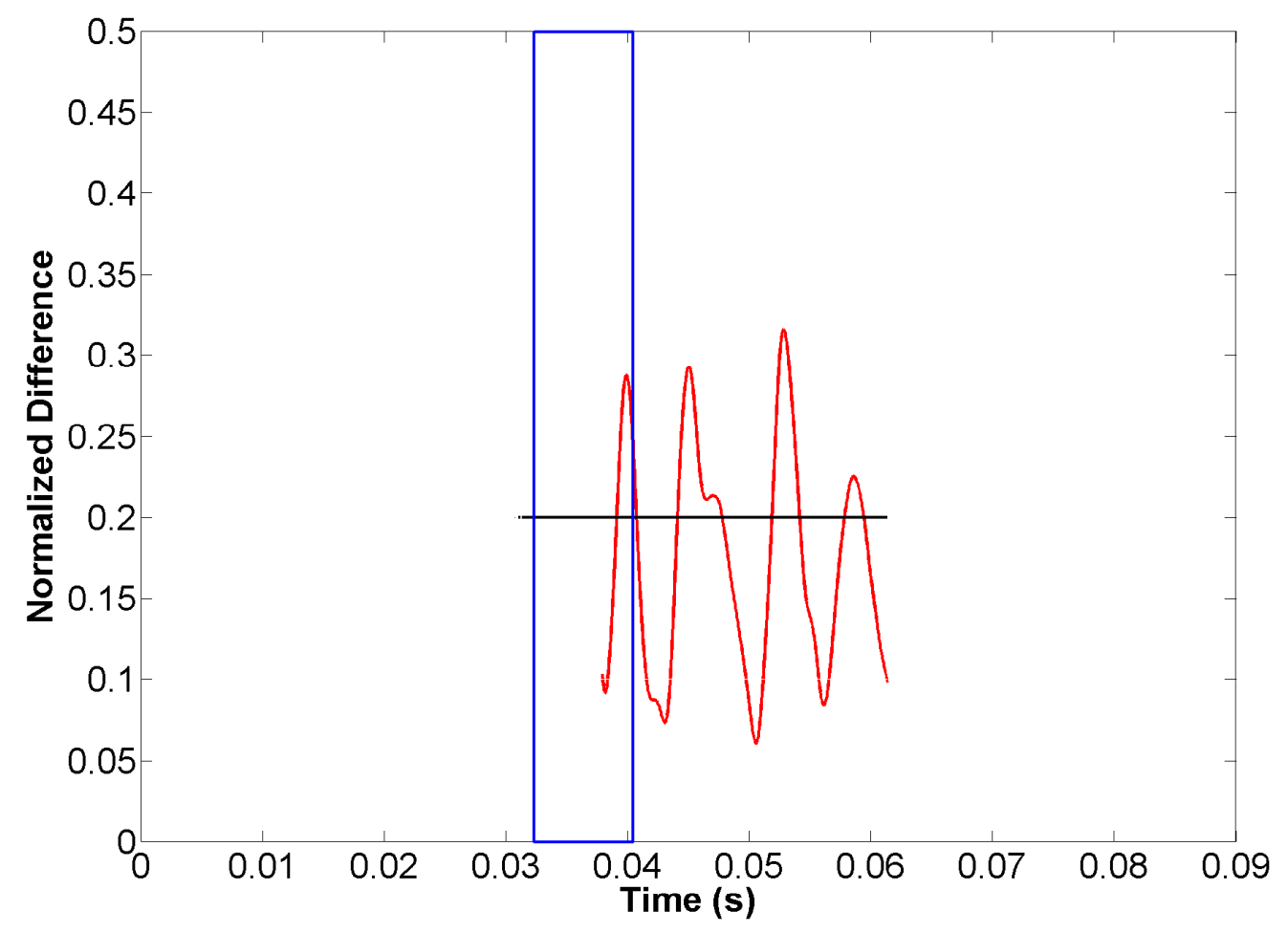

Figure 7.16: Normalized difference plot for the ion temperature measurement shown in Figure 7.15. At this radial location, proper fitting and normalization were not possible for the entire pulse due to the low LIF signal. The normalized difference fluctuates wildly (between $10 \%$ and $30 \%$ ) over the final $40 \mathrm{~ms}$ of the plasma discharge. At this location, there is no difference between the perturbed and unperturbed ion temperature separate from experimental error. 


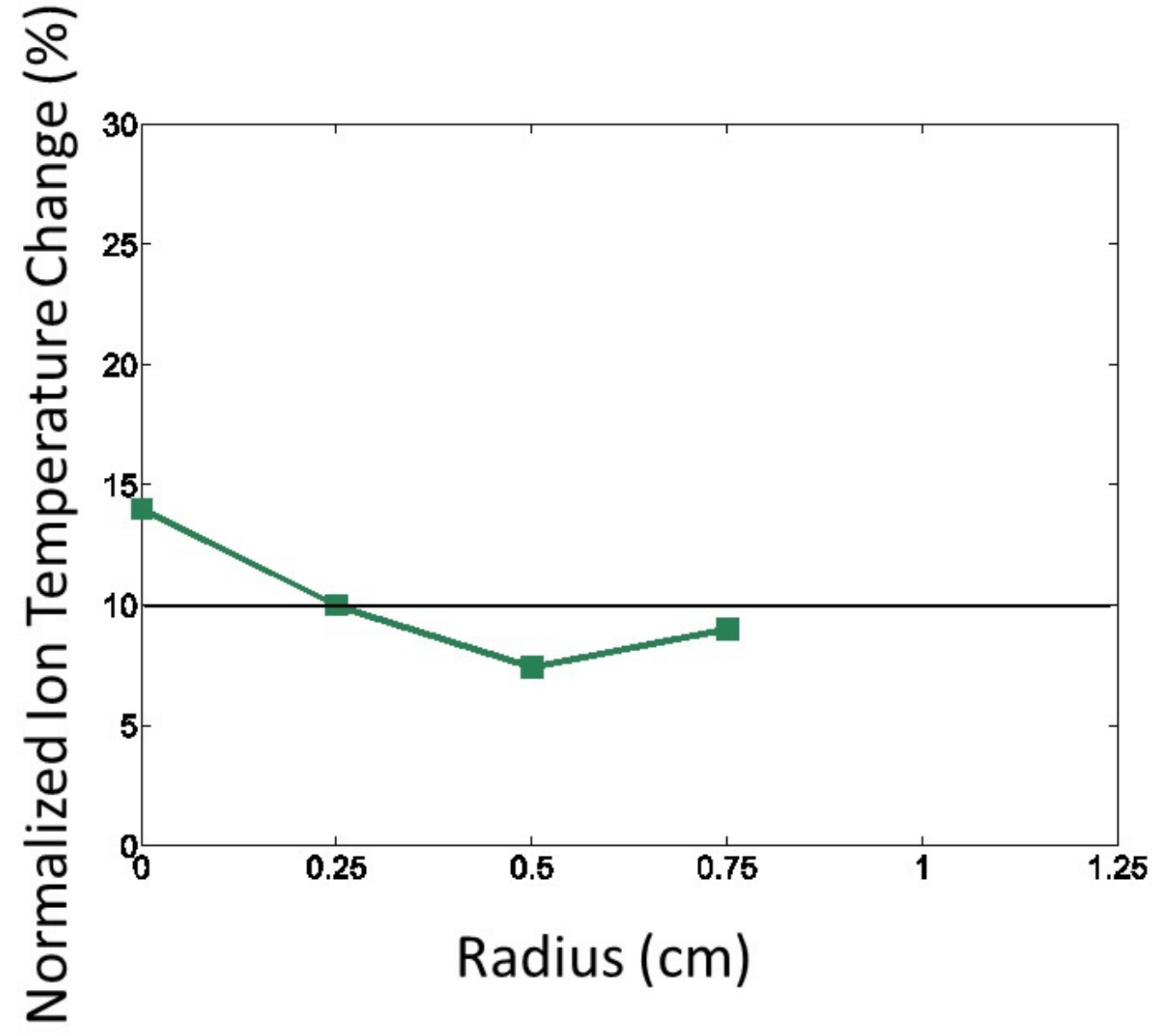

Figure 7.17: The normalized difference in the ion temperature is shown for all radial locations where reliable measurements were possible. At $r=0 \mathrm{~cm}$ the normalized difference is well above $10 \%$ and is statistically significant. For all larger radial values, the difference in ion temperature due to the wave perturbation is not statistically significant. The $10 \%$ significance threshold is indicated by the black horizontal line. Comparison of this plot with Figure 6.13 shows a correlation between the ion heating and the presence of strong turbulence. 
Since the amplitude of the LIF signal is proportional to the density of the metastable ions, any change in the LIF signal amplitude over the discharge sheds light on how the ion density behaves during that time. Figures 7.187.29 show that the metastable density is depleted during the perturbation pulse at radii larger than $0.25 \mathrm{~cm}$. At the center of the plasma column $(r=0$ $\mathrm{cm}$ ), there appears to be no change in the metastable density due to the perturbation (seen in Figures 7.18 and 7.19). As the radial location increases the metastable ion density is more strongly affected by the perturbation pulse. Similarly, Figures 7.20 and 7.21 show no appreciable difference in the LIF signal amplitude between the two cases. At $r=0.5 \mathrm{~cm}$ (shown in Figures 7.227.25), the metastable density is depleted during the perturbation pulse, but then recovers and exceeds the unperturbed case after the perturbation ends. At $r=0.75 \mathrm{~cm}$, the depletion and recovery are even more pronounced while at $r=1 \mathrm{~cm}$, the depletion due to the perturbation is the strongest and most long-lasting of all the measurements, but the recovery does not exceed the unperturbed case in the last $10 \mathrm{~ms}$ of the discharge, as it does at $r=0.50$ $\mathrm{cm}$ and $r=0.75 \mathrm{~cm}$. Notice that the vertical axes are rescaled in each figure to best display the change in signal amplitude; the maximum signal in Figure 7.18 is nearly seven times greater than that in Figure 7.28. 


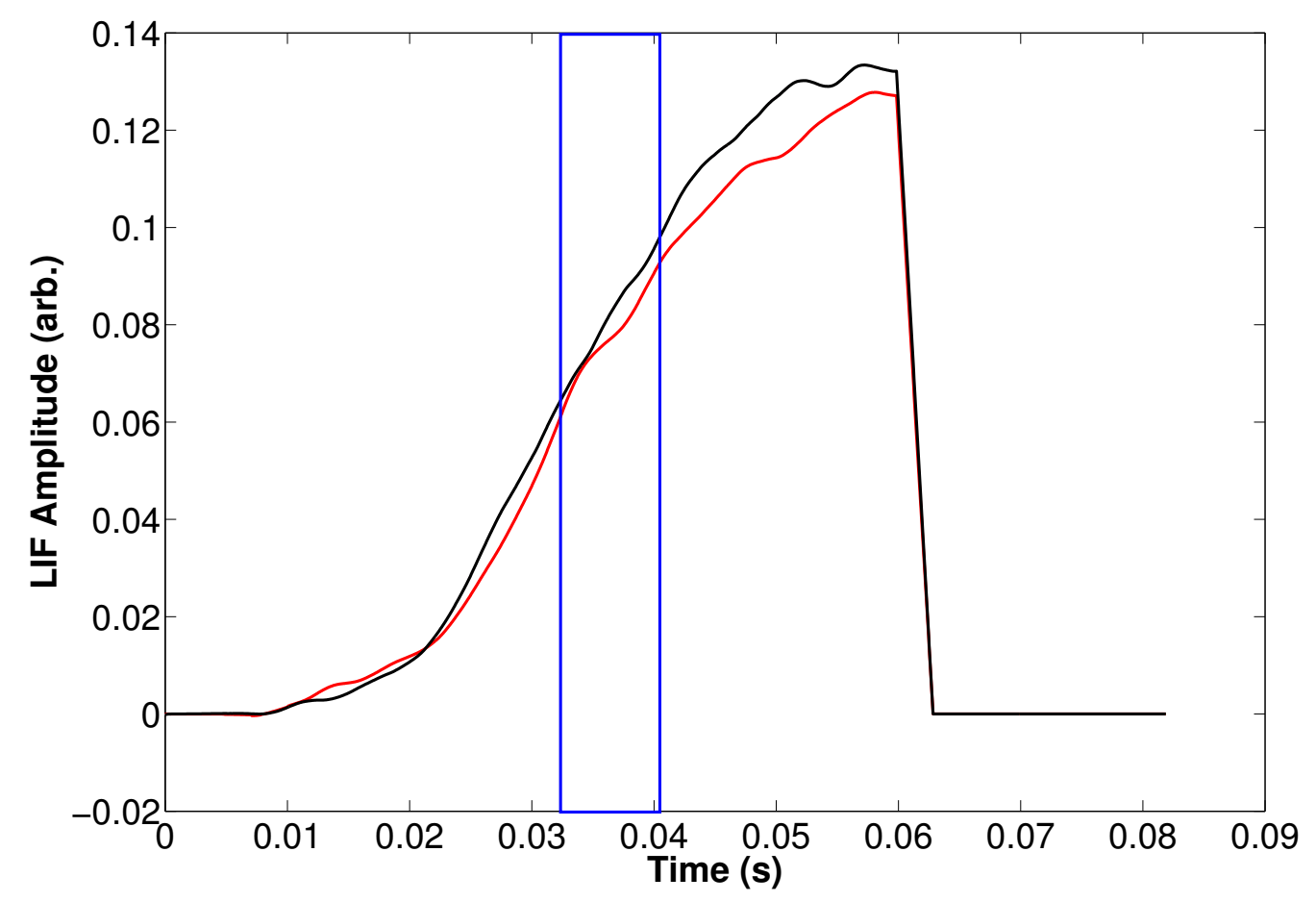

Figure 7.18: LIF signal amplitude during the plasma pulse at $r=0 \mathrm{~cm}$. The red line is with no perturbation, the black line is with perturbation, and the blue box is the $8 \mathrm{~ms}$ pulse $(32.5-40.5 \mathrm{~ms})$ when the perturbation is present. At this radial location, there is no significant difference between the perturbation and no perturbation cases. 


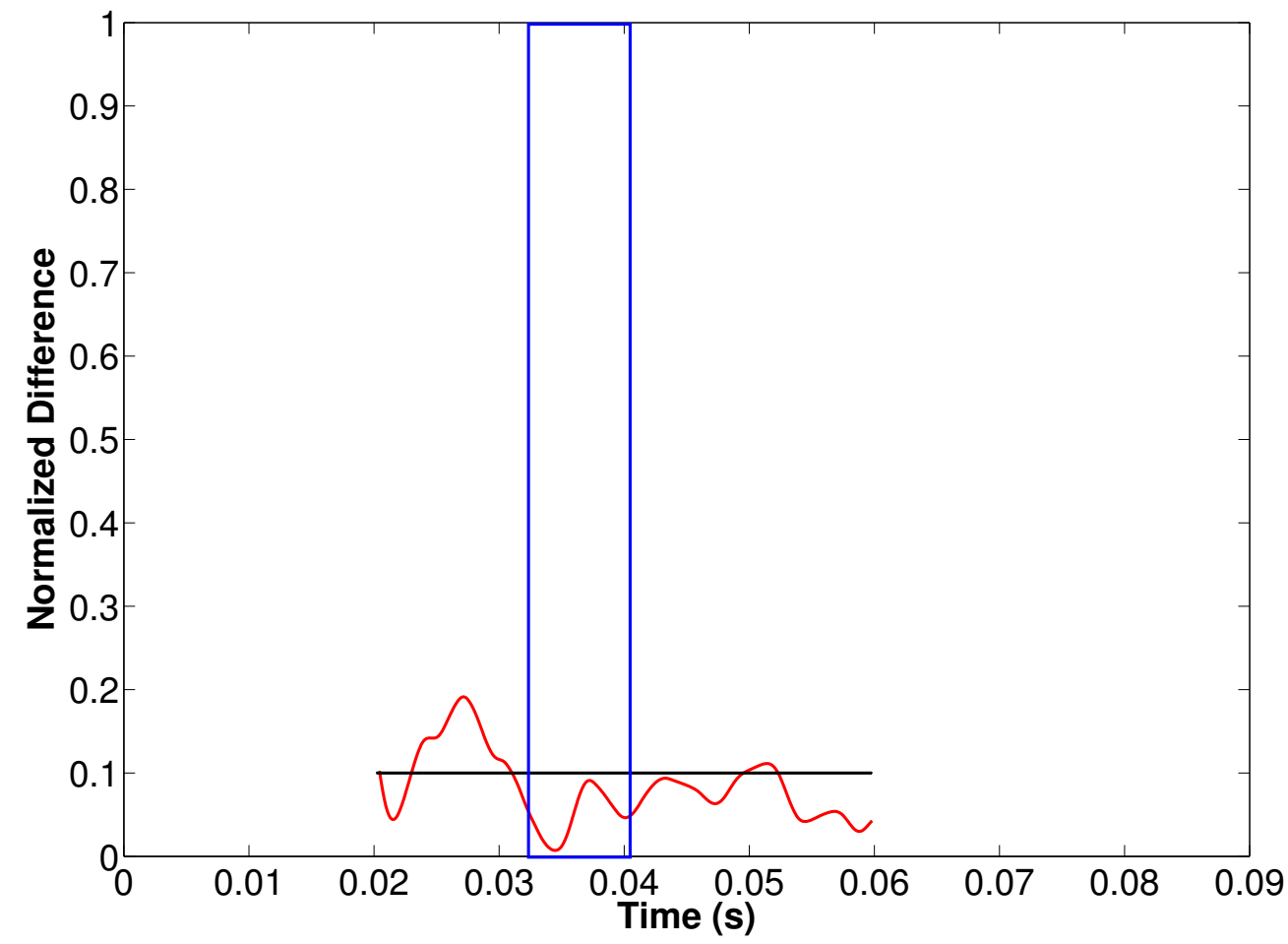

Figure 7.19: Normalized difference plot for the LIF amplitude measurement shown in Figure 7.18. At this location, there is no significant metastable density change due to the perturbation pulse. 


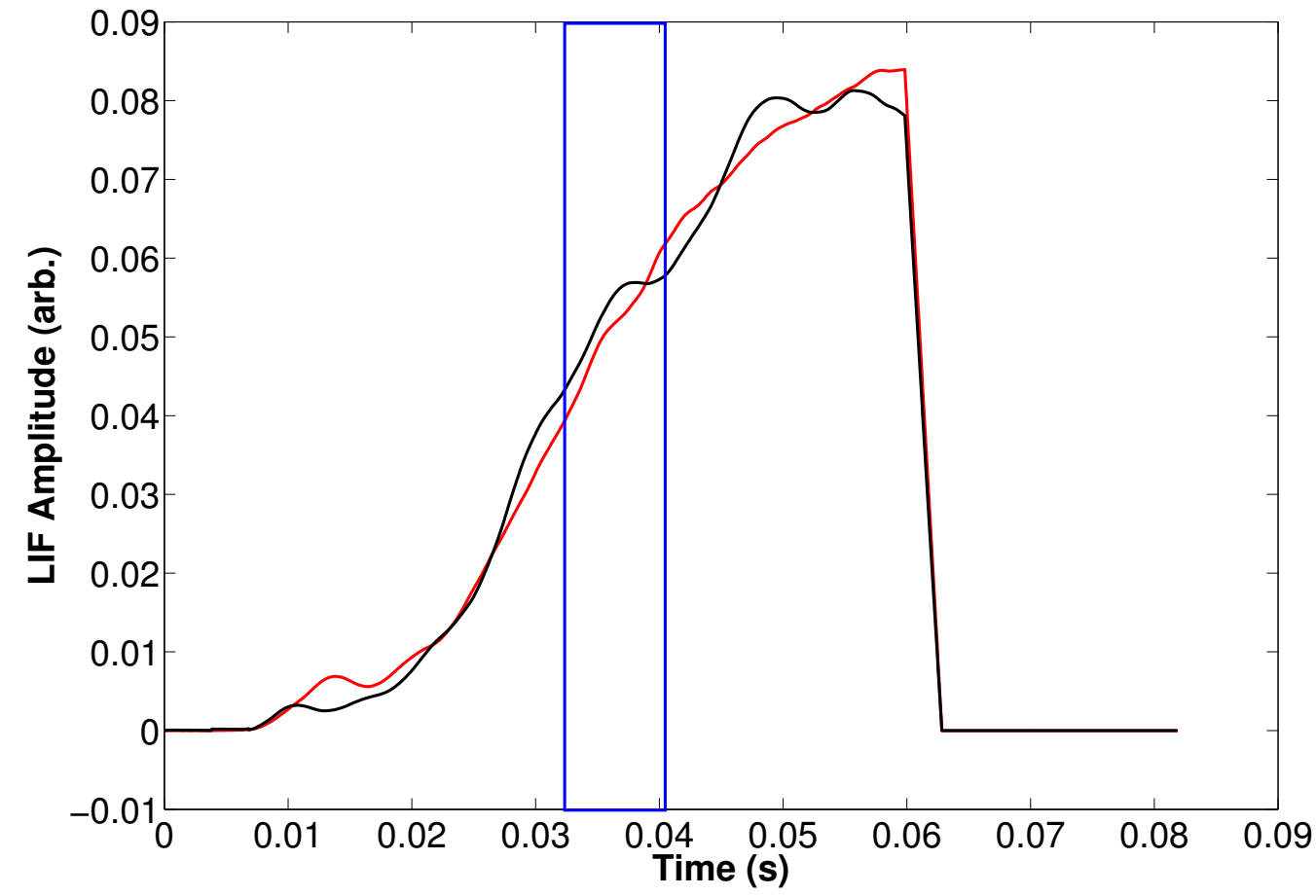

Figure 7.20: LIF signal amplitude during the plasma pulse at $r=0.25 \mathrm{~cm}$. The red line is with no perturbation, the black line is with perturbation, and the blue box is the $8 \mathrm{~ms}$ pulse $(32.5-40.5 \mathrm{~ms})$ when the perturbation is present. 


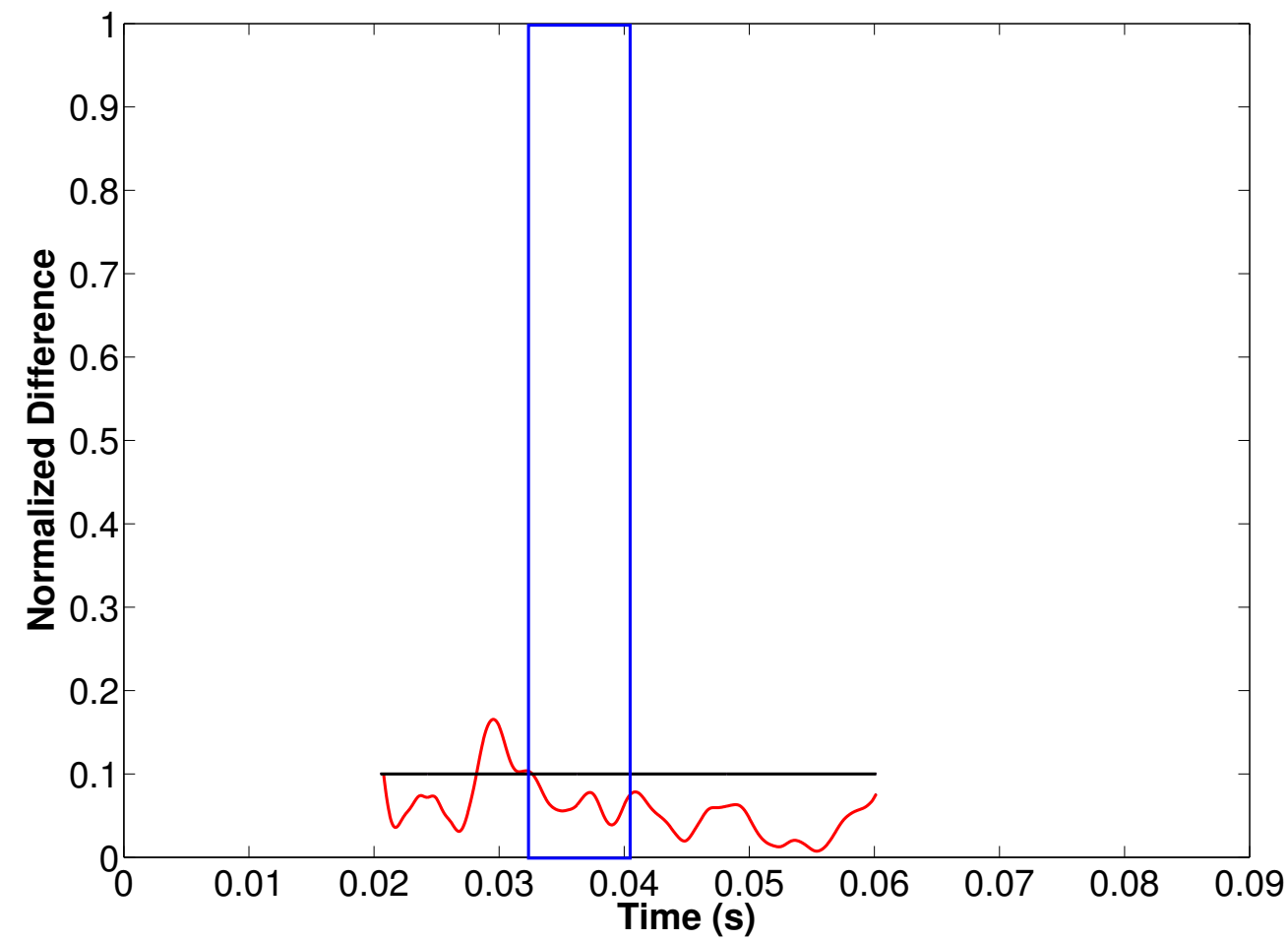

Figure 7.21: Normalized difference plot for the LIF Amplitude Measurement shown in Figure 7.20. At this radial location, there is no significant difference between the perturbed and unperturbed metastable density. 


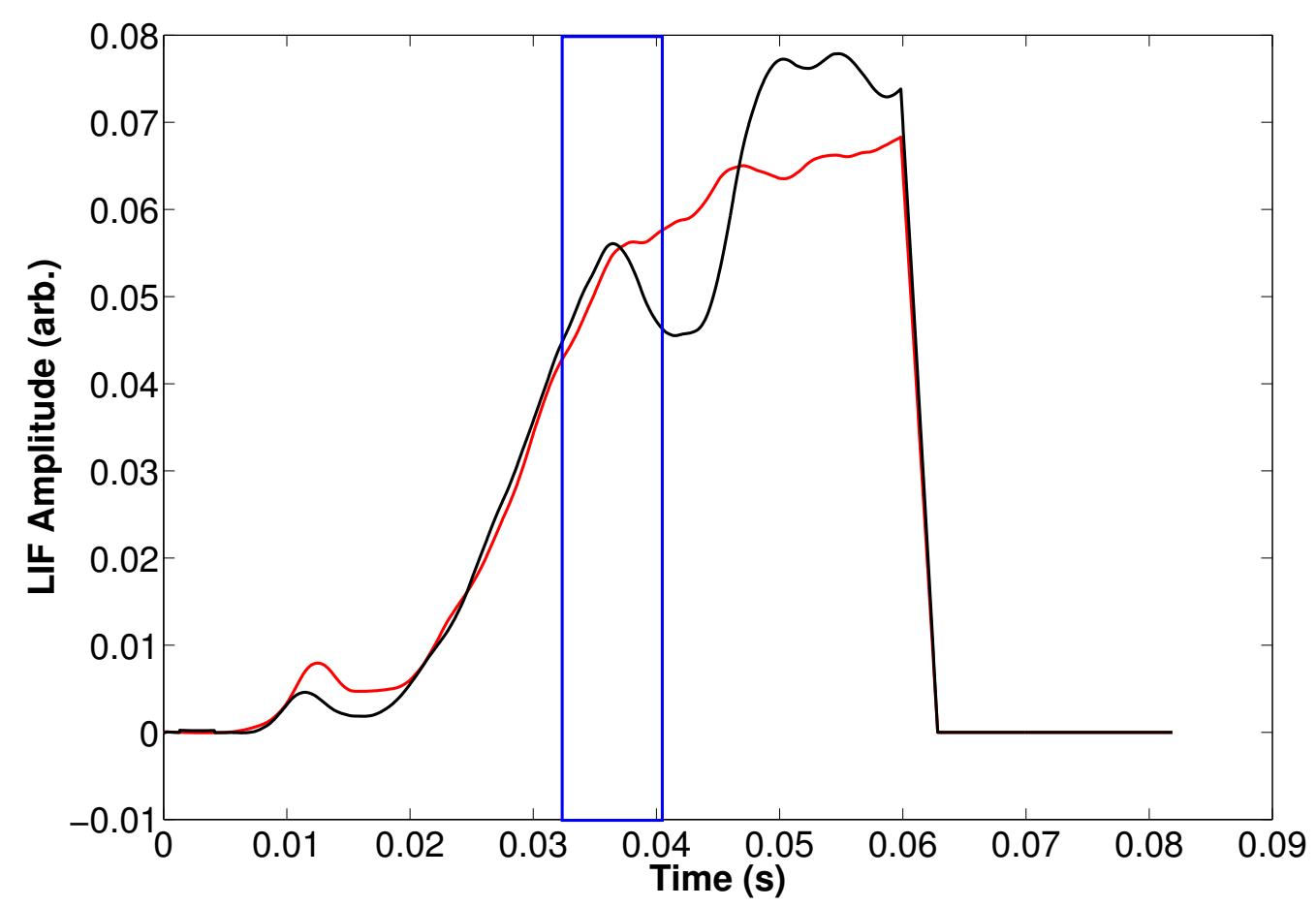

Figure 7.22: LIF signal amplitude during the plasma pulse at $r=0.50 \mathrm{~cm}$. The red line is with no perturbation, the black line is with perturbation, and the blue box is the $8 \mathrm{~ms}$ pulse $(32.5-40.5 \mathrm{~ms})$ when the perturbation is present. At this radial location the perturbation pulse significantly depletes the metastable ion population, which only begins to recover after the perturbation ends and then goes on to exceed the density in the case with no perturbation. 


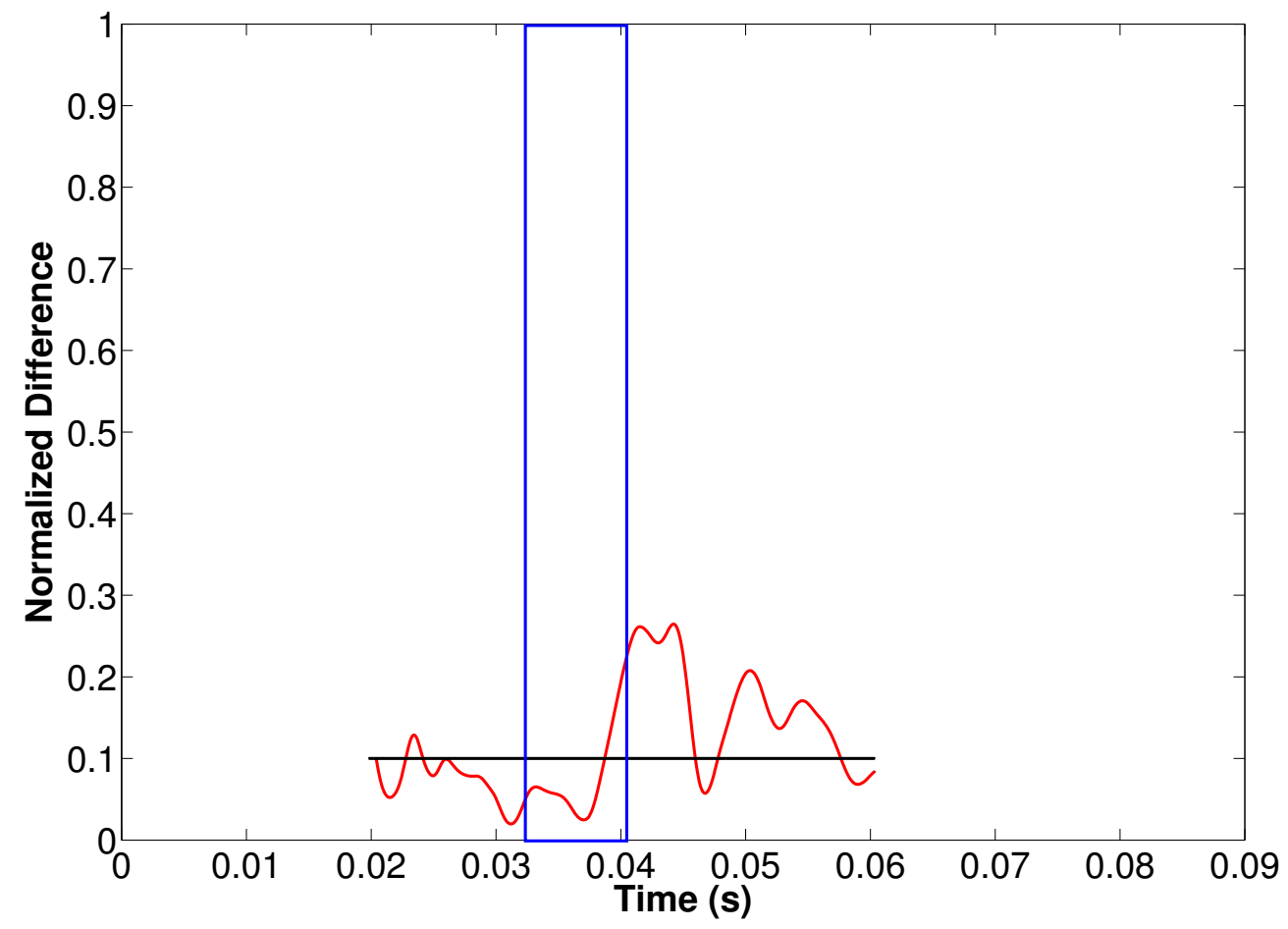

Figure 7.23: Normalized difference plot for the LIF Amplitude Measurement shown in Figure 7.22. At this location the LIF signal amplitude decreases sharply halfway through the perturbation pulse and does not recover until the perturbation ends. 


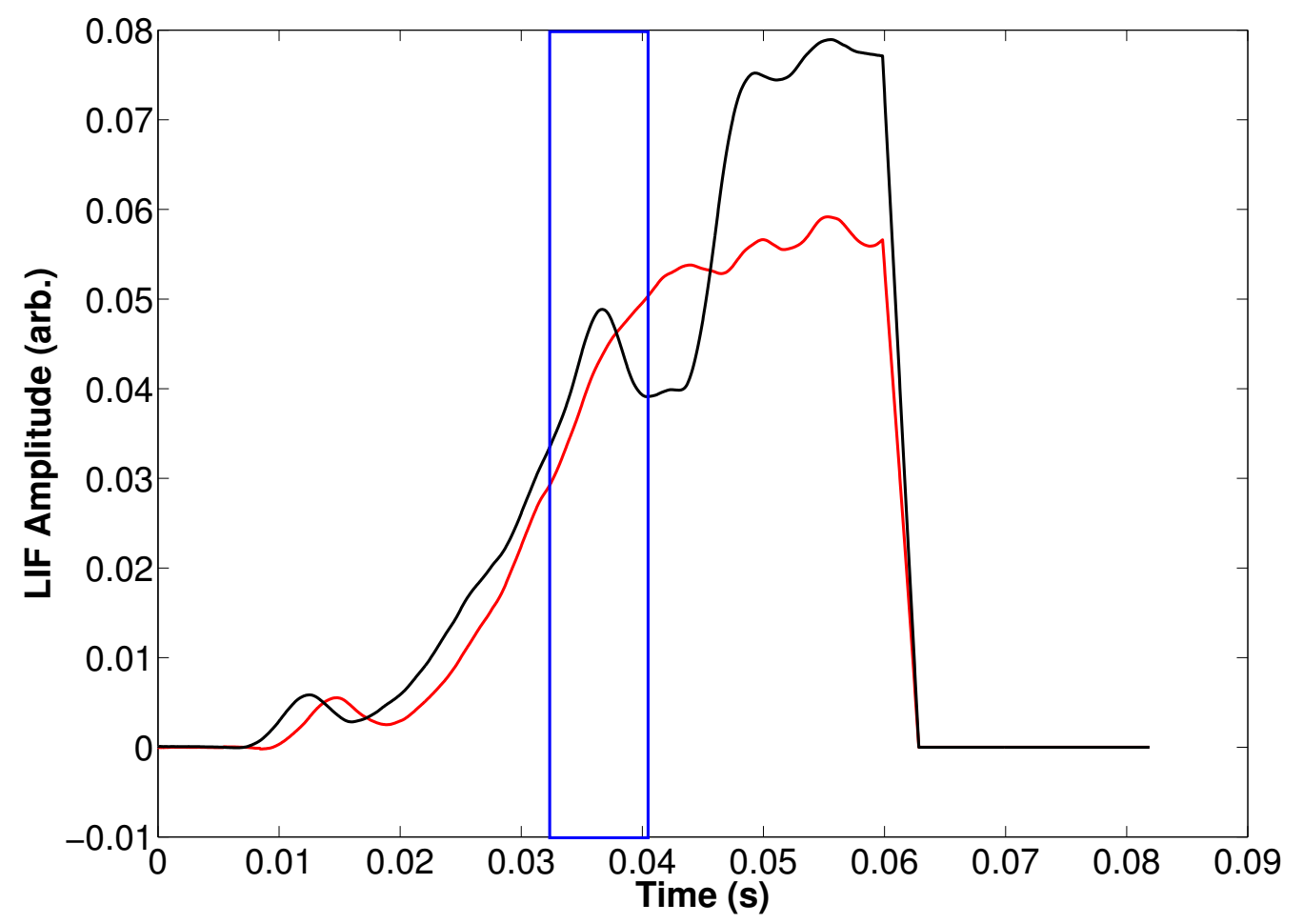

Figure 7.24: LIF signal amplitude during the plasma pulse at $r=0.50 \mathrm{~cm}$, taken on a different day than the data shown in Figure 7.22 to make sure the observations were reproducible. The red line is with no perturbation, the black line is with perturbation, and the blue box is the 8 ms pulse (32.5-40.5 $\mathrm{ms}$ ) when the perturbation is present. As in the previous figure, the perturbation pulse significantly depletes the metastable ion population, which recovers and then exceeds the non-perturbation density after the perturbation ends. 


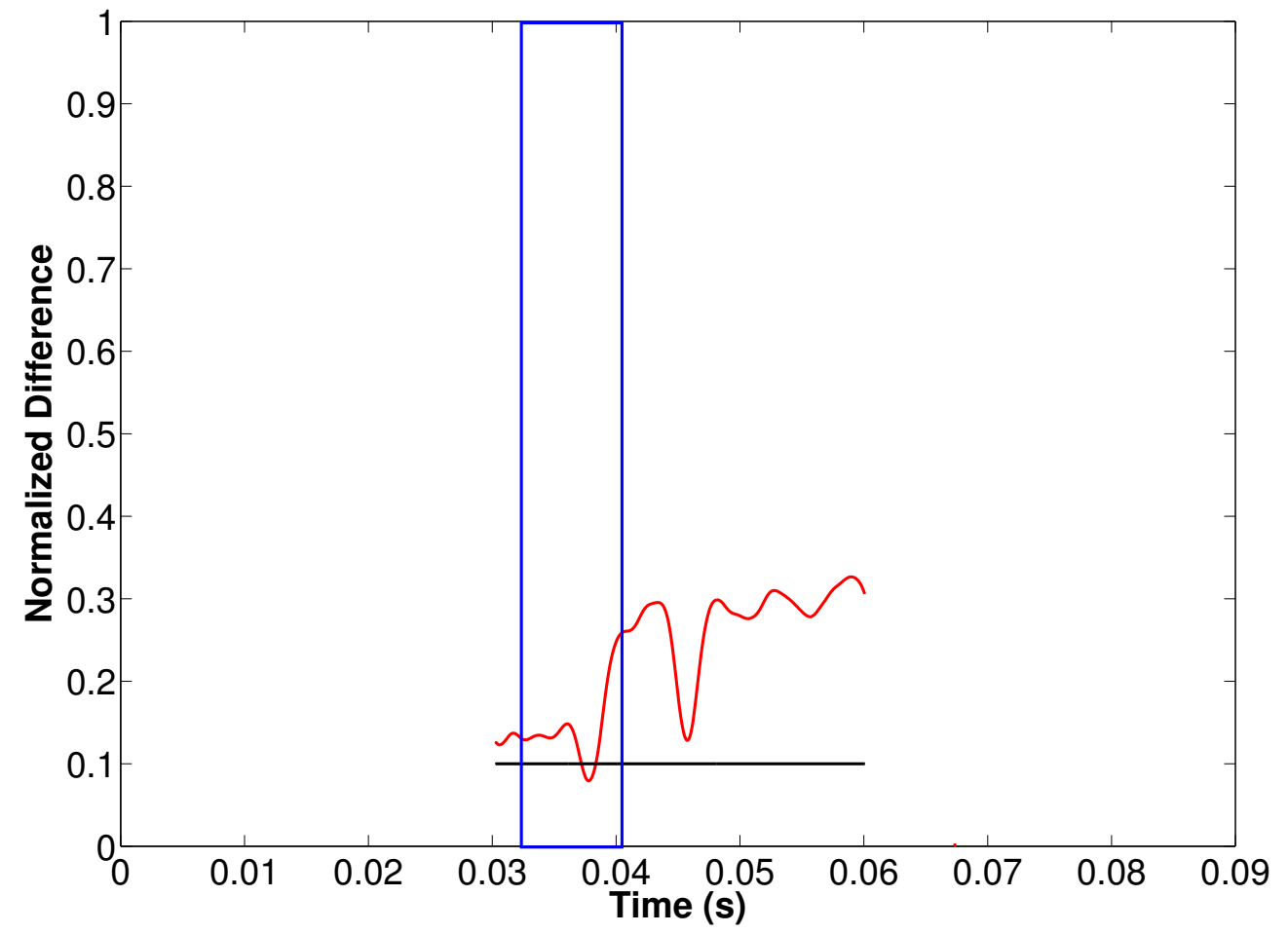

Figure 7.25: Normalized difference plot for the LIF Amplitude Measurement shown in Figure 7.24. Just as seen in Figures 7.22 and 7.23, the LIF signal amplitude decreases sharply halfway through the perturbation pulse and does not recover until the perturbation ends. 


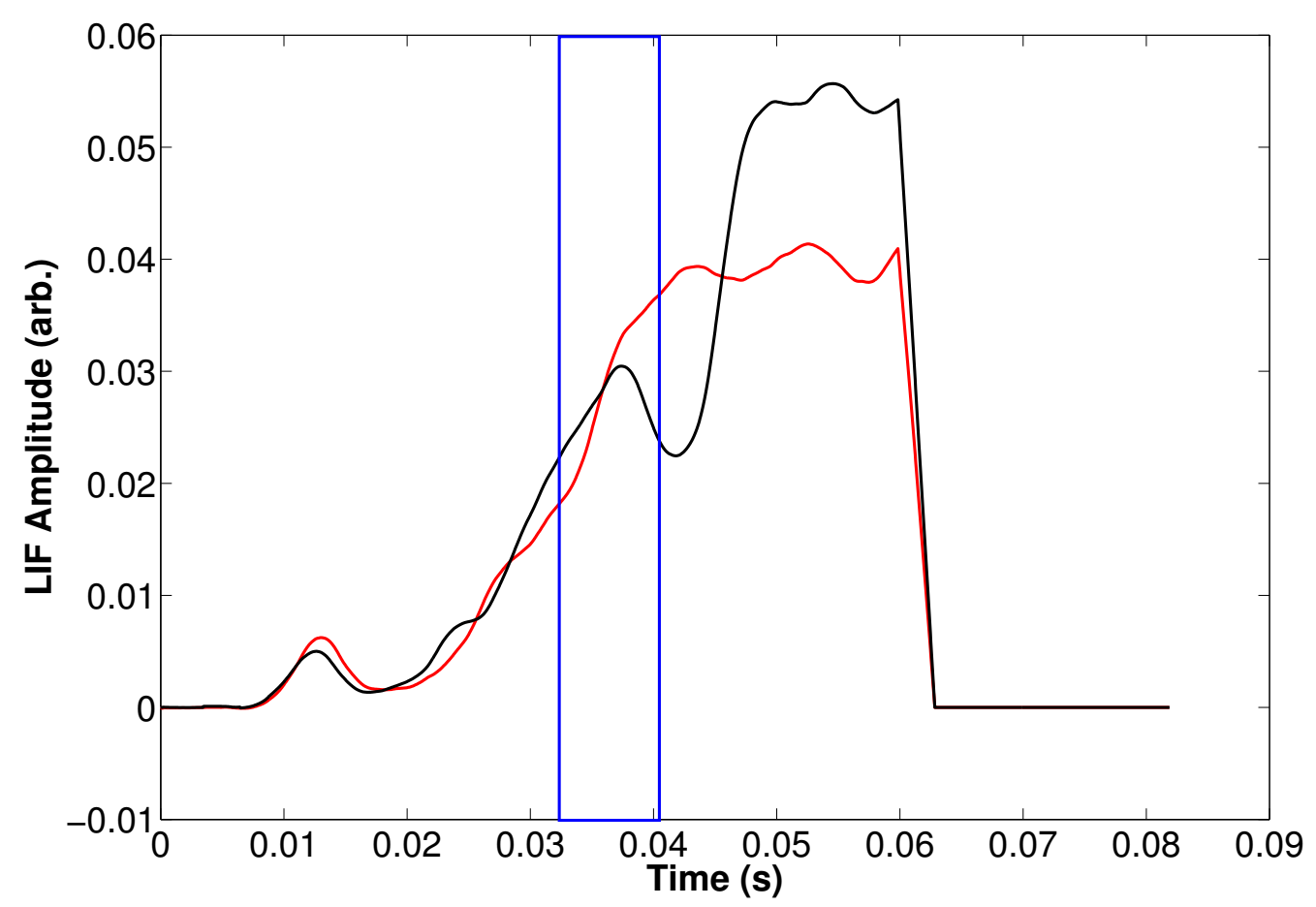

Figure 7.26: LIF signal amplitude during the plasma pulse at $r=0.75 \mathrm{~cm}$. The red line is with no perturbation, the black line is with perturbation, and the blue box is the $8 \mathrm{~ms}$ pulse $(32.5-40.5 \mathrm{~ms})$ when the perturbation is present. At this radial location the perturbation pulse significantly depletes the metastable ion population and, just as seen in Figures 7.22 and 7.24, once the perturbation ends the metastable ion density recovers to exceed the density measured with no perturbation pulse. 


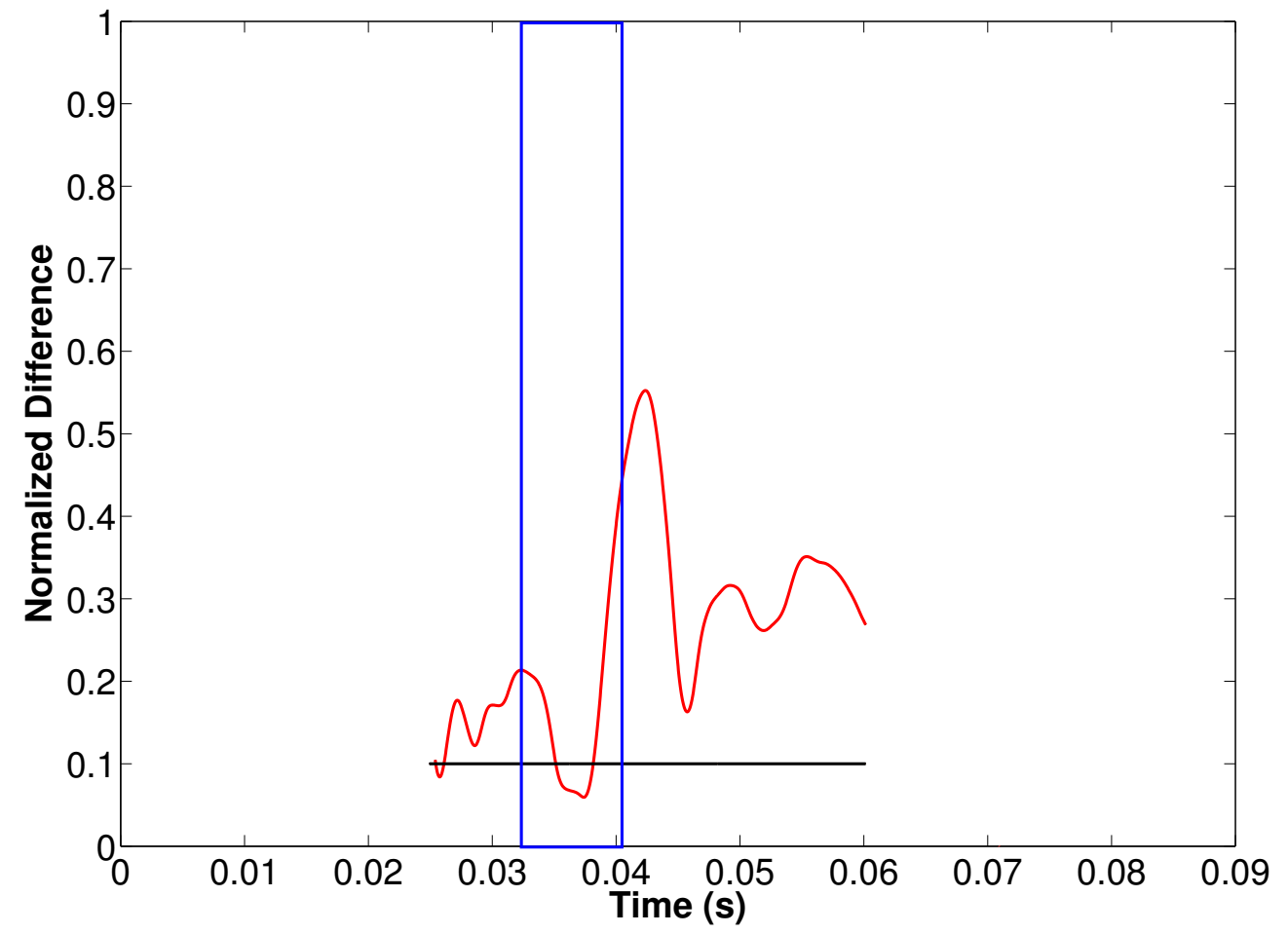

Figure 7.27: Normalized difference plot for the LIF Amplitude Measurement shown in Figure 7.26. As seen at $r=0.5 \mathrm{~cm}$, the perturbation pulse decreases the LIF signal amplitude, which recovers and then exceeds the unperturbed signal amplitude during the last $10 \mathrm{~ms}$ of the discharge. 


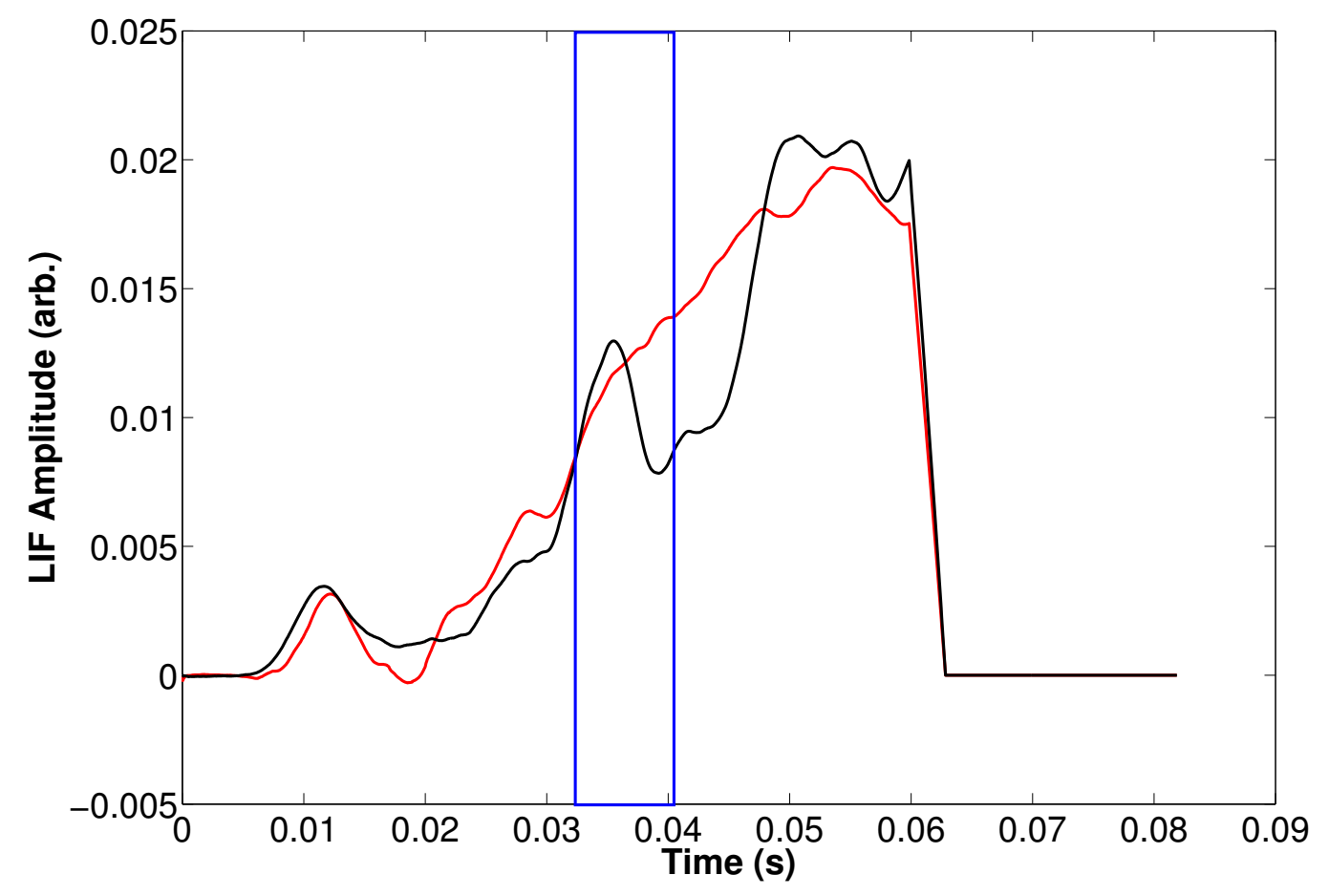

Figure 7.28: LIF signal amplitude during the plasma pulse at $r=1 \mathrm{~cm}$. The red line is with no perturbation, the black line is with perturbation, and the blue box is the $8 \mathrm{~ms}$ pulse $(32.5-40.5 \mathrm{~ms})$ when the perturbation is present. At this radial location the perturbation pulse significantly depletes the metastable ion population, which recovers after the perturbation ends but does not exceed the density observed with no perturbation. 


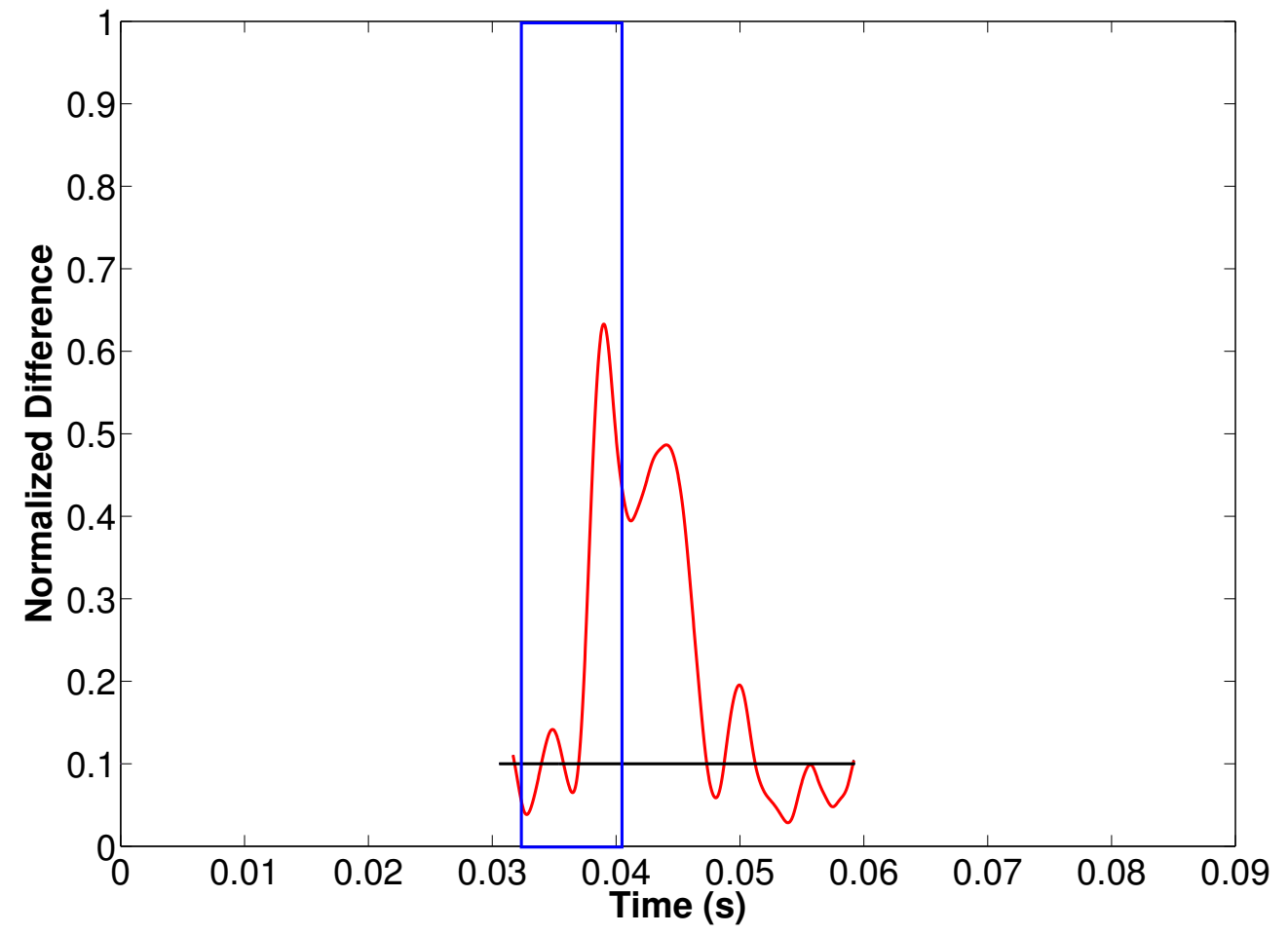

Figure 7.29: Normalized difference plot for the LIF Amplitude Measurement shown in Figure 7.28. The LIF signal amplitude substantially decreases during the perturbation pulse, and only returns to the unperturbed value during the last $10 \mathrm{~ms}$ of the discharge. 
A helpful summary of how the metastable ion density changes across the plasma column in response to the wave perturbation is shown in Figure 7.30. At $r=0 \mathrm{~cm}$ and $r=0.25 \mathrm{~cm}$ the normalized difference in the LIF signal is not statistically significant. At larger radii, however, the difference due to the wave perturbation is significant. 


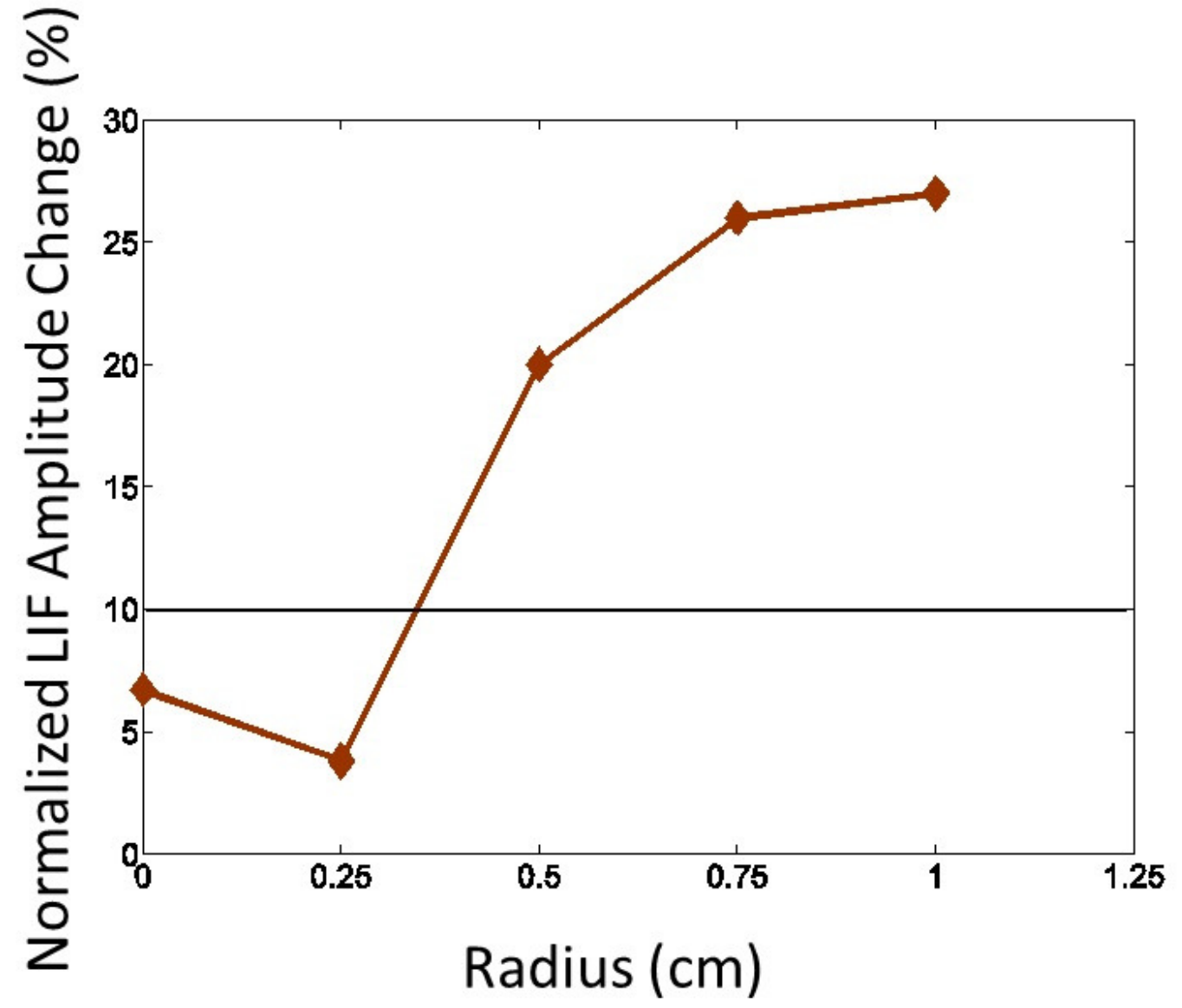

Figure 7.30: The normalized difference in LIF signal amplitude is shown for all radial locations where measurements were made. The difference is not statistically significant at $r=0 \mathrm{~cm}$ and $r=0.25 \mathrm{~cm}$, but it is statistically significant at all larger radii. The $10 \%$ significance threshold is indicated by the black horizontal line. Comparison of this plot with Figure 8.9 clearly shows correlation between metastable density and electron temperature. 


\section{Chapter 8}

\section{Time-Resolved Langmuir Probe Measurements}

\subsection{Langmuir Probe Theory}

The simplest form of a Langmuir probe is a small conductor inserted into a plasma. Analysis of the current collected by the Langmuir probe as a function of externally applied voltage yields information about the electron density, electron temperature, and plasma potential. ${ }^{[92]}$ The main drawback to Langmuir probes, like most in situ diagnostics, is that the presence of the probe perturbs the local plasma environment. This perturbation introduced by the presence of the Langmuir probe makes analysis of the current versus potential plot, called an I-V curve, non-trivial. Detailed discussion and indepth review of Langmuir Probe principles can be found in Hutchinson, ${ }^{933]}$ Hershkowitz, ${ }^{[94]}$ Chen, ${ }^{[95]}$ Schott, ${ }^{[96]}$ Demidov et. al., ${ }^{[97]}$ and Sheridan et. al., ${ }^{[98]}$ as well as others. A brief overview of the theory, design, and data analysis of Langmuir probes is given here.

A typical I-V trace (shown in Fig. 8.1) can be broken into three regions: 


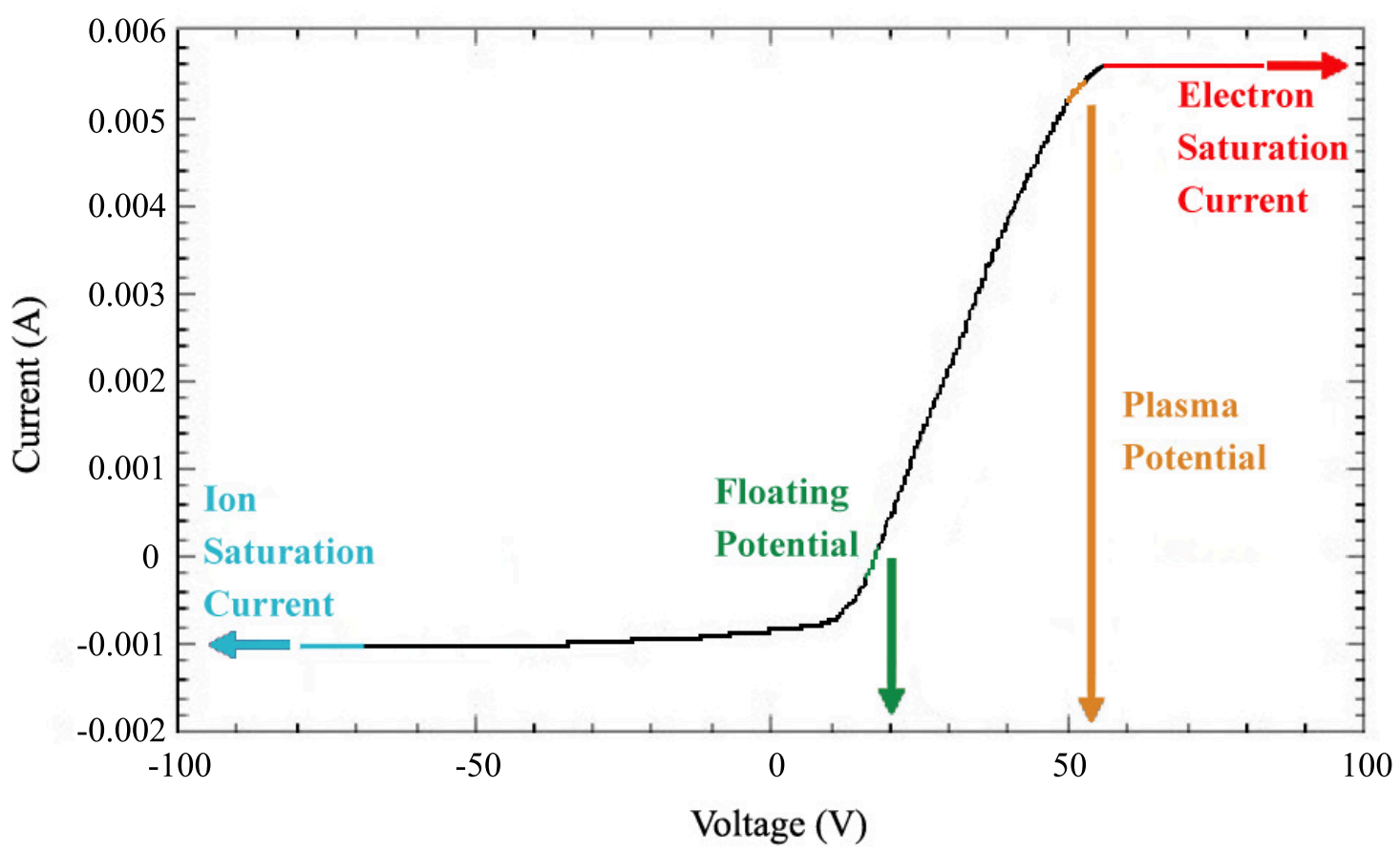

Figure 8.1: An example of an ideal Langmuir probe I-V trace with the ion saturation current, electron saturation current, floating potential and plasma potential indicated. 
the ion saturation region (when the probe is biased sufficiently negative to repel all electrons), the transition region (when the probe collects both ions and electrons), and the electron saturation region (when the probe is biased sufficiently positive to repel all ions). Since electrons are much more mobile than ions, the magnitude of the electron saturation current is much greater than that of the ion saturation current. In a quasi-neutral plasma, a Langmuir probe charges slightly negative since the lighter weight and higher velocities of electrons, compared to ions, means they have a higher flux than the ions. The potential at which the probe draws no net current is called the floating potential $V_{f}$ while the potential where electron saturation starts, the electric potential of the plasma, is called the plasma potential $V_{p}$. The floating potential is related to both the electron temperature and the plasma potential.

Assuming the particle distributions are Maxwellian, the plasma is collisionless, and there is no magnetic field, the current near the floating potential can be written as

$$
I\left(V_{0}-V_{p}\right)=n_{e} e A_{p}\left(\frac{k_{B} T_{e}}{m_{i}}\right)^{1 / 2}\left[\left(\frac{1}{2}\right)\left(\frac{2 m_{i}}{\pi m_{e}}\right) \mathrm{e}^{\left(\frac{e\left(V_{0}-V_{p}\right)}{k_{B} T_{e}}\right)}-\left(\frac{A_{s}}{A_{p}}\right) \mathrm{e}^{\left(-\frac{1}{2}\right)}\right]
$$


where $V_{0}$ is the externally applied voltage, $V_{p}$ is the plasma potential, $n_{e}$ is the plasma density, $e$ is the elementary charge, $T_{e}$ is the electron temperature, $m_{i}$ is the ion mass, $m_{e}$ is the electron mass, $A_{s}$ is the surface area of the sheath around the probe, and $A_{p}$ is the surface area of the probe. ${ }^{[3]}$ If the probe size is much larger than the thickness of the surrounding sheath, $A_{s} / A_{p} \approx 1$ is a good approximation. The sheath is a region of varying potential formed as the charges in the plasma move to screen out the potential applied to the probe. ${ }^{[99]}$ As $V_{0}$ becomes more negative, the first term inside the brackets of Eqn. 8.1 becomes negligible. Keeping only the second term inside the brackets yields

$$
I_{s i}=-e J_{i}=-0.61 e n_{e} A_{p} \sqrt{T_{e} / m_{i}}
$$

where $I_{s i}$ is the ion saturation current and $J_{i}$ is the ion current density in an unmagnetized plasma. In order to solve Eqn. 8.2, the electron temperature and plasma density are needed. Electron temperature is obtained by taking the derivative of Eqn. 8.1 with respect to the voltage $V=V_{0}=V_{p}$, which 
yields

$$
\frac{d I(V)}{d V} \cong \frac{e}{T_{e}}\left(I-I_{s i}\right)+\frac{d I_{s i}}{d V}
$$

The second term can be neglected because $d I_{s i} / d V \gg d I(V) / d V$ in the saturation regime. Solving Eqn. 8.3 for $T_{e}$ yields

$$
T_{e}=\frac{e\left[I(V)-I_{s i}\right]}{\frac{d I(V)}{d V}}
$$

By performing a linear fit to the semi-logarithmic plot of $\ln \left(I-I_{s i}\right)$ versus $V$ and taking the inverse of the slope of the fit, the electron temperature may be obtained. Then by putting the calculated electron temperature and measured ion saturation current into Eqn. 8.2, the plasma density can also be determined.

Experimental constraints, especially in high-density plasmas such as helicon sources, produce I-V traces that deviate from the ideal. In a helicon source a cylindrical Langmuir probe never achieves true electron saturation because the sheath around the probe continues to expand, and therefore collect more current, as the voltage is increased. ${ }^{[00]}$ Without true electron saturation, the plasma potential cannot be directly measured and must instead 
be estimated. In a plasma where $T_{i}<T_{e}$, a helpful relationship between the plasma potential and floating potential can be used.

The ion current floating potential is

$$
j_{i}=\frac{1}{4} n e \sqrt{\frac{8 k_{B} T_{e}}{\pi m_{i}}}
$$

while the electron current at the floating potential is

$$
j_{e}=\frac{1}{4} n e \sqrt{\frac{8 k_{B} T_{e}}{\pi m_{i}}} \exp \left(\frac{e\left(V_{f}-V_{p}\right)}{k_{B} T_{e}}\right) .
$$

Recalling that at the floating potential, the net current is zero, it must be true that $j_{i}=j_{e}$. After carefully manipulating the terms above, we get a relation for the plasma potential in terms of the floating potential: ${ }^{[101]}$

$$
V_{p}=V_{f}+\frac{k_{B} T_{e}}{2 e} \ln \left(\frac{T_{e} m_{i}}{T_{e} m_{e}}\right)=V_{f}+\frac{k_{B} T_{e}}{2 e} \ln \left(\frac{m_{i}}{m_{e}}\right) .
$$

For argon ions, $m_{i}=40 m_{p}$, where $m_{p}$ is the proton mass, which reduces Eqn. 8.7 to

$$
V_{p}=V_{f}+5.6 T_{e} .
$$


Looking at Eqn. 8.8, we see that for argon, the difference between the plasma potential and the floating potential is 5.6 times the electron temperature and that the slope $d I / d V_{\text {applied }}$ can be used as a reasonable approximation for $d I / d V$ in order to calculate the electron temperature.

The discussion of Langmuir probe theory to this point has been for unmagnetized plasma. In the presence of a magnetic field, ions and electrons gyrate around the field lines, limiting cross-field transport and thus decreasing the particle flux to the probe. The significance of magnetic field effects is determined by the ratio of the gyroradius to the characteristic dimension of the probe. If this ratio is much smaller than one (i.e., if the probe is much larger than the gyroradius) for a given species, that species will be significantly impeded from interacting with the Langmuir probe. To account for cross-field transport and collisions, Eqns. 8.1- 8.4 must be modified. ${ }^{[93]}$ For an ion temperature of $0.3 \mathrm{eV}$ and a magnetic field strength of $1 \mathrm{kG}$ in HELIX, the gyroradius of an ion is $\cong 3.5 \mathrm{~mm}$, which is comparable to the $2 \mathrm{~mm}$ Langmuir probe tip length and larger than the $0.5 \mathrm{~mm}$ tip diameter. Including the magnetic field effects on the ions, Hutchinson ${ }^{[93]}$ showed that 
Eqn. 8.2 must be adjusted:

$$
I_{s i}=-e J_{i}=-0.49 e n_{e} A_{p} \sqrt{T_{e} / m_{i}} .
$$

In addition to the presence of the magnetic field in HELIX, we must also consider the effects strong RF fields have on Langmuir probe measurements. RF fields in the helicon source can accelerate particles both toward and away from the probe. The sloshing of the electrons back and forth in the sheath introduces error in the measurements of the floating potential and broadens the electron distribution function. ${ }^{[61]}$ To minimize these errors in measurement, a Langmuir probe must be RF compensated. Sudit and Chen ${ }^{[102]}$ developed an RF compensation method with the addition of a floating electrode and small capacitor. The floating electrode is exposed to the plasma potential fluctuations and connected to the Langmuir probe tip through the capacitor. This forces the Langmuir probe tip to follow the potential oscillations in the plasma and thereby reduces the sheath impedance. In the work presented here, the Langmuir probe has a similar floating electrode, but that electrode is not exposed directly to the plasma. A set of RF chokes (small inductors) are also connected between the Langmuir probe tip and the external voltage 


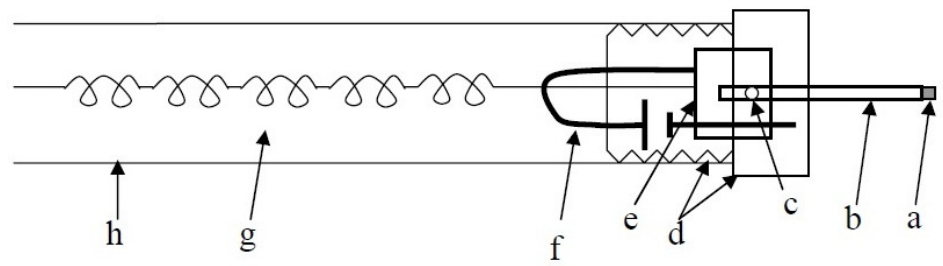

Figure 8.2: Schematic representation of the RF-compensated Langmuir probe used in this work. Key features include the graphite tip (a), the insulating alumina shaft (b), the set screw that holds the alumina in place (c), the threaded boron nitride cap (d), the conducting copper base (e), the capacitor (f), the chain of rf chokes (g), and the stainless steel probe shaft (h). Figure obtained from Keesee. ${ }^{[88]}$

source. These chokes increase the impedance of the current measurement circuit at the RF frequency which generates the plasma.

A schematic of the Langmuir probe used in this work is shown in Fig. 8.2. The probe tip is made of a graphite rod (commercially available for mechanical pencils) with a $0.5 \mathrm{~mm}$ diameter. The graphite is mounted into a conducting copper base and surrounded by a $0.6 \mathrm{~mm}$ insulating alumina shaft in such a way that only $2 \mathrm{~mm}$ of the very end is exposed to the plasma. The copper base is connected to a $10 \mathrm{nF}$ capacitor and a chain of RF chokes (inductors) chosen to block a specific range of RF frequencies. From the copper base and moving down the probe shaft, the inductor chain is: $26,53,26$, 13.2, and 6.8 MHz. ${ }^{[103]}$ The end of the last RF choke inductor is soldered to a shielded coaxial wire which is attached to a BNC vacuum feedthrough at 
the far end of the stainless steel probe shaft. In these experiments, the bias voltage of the Langmuir probe was supplied by a Keithley 2400 SourceMeter which was controlled via a GPIB interface by custom software written in LabWindows $^{\mathrm{TM}}$. All of the Langmuir probe measurements presented here have a time resolution of $3 \mathrm{~ms}$; this is the limit of the digitization rate of the Keithley 2400 SourceMeter.

\subsection{Time-Resolved Langmuir Probe Measurements}

Figures 8.3- 8.5 show time-resolved electron density and electron temperature measurements at six different radial locations across the plasma column. While the electron density does not appear to change in the presence of the wave perturbation, except possibly at the very center of the plasma where the density is greatest (see Figure 8.6), the electron temperature does change in response to the wave perturbation (see Figures 8.4, 8.7, and 8.9). This indicates that the low-frequency perturbation is driving wave-particle interactions in the plasma which heat the electrons. By looking at how the temperature change due to the wave perturbation compares to the steady state electron temperature, it is possible to get an idea where in the plasma (i.e., in relation to the local density and density gradient) the wave interacts 
most strongly. Figures 8.5 and 8.8 show the relative electron temperature change over time in the plasma pulse for all six radial locations, while Figure 8.9 shows the relative electron temperature change versus radius for two sets of radial measurements on opposite sides of the plasma column.

The first set of electron density and temperature Langmuir probe measurements (Figures 8.3- 8.5) were taken $45 \mathrm{~cm}$ downstream from the wavelaunching antenna and on the same side of the chamber. The first set of density measurements, figure 8.3, shows no change in the electron density due to the wave perturbation at any radial location. Notice that the electron density is lowest at $r=0.75 \mathrm{~cm}$, which is where the center of the driven current channel is located. Figure 8.4 indicates electron heating in response to the wave perturbation at all radial locations. Normalizing the change in electron temperature to the steady-state electron temperature (see Figures 8.5 and 8.9) shows that the relative heating changes with radius. Figure 8.9 shows that for this set of measurements, the largest relative electron temperature change is at $r=1 \mathrm{~cm}$, with a slightly smaller change at $r=0.75 \mathrm{~cm}$ and a still smaller change at $r=0.5 \mathrm{~cm}$. At $r=1.25 \mathrm{~cm}$, the relative electron temperature change is half that seen at $r=1 \mathrm{~cm}$, while at $r=0 \mathrm{~cm}$ and $r=0.25$ $\mathrm{cm}$, the relative change is less than a third of the maximum. Density gradient 
effects could be important, since the strongest heating is observed between $r=0.5 \mathrm{~cm}$ and $r=1.25 \mathrm{~cm}$; but it is also possible the temperature change is simply due to Ohmic heating, since this set of radial measurements passed through the perturbed current channel.

To rule out Ohmic heating as the source of electron temperature change, a second set of Langmuir probe measurements was taken at the same axial location on the opposite of the chamber. The second set of Langmuir probe density measurements (Figure 8.6) indicate a possible density increase at the very center of the plasma, but nowhere else. Figures 8.7, 8.8, and 8.9 show the electrons are heated in response to the wave perturbation outside of the current channel. Figure 8.9 shows the relative electron heating is still peaked at $r=1 \mathrm{~cm}$, and the behavior at $r=0.25 \mathrm{~cm}, r=0.5 \mathrm{~cm}$, and $r=1.25$ $\mathrm{cm}$ is consistent with the observations in the first set of measurements. The relative electron heating at $r=0.75 \mathrm{~cm}$ is lower at the second location, which could be due to some Ohmic heating in the first set of measurements. The relative electron heating at $r=0 \mathrm{~cm}$ is noticeably higher in the second set of measurements than in the first. This relative heating difference at $r=0$ $\mathrm{cm}$, like the possible density change shown in Figure 8.6, between the two measurements could be due to physical "shadowing" of the Langmuir probe 
by the wave-launching antenna during the first set of measurements. Electron heating in this second set of measurements indicates heating throughout symmetric annuli around the plasma core, not just Ohmic heating in the driven current channel.

Comparing the electron temperature observations in this work to other electron heating observations in laboratory Alfvén wave studies is important. Observations of Alfvén wave propagation in LAPD driven by a quadrupole antenna have included Langmuir probe measurements of the electron temperature. As shown in Figure 8.10, the observed electron heating in LAPD is confined to the current channels driven by the quadrupole antenna. In other words, Ohmic heating of the electrons is observed in LAPD Alfvén waves studies, but not heating due to wave-particle interactions throughout the plasma. 


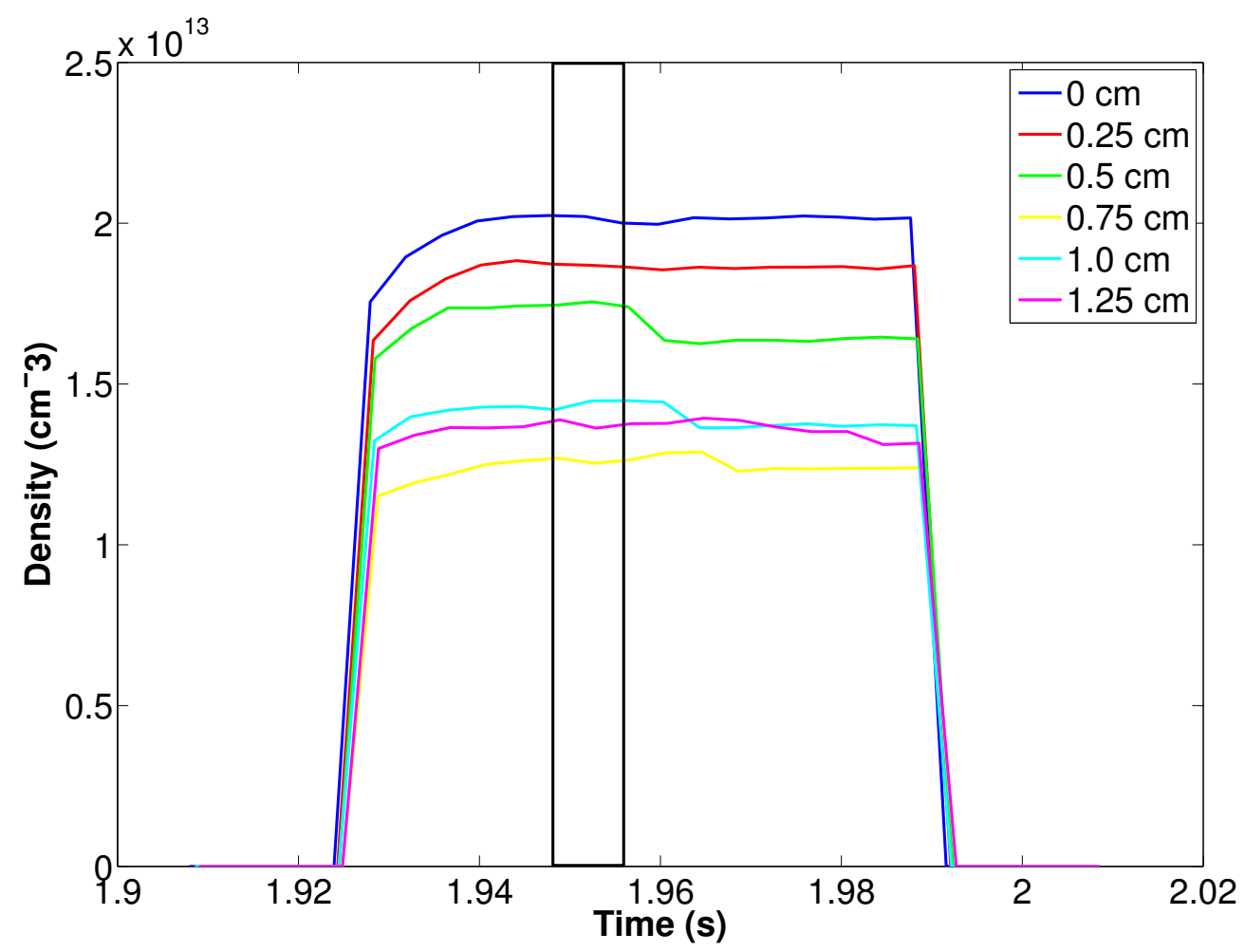

Figure 8.3: Time-resolved electron density during the plasma pulse. The different colored traces are the six different radii where time-resolved Langmuir probe measurements were taken and the black box indicates the $8 \mathrm{~ms}$ when the wave perturbation was driven. Notice that the density does not appear to change in response to the wave perturbation. The center of the driven current channel is at $r=0.75 \mathrm{~cm}$. 


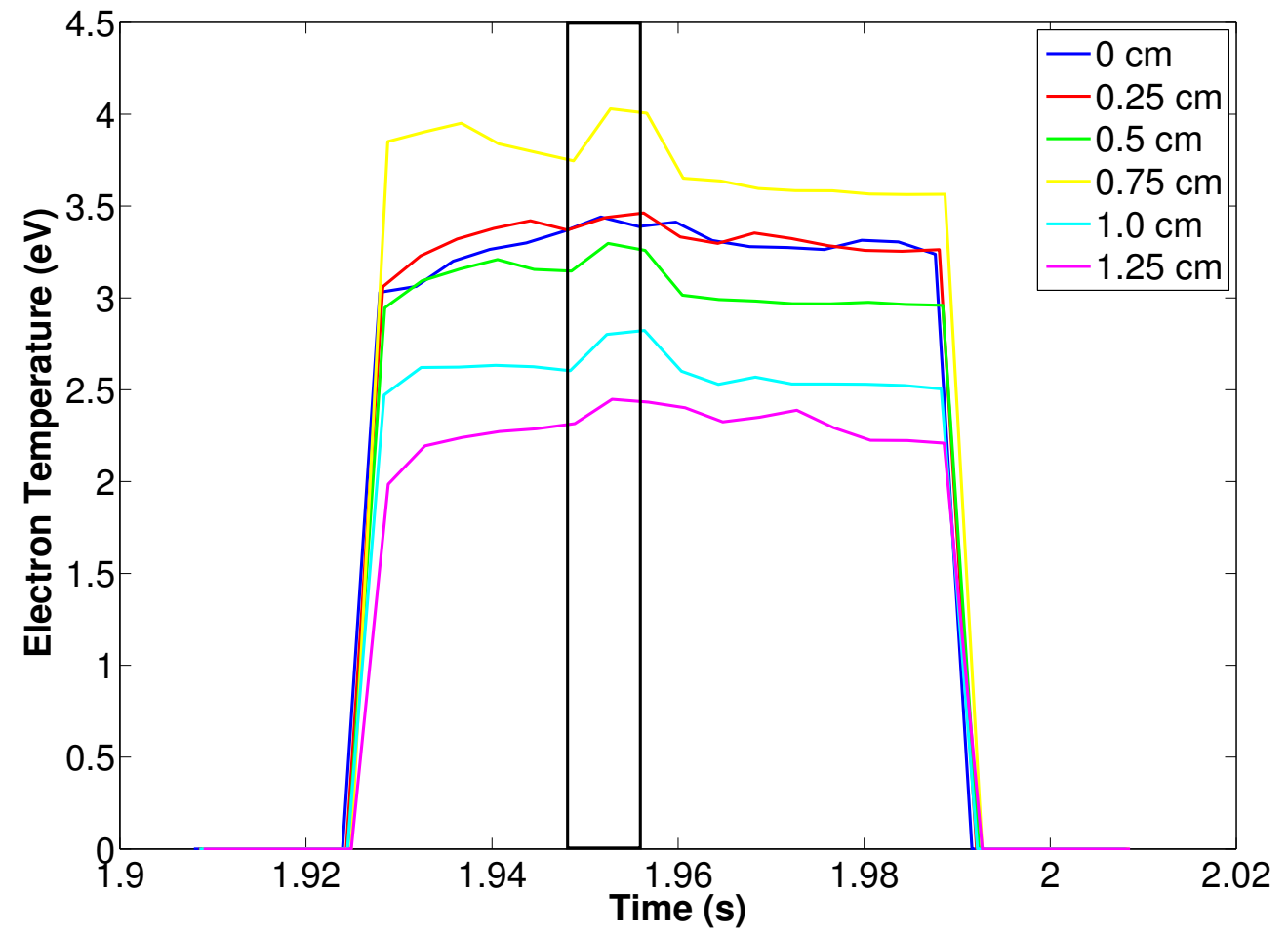

Figure 8.4: Time-resolved electron temperature for six different radial locations during the plasma pulse. The electron temperature appears to change in response to the Alfvén wave perturbation (indicated by the black box in the figure). Notice that the electron temperature is largest at $r=0.75 \mathrm{~cm}$, which is the center of the driven current channel. 


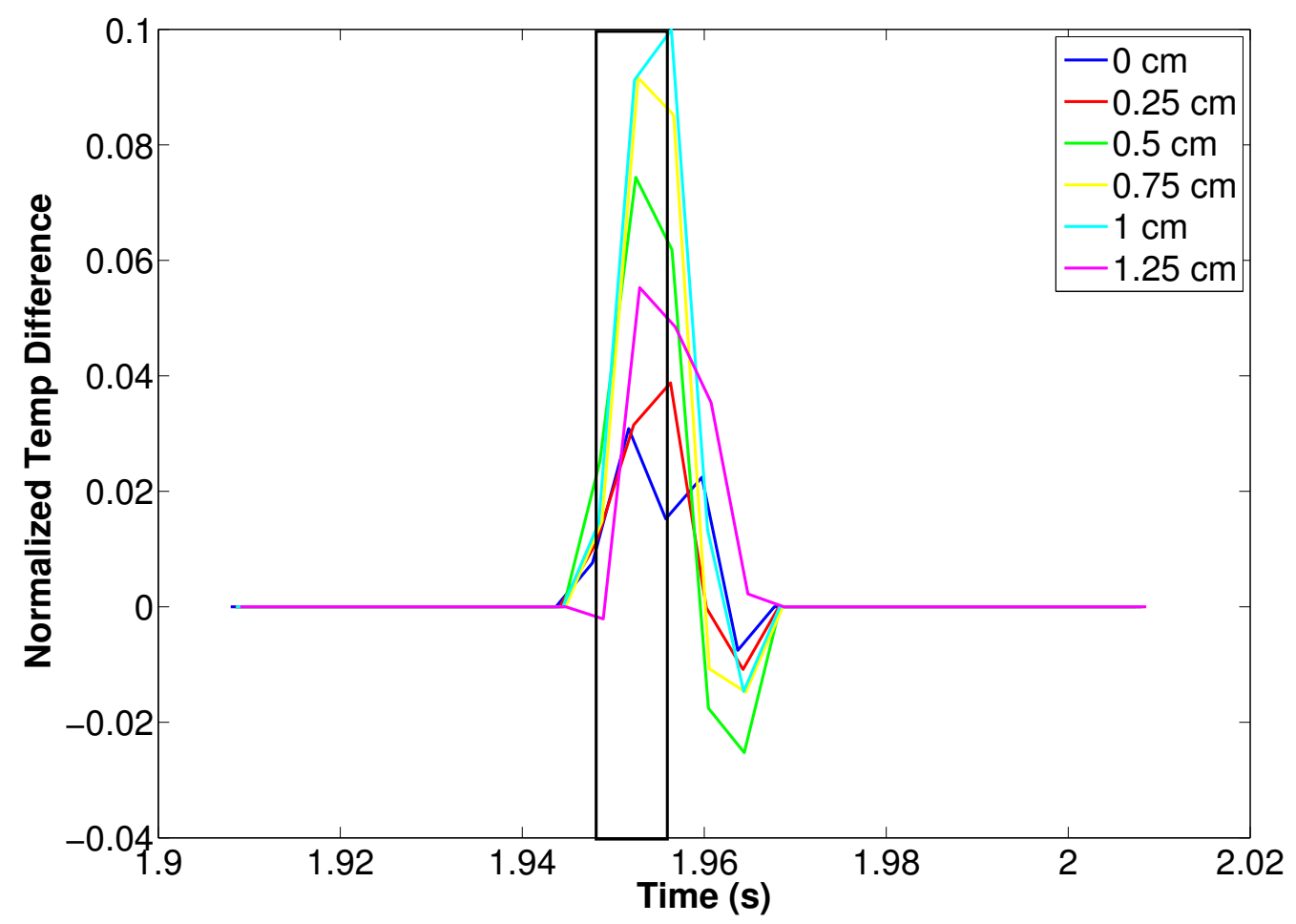

Figure 8.5: When the change in electron temperature due to the wave perturbation is normalized to the steady-state electron temperature (the electron temperature without the wave perturbation) it can be clearly seen that the largest change in temperature due to the wave interaction is at $r=1 \mathrm{~cm}$ (see Figure 8.9). Again, the black box indicates the $8 \mathrm{~ms}$ of the perturbation pulse. 


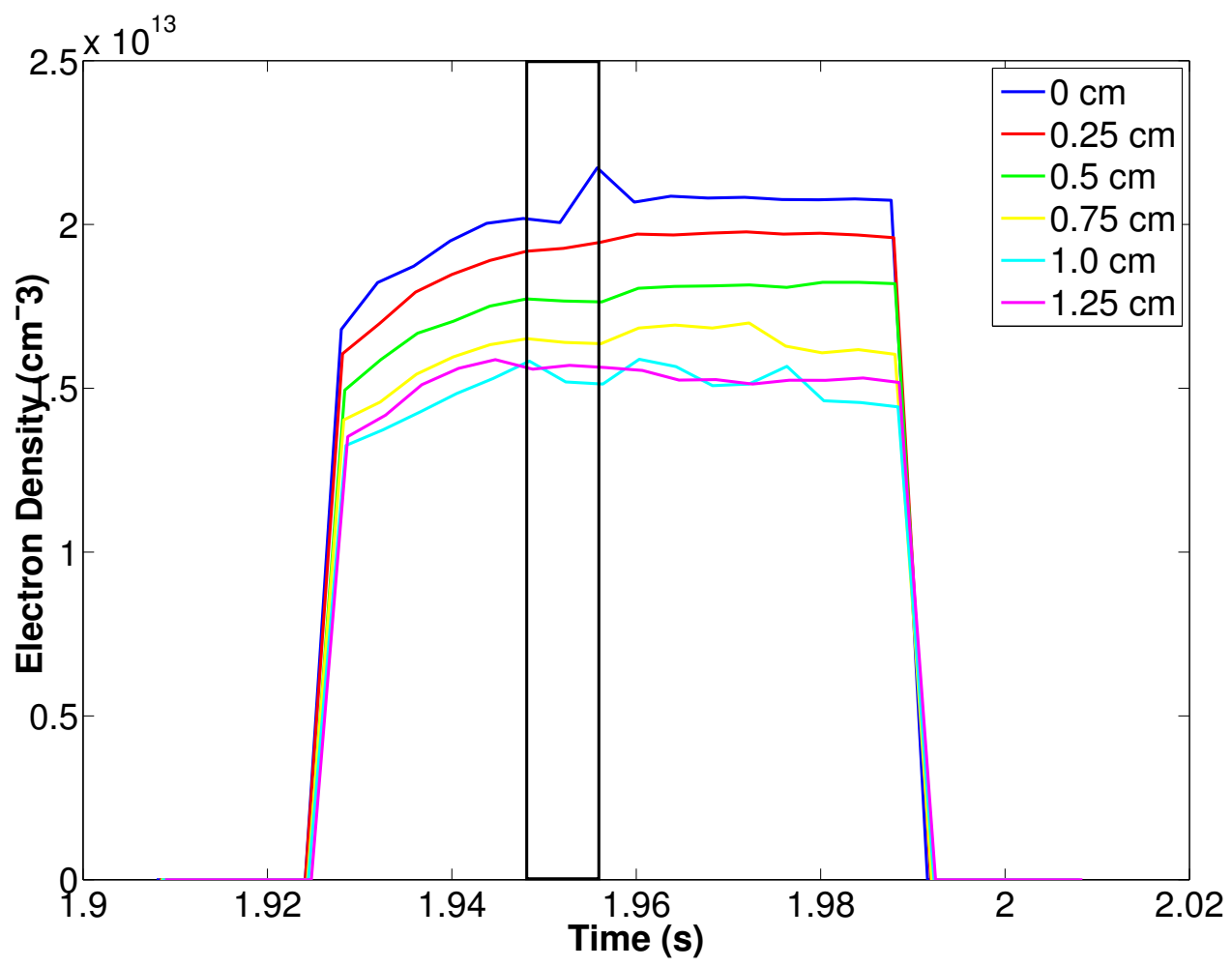

Figure 8.6: Time-resolved electron density during the plasma pulse. Notice that at $r=0 \mathrm{~cm}$, the density appears to increase due to the perturbation, unlike in the first set of measurements (see Figure 8.3). The different colored traces are the six different radii where time-resolved Langmuir probe measurements were taken and the black box indicates the $8 \mathrm{~ms}$ when the wave perturbation was active. 


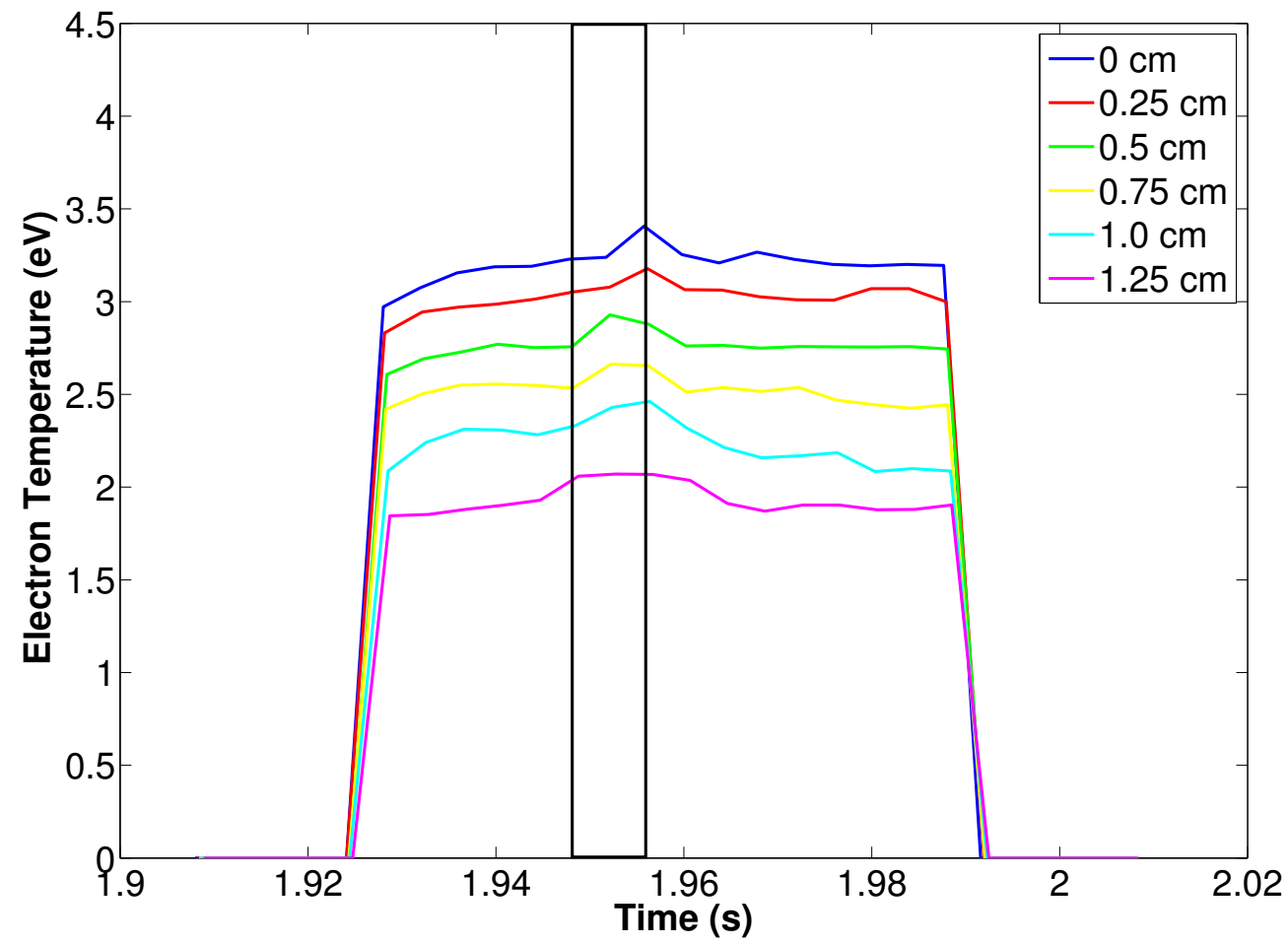

Figure 8.7: Time-resolved electron temperature for six different radial locations during the plasma pulse. The electron temperature clearly changes in response to the Alfvén wave perturbation, just as in the first set of measurements (see Figure 8.4. 


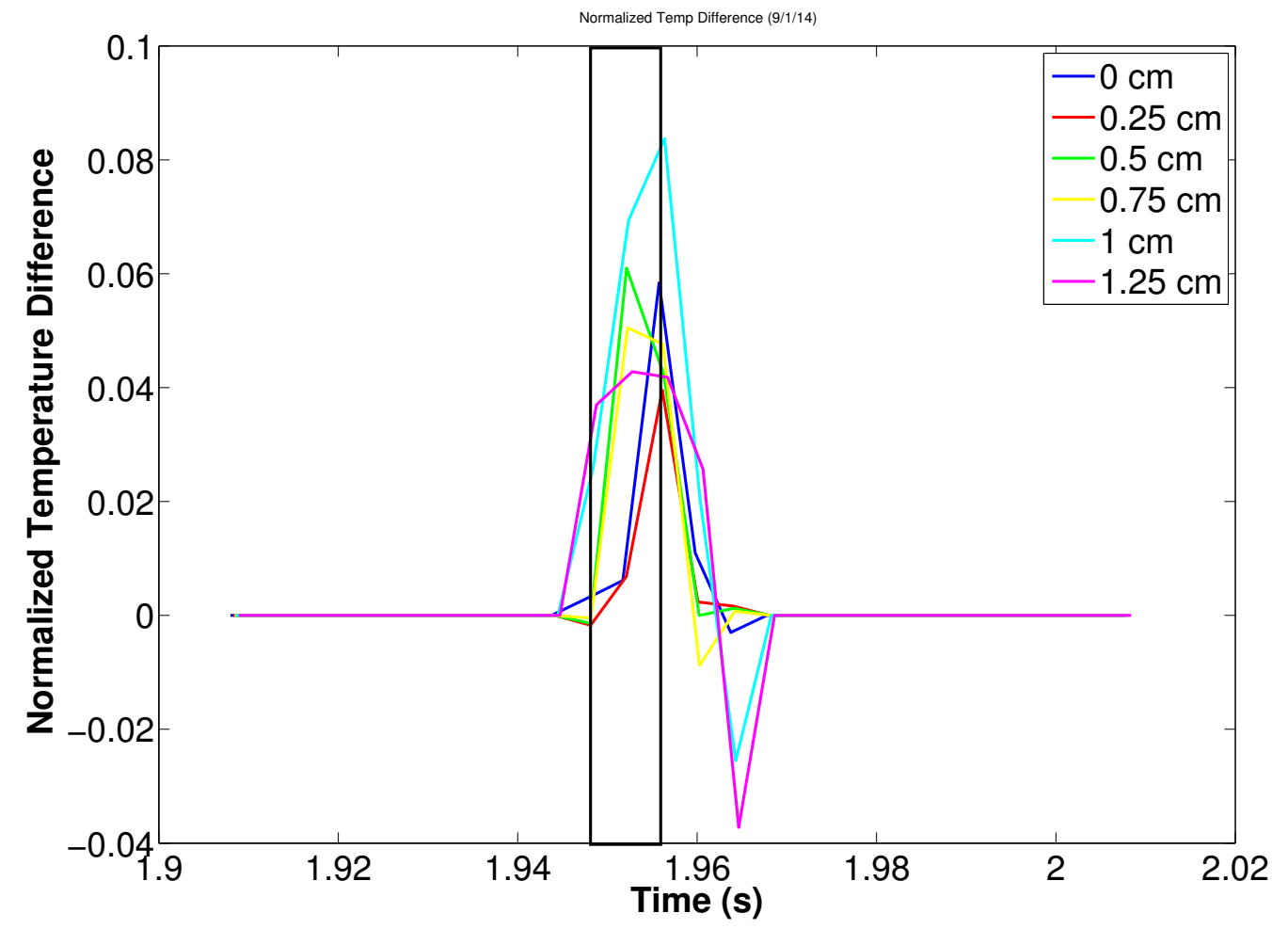

Figure 8.8: The change in electron temperature due to the wave perturbation normalized to the steady-state electron temperature shows that the wave couples most strongly to the electrons at $r=1 \mathrm{~cm}$ (see Figure 8.9 for better visualization). This indicates that the effects of the density gradient play an important aspect in the propagation of the wave. 


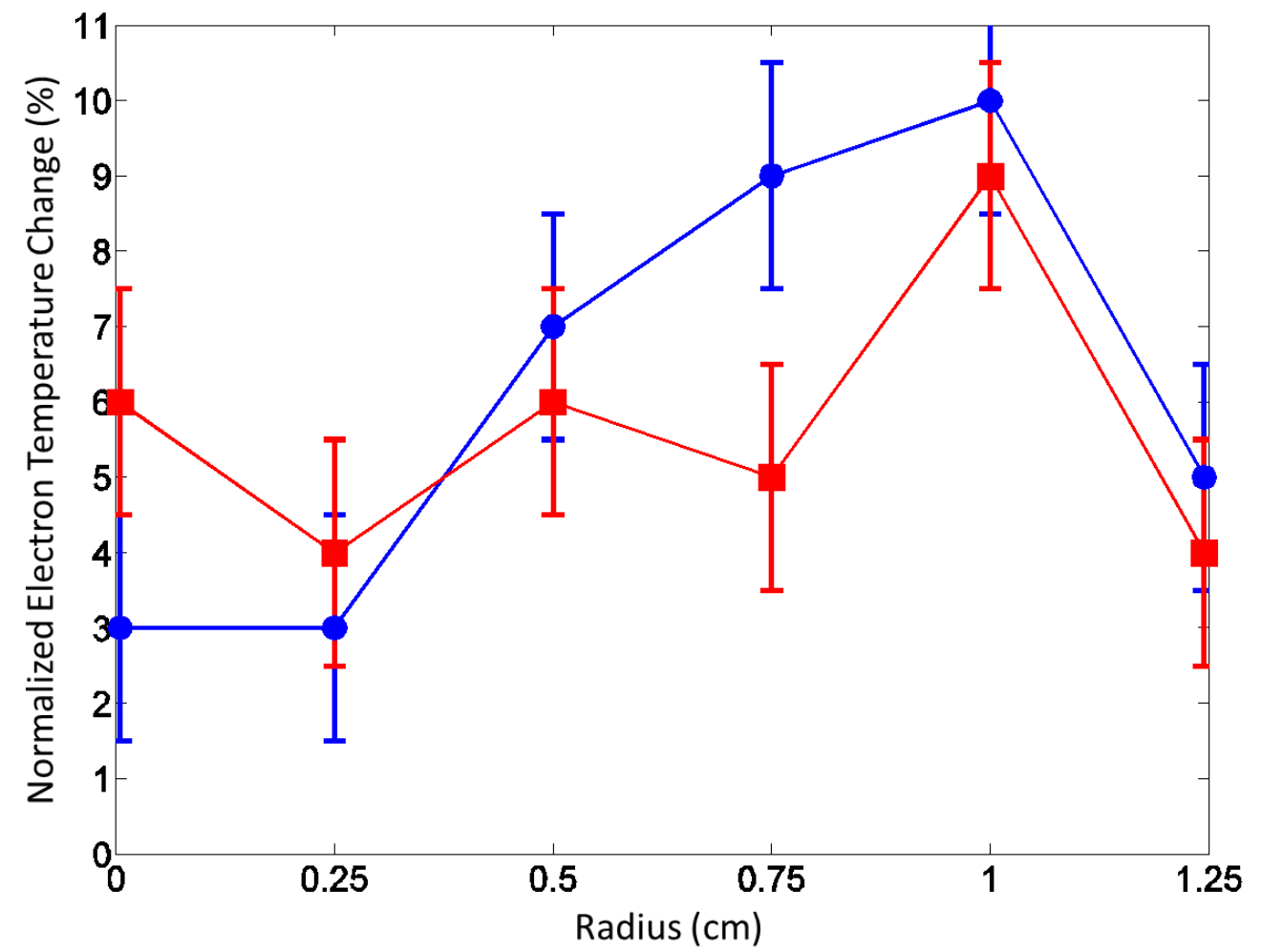

Figure 8.9: The relative electron temperature change versus radius. The red squares are the first set of measurements, which passed through the driven current channel (centered at $r=0.75 \mathrm{~cm}$ ). The blue circles are the second set of measurementstaken on the opposite side of the plasma column. 

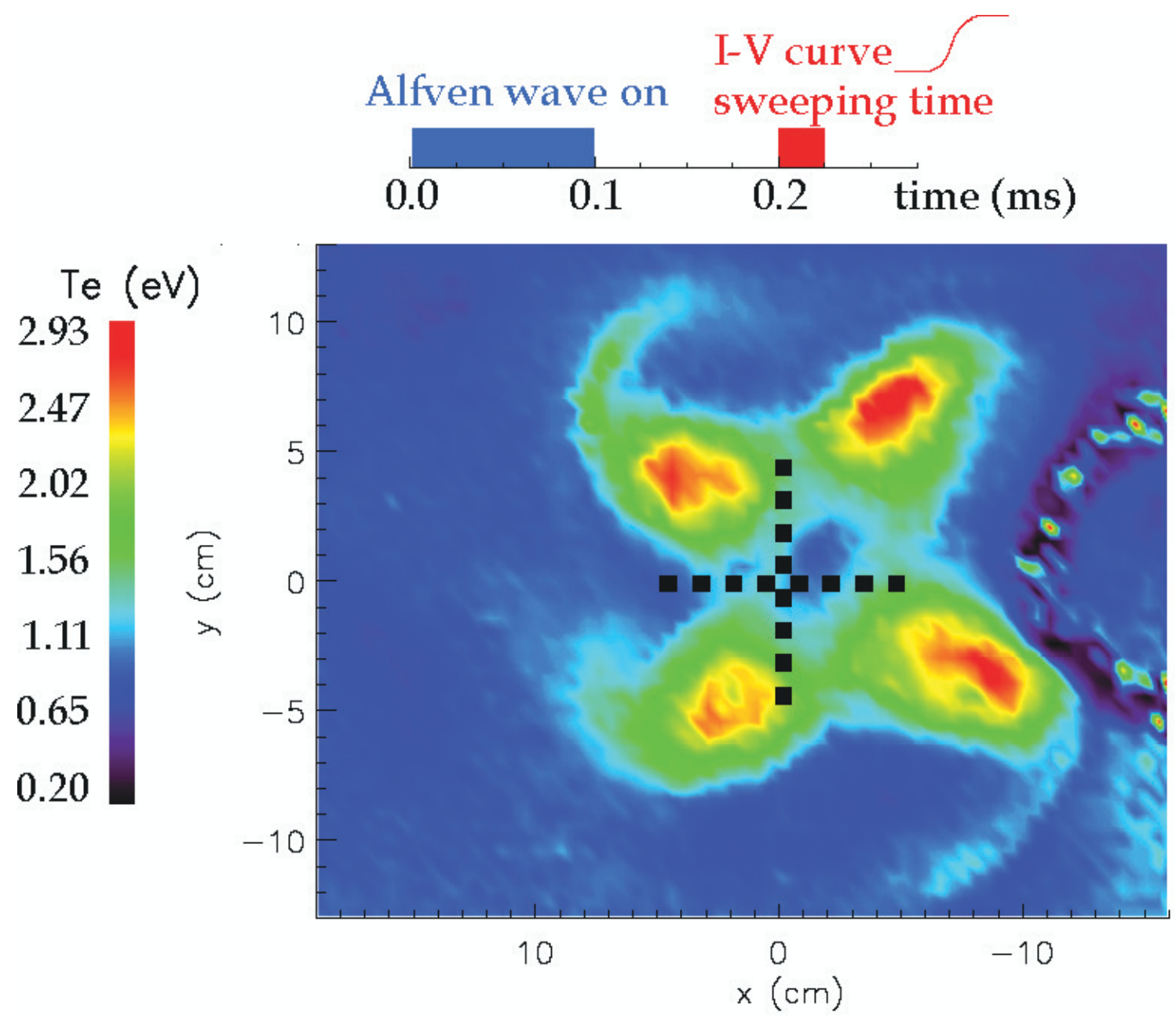

Figure 8.10: Electron heating observations during Alfvén wave propagation in LAPD. The localization of the hot spots to the quadrapole pattern of the wave-launching antenna (antenna location marked by the dotted black lines) indicates Ohmic heating of the electrons. ${ }^{[104]}$ 


\section{Chapter 9}

\section{Summary and Conclusions}

The scope of this work was to investigate wave-particle interactions in a helicon source with a density gradient in response to a driven low-frequency magnetic perturbation. Time-resolved Laser-Induced Fluorescence (LIF) measurements hint at $0.25-0.5 \mathrm{eV}$ ion heating, $\sim 20 \%$, at the center of the plasma, although no heating is indicated at larger radii (where the density drops). These same LIF measurements show a depletion in argon metastable density ranging from $20 \%-50 \%$, depending on radius, during the perturbation pulse. At the edge of the high-density core (around $r=1 \mathrm{~cm}$ ) the signal-tonoise ratio is very low, making determination of possible ion heating much more difficult. Wavelet analysis of the magnetic sense coil probe time series shows the frequency spectrum broaden during the perturbation pulse, indicating a turbulent cascade. Where the turbulent spectrum is broadest $(r=0$ $\mathrm{cm})$ correlates with the obervation of the only significant ion heating.

Phase angle analysis of the magnetic sense coil probe signal shows an Alfvén wavelength an order of magnitude smaller than that predicted by 
linear theory. When the local gradient scale length and densities are included, the observed wavelength is consistent with theoretical predictions, indicating that the launched perturbations are Alfvén waves propagating in an argon helicon plasma. When the full dispersion relation is calculated, taking into account electron Landau damping, electron-ion collisions, and finite frequency effects, the observed wavelength is in even better agreement with the theoretical predictions. Time-resolved Langmuir probe measurements show an increase in electron temperature due to wave-particle interactions throughout the plasma, not just due to Ohmic heating in the driven current channel. This is an important development, since previous laboratory Alfvén waves studies have only observed Ohmic heating of electrons in the wave-driving current channel. ${ }^{[104]}$ The observed electron heating across the plasma column correlates with the observed metastable density depletion. The Langmuir probe measurements also hint at an increase in the electron density at the very center of the plasma column, but more observations are necessary to rule out experimental error.

The small amplitude of the perturbation signal in this work $(\sim 0.25 \%$ of the background field) could explain why ion heating was not observed more widely. Future measurements of these same experiments with a larger 
perturbation signal are necessary to better understand the dynamics of the system. The observation of plasma turbulence is an exciting development in this work. Further characterization of this turbulent behavior and measurement of the turbulent cascade should be undertaken. An array of magnetic sense coil probes, at multiple radial and axial locations taking simultaneous time-resolved measurements is a requirement for better understanding the plasma phenomena elucidated here. Additionally, performing the same magnetic sense coil, LIF, and Langmuir probe measurements for different argon neutral and ion density profiles across the plasma column will shed more light on how the wave propagation depends on the local plasma density, density gradient, and ion-neutral collision frequency. The observation of electron heating outside the perturbed current channel is a completely new observation in this work which validates the coronal heating models proposed by Matthaeus ${ }^{[1]}$ and Dmitruk. ${ }^{[2]}$ 


\section{Bibliography}

[1] W.H. Matthaeus, G.P. Zank, S. Oughton, D.J. Mullan, and P. Dmitruk. Coronal heating by magnetohydrodynamic turbulence driven by reflected low-frequency waves. Astrophysical Journal, Letters, 523(1):93 $-6,1999$.

[2] P. Dmitruk, W.H. Matthaeus, L.J. Milano, and S. Oughton. Conditions for sustainment of magnetohydrodynamic turbulence driven by Alfvén waves. volume 8, pages $2377-84,2001$.

[3] S. Houshmandyar. Laboratory Insvestigations of Alfvén Waves in a High Power Helicon Plasma with Density Gradients. PhD thesis, West Virginia University, 2010.

[4] R. Erdélyi and V. Fedun. Are there Alfvén waves in the solar atmosphere? Science, 318(5856):1572 - 4, 2007.

[5] P. Dmitruk, L.J. Milano, and W.H. Matthaeus. Wave-driven turbulent coronal heating in open field line regions: nonlinear phenomenological model. Astrophysical Journal, 548(1):482 - 91, 2001.

[6] M.A. Dodero, E. Antonucci, S. Giordano, and R. Martin. Solar wind velocity and anisotropic coronal kinetic temperature measured with the o vi doublet ratio. Solar Physics, 183(1):77 - 90, 1998.

[7] E. Antonucci, M.A. Dodero, and S. Giordano. Fast solar wind velocity in a polar coronal hole during solar minimum. Solar Physics, 197:115134, 2000.

[8] S.P. Gary and J.E. Borovsky. Alfven-cyclotron fluctuations: linear vlasov theory. Journal of Geophysical Research, 109(A6):10, 2004.

[9] S.R. Cranmer and A.A. van Ballegooijen. Proton heating in the extended solar corona resulting from kinetic alfven turbulence. AGU Fall Meeting Abstracts, page A407, December 2002.

[10] Benjamin D. G. Chandran. Weakly turbulent magnetohydrodynamic waves in compressible low- $\beta$ plasmas. Physical Review Letters, 101:235004, Dec 2008. 
[11] R. Erdélyi and I. Ballai. Heating of the solar and stellar coronae: a review. Astronomische Nachrichten, 328(8):726-733, 2007.

[12] W. Gekelman, S. Vincena, B. Van Compernolle, G.J. Morales, J.E. Maggs, P. Pribyl, and T.A. Carter. The many faces of shear alfven waves. Physics of Plasmas, 18(5):055501, 2011.

[13] W. Gekelman, M. VanZeeland, S. Vincena, and P. Pribyl. Experiments and observations on intense alfven waves in the laboratory and in space. Number 669, pages 408 - 11, 2003.

[14] S. Vincena and W. Gekelman. Visualizing shear alfven wave currents near the ion-cyclotron frequency. IEEE Transactions on Plasma Science, 33(2):552, 2005.

[15] N. Palmer, W. Gekelman, and S. Vincena. Measurement of ion motion in a shear alfven wave. Physics of Plasmas, 12(7):72102 - 1, 2005.

[16] S. Vincena, W. Gekelman, and J. Maggs. Shear Alfvén wave perpendicular propagation from the kinetic to the inertial regime. Physical Review Letters, 93(10):105003 - 1, 2004.

[17] J. Hanna and C. Watts. Alfvén wave propagation in a helicon plasma. Physics of Plasmas, 8(9):4251 - 4251, 2001.

[18] C. Watts and J. Hanna. Alfvén wave propagation in a partially ionized plasma. Physics of Plasmas, 11(4):1358 - 65, 2004.

[19] C. Watts. Private Communication, 2006.

[20] H. Alfvén. Existence of Electromagnetic-Hydrodynamic Waves. Nature, 150:405-406, October 1942.

[21] P. M. Bellan. Fundamentals of Plasma Physics. Cambridge University Press, Cambridge, UK.

[22] T.H. Stix. Waves in Plasmas. McGraw-Hill, 1992.

[23] S. Vincena, W. Gekelman, and J. Maggs. Shear Alfvén waves in a magnetic beach and the roles of electron and ion damping. Physics of Plasmas, 8(9):3884 - 96, 2001. 
[24] B.D. Fried and S.D. Conte. The Plasma Dispersion Function. Academic, New York, 1961.

[25] R.A. Koch and W. Horton Jr. Effects of electron angle scattering in plasma waves. Physics of Fluids, 18:861, 1975.

[26] J.W. Belcher. Alfvenic wave pressures and the solar wind. Astrophysical Journal, 168(3):509 - 24, 1971.

[27] J.-S. He, C.-Y. Tu, E. Marsch, L.-J. Guo, S. Yao, and H. Tian. Upward propagating high-frequency Alfvén waves as identified from dynamic wave-like spicules observed by sot on hinode. Astronomy amp; Astrophysics, 497(2):525 - 35, 2009.

[28] N. Nishizuka, M. Shimizu, T. Nakamura, K. Otsuji, T.J. Okamoto, Y. Katsukawa, and K. Shibata. Giant chromospheric anemone jet observed with hinode and comparison with magnetohydrodynamic simulations: evidence of propagating Alfvén waves and magnetic reconnection. Astrophysical Journal, 683:83 - 6, 2008.

[29] P. Antolin, K. Shibata, T. Kudoh, D. Shiota, and D. Brooks. Predicting observational signatures of coronal heating by Alfvén waves and nanoflares. Astrophysical Journal, 688(1):669 - 82, 2008.

[30] J.V. Hollweg. Cyclotron resonance in coronal holes. 3. a fivebeam turbulence-driven model. Journal of Geophysical Research, 105(A7):15699 - 713, 2000.

[31] T.K. Suzuki and S. Inutsuka. Making the corona and the fast solar wind: a self-consistent simulation for the low-frequency alfven waves from the photosphere to 0.3 au. Astrophysical Journal, Letters, 632(1):49 - 52, 2005.

[32] S. R. Cranmer, A. A. van Ballegooijen, and R. J. Edgar. Self-consistent coronal heating and solar wind acceleration from anisotropic magnetohydrodynamic turbulence. The Astrophysical Journal Supplement Series, 171:520-551, 2007.

[33] C.-Y. Tu and E. Marsch. Wave dissipation by ion cyclotron resonance in the solar corona. Astronomy and Astrophysics, 368(3):1071 - 1076, 2001. 
[34] J.V. Hollweg and P.A. Isenberg. Generation of the fast solar wind: A review with emphasis on the resonant cyclotron interaction. Journal of Geophysical Research (Space Physics), 107:1147, 2002.

[35] Martin I. Panevsky and Roger D. Bengtson. Characterization of the resonant electromagnetic mode in helicon discharges. Physics of Plasmas, 11:4196 - 4205, 2004.

[36] K. Itoh, S.-I. Itoh, P.H. Diamond, T.S. Hahm, A. Fujisawa, G.R. Tynan, M. Yagi, and Y. Nagashima. Physics of zonal flows. volume 13, 2006.

[37] G.W. Gibson Jr. and D.J. Hemker. Integrated stack etching using a helicon plasma source. Semiconductor International, 19:193 - 4, 1996.

[38] S. Kimura and H. Ikoma. Fowler-nordheim current injection and write/erase characteristics of metal-oxide-nitride-oxide-si structure grown with helicon-wave excited plasma processing. Journal of Applied Physics, 85:551 - 7, 1999.

[39] J.P. Squire. Progress in experimental research of the VASIMR engine. Fusion Sci. Technol, 43:111-117, January 2003.

[40] R. W. Boswell, O. Sutherland, C. Charles, J. P. Squire, F. R. Chang Diaz, T. W. Glover, V. T. Jacobson, D. G. Chavers, R. D. Bengtson, E. A. Bering III, R. H. Goulding, and M. Light. Experimental evidence of parametric decay processes in the variable specific impulse magnetoplasma rocket (vasimr) helicon plasma source. Physics of Plasmas, 11:5125 - 5129, 2004.

[41] Xuan Sun, Costel Biloiu, Robert Hardin, and Earl E. Scime. Parallel velocity and temperature of argon ions in an expanding, helicon source driven plasma. Plasma Sources Science and Technology, 13:359 - 370, 2004.

[42] R. Winglee, T. Ziemba, L. Giersch, J. Prager, J. Carscadden, and B.R. Roberson. Simulation and laboratory validation of magnetic nozzle effects for the high power helicon thruster. Physics of Plasmas, 14, 2007. 
[43] E.E. Scime, P.A. Keiter, M.M. Balkey, R.F. Bolvin, J.L. Kline, M. Blackburn, and S.P. Gary. Ion temperature anisotropy limitation in high beta plasmas. volume 7, pages $2157-65,2000$.

[44] J. Hanna and C. Watts. Alfven wave propagation in a helicon plasma. Physics of Plasmas, 8:4251 - 4, 2001.

[45] L Del Zanna and M Velli. Coronal heating through alfvén waves. Advances in Space Research, 30:471-480, 2002.

[46] C. Watts and J. Hanna. Alfven wave propagation in a partially ionized plasma. Physics of Plasmas, 11:1358 - 65, 2004.

[47] S. Houshmandyar and E. E. Scime. Ducted kinetic Alfvén waves in plasma with steep density gradients. Physics of Plasmas, 18:112111, November 2011.

[48] F.F. Chen. Physics of helicon discharges. volume 3, pages 1783 - 93, 1996.

[49] Paul A. Keiter, Earl E. Scime, and Matthew M. Balkey. Frequency dependent effects in helicon plasmas. Physics of Plasmas, 4(7):2741 $2747,1997$.

[50] A.R. Ellingboe and R.W. Boswell. Capacitive, inductive and heliconwave modes of operation of a helicon plasma source. Physics of Plasmas, 3(7):2797 - 804, 1996.

[51] R.W. Boswell and F.F. Chen. Helicons-the early years. IEEE Transactions on Plasma Science, 25(6):1229 - 44, 1997.

[52] F.F. Chen and R.W. Boswell. Helicons-the past decade. IEEE Transactions on Plasma Science, 25(6):1245 - 57, 1997.

[53] R.L. Kinder and M.J. Kushner. Wave propagation and power deposition in magnetically enhanced inductively coupled and helicon plasma sources. Journal of Vacuum Science amp; Technology A (Vacuum, Surfaces, and Films), 19(1):76 - 86, 2001.

[54] G.G. Borg, J. Bright, and I.V. Kamenski. Collisionless energy coupling to high-velocity electrons in the near field of an antenna: neutral gas 
ionization by helicon waves. Plasma Physics and Controlled Fusion, 40(6):987 - 1000, 1998.

[55] A.W. Degeling and R.W. Boswell. Modeling ionization by helicon waves. Physics of Plasmas, 4(7):2748 - 55, 1997.

[56] Y. Mouzouris and J.E. Scharer. Wave propagation and absorption simulations for helicon sources. Physics of Plasmas, 5(12):4253 - 61, 1998.

[57] F.F. Chen and D.D. Blackwell. Upper limit to landau damping in helicon discharges. Physical Review Letters, 82(13):2677 - 80, 1999.

[58] Suwon Cho and Jong-Gu Kwak. Effects of the density profile on the power absorption and the equilibrium density in helicon plasmas. Physics of Plasmas, 4(11):4167 - 4172, 1997.

[59] R.T.S. Chen and N. Hershkowitz. Multiple electron beams generated by a helicon plasma discharge. Physical Review Letters, 80(21):4677 80, 1998.

[60] M.M. Balkey. Optimization of a Helicon Plasma Source for Maximum Density with Minimal Ion Heating. PhD thesis, West Virginia University, 2000 .

[61] I. Bilou. Laser Induced Fluorescence studies of ion acceleration in single and multiple species expanding helicon plasma. $\mathrm{PhD}$ thesis, West Virginia University, 2009.

[62] J. Kline. Resonant ion heating in helicon plasma. Master's thesis, West Virginia University, 1998.

[63] J.M. Beall, Y.C. Kim, and E.J. Powers. Estimation of wavenumber and frequency spectra using fixed probe pairs. Journal of Applied Physics, 53(6):3933 - 3940, 1982.

[64] K.-M. Lau and Hengyi Weng. Climate signal detection using wavelet transform: how to make a time series sing. Bulletin - American Meteorological Society, 76(12):2391 - 2402, 1995. 
[65] J. Carr Jr. Laser Induced Fluorescence Studies of Electrostatic Double Layers in an Expanding Helicon Plasma. PhD thesis, West Virginia University, 2013.

[66] C.K. Chui. An Introduction to Wavelets. Academic Press, San Diego, 1992.

[67] R.X. Gao and R. Yan. Wavelets: Theory and Applications for Manufacturing. Springer Science+Business Media, LLC, New York, 2011.

[68] R.N. Bracwell. The Fourier Transform and Its Applications. McGrawHill Book Company, 1978.

[69] J.S. Walker. A Primer on WAVELETS and Their Scientific Applications. Chapman Hall/CRC Taylor Francis Group, LLC, Boca Raton, 2008.

[70] M.T. Heideman, D.H. Johnson, and C.S. Burrus. Gauss and the history of the fast fourier transform. IEEE ASSP Magazine, 1(4):14-21, 1984.

[71] B.B. Hubbard. The World According to Wavelets: The Story of a Mathematical Technique in the Making. A K Peters, Natick, 1998.

[72] C. Torrence and G.P. Compo. Bulletin of the American Meteorological Society, 79:61, 1998.

[73] C. Torrence and P.J. Webster. Journal of Climate, 12:2679, 1999.

[74] B. Ph. van Milligen, C. Hidalgo, and E. Sánchez. Nonlinear phenomena and intermittency in plasma turbulence. Physical Review Letters, 74:395-398, Jan 1995.

[75] F. Brochard, T. Windisch, O. Grulke, and T. Klinger. Experimental evidence of mode coupling in drift wave intermittent turbulence using a wave number bicoherence analysis. Physics of Plasmas, 13(12), 2006.

[76] S. Oldenburger, S. Inagaki, T. Kobayashi, H. Arakawa, N. Ohyama, K. Kawashima, Y. Tobimatsu, A. Fujisawa, K. Itoh, and S.-I. Itoh. Dynamics of particle flux in a cylindrical magnetized plasma. Plasma Physics and Controlled Fusion, 54(5), 2012. 
[77] E.E. Scime, J. Carr Jr., M. Galante, R.M. Magee, and R. Hardin. Ion heating and short wavelength fluctuations in a helicon plasma source. Physics of Plasmas, 20(3), 2013.

[78] M. Farge. Wavelet transforms and their applications to turbulence. Annual Review of Fluid Mechanics, 24(1):395-458, 1992.

[79] Y. Meyer. In Wavelets: Algorithms 6 Applications. Society for Industrial and Applied Mathematics, Philadelphia.

[80] I. Daubechies. Ten Lectures on Wavelets. Society for Industrial and Applied Mathematics, Philadelphia, 1992.

[81] Xuan Sun, C. Biloiu, and E. Scime. Observation of resistive drift Alfvén waves in a helicon plasma. Physics of Plasmas, page 102105, 2005.

[82] S. Chakraborty Thakur, D. McCarren, T. Lee, N. Fedorczak, P. Manz, E.E. Scime, G.R. Tynan, and M. Xu. Laser induced fluorescence measurements of ion velocity and temperature of drift turbulence driven sheared plasma flow in a linear helicon plasma device. Physics of Plasmas, 19(8):082102, 2012.

[83] E. Scime, R. Hardin, C. Biloiu, A.M. Keesee, and X. Sun. Flow, flow shear, and related profiles in helicon plasmas. Physics of Plasmas, 14(4):43505, 2007.

[84] J.T. Yardley and C. Bradley Moore. Laser-excited vibrational fluorescence and energy transfer in methane. Journal of Chemical Physics, 45(3):1066 - 1067, 1966.

[85] R.A. Stern and III Johnson, J.A. Plasma ion diagnostics using resonant fluorescence. Physical Review Letters, 34(25):1548 - 51, 1975.

[86] H.C. Meng and H.-J. Kunze. Investigation of the diffusion of impurity atoms in plasmas by laser fluorescence. Physics of Fluids, 22(6):1082 $-8,1979$.

[87] David N. Hill, Steven Fornaca, and Michael G. Wickham. Single frequency scanning laser as a plasma diagnostic. Review of Scientific Instruments, 54(3):309 - 314, 1983. 
[88] A.M. Keesee. Neutral Density Profiles in Argon Helicon Plasmas. PhD thesis, West Virginia University, 2006.

[89] R.F. Boivin. Study of different line broadening mechanisms for the laser induced fluorescence diagnostics of the helix and leia plasmas. Technical Report WVU-Plasma Group Internal Reports- PL-039, West Virginia University, 1998.

[90] X. Sun. A Study of Ion Acceleration, Asymmetric Optical Pumping and Low Frequency Waves in Two Expanding Helicon Plasmas. PhD thesis, West Virginia University, 2005.

[91] R.F. Boivin. Zeeman splitting for lif transitions and de-convolution technique to extract ion temperatures. Technical Report WVU-Plasma Group Internal Reports- PL-050, West Virginia University, 2002.

[92] H. M. Mott-Smith and Irving Langmuir. The theory of collectors in gaseous discharges. Phys. Rev., 28:727-763, Oct 1926.

[93] I. H. Hutchinson. Principles of Plasma Diagnostics. Cambridge University Press, 1987.

[94] I. H. Hershkowitz. How langmuir probes work. In O.Aucillo and. D.L. Flamm, editor, Plasma Diagnostics, Discharge Parameters and Chemistry. Academic Press, 1998.

[95] F. F. Chen. In R. H. Huddlestone and S. L. Leonard, editors, Plasma Diagnostic Techniques. Academic Press, New York, 1965.

[96] L. Schott. In W. Lochte-Holtgreven, editor, Plasma Diagnostics. John Wiley, New York, 1968.

[97] V.I. Demidov, S.V. Ratynskaia, and K. Rypdal. Electric probes for plasmas: The link between theory and instrument. Review of Scientific Instruments, 73(10):3409 - 3409, 2002.

[98] T. E. Sheridan and J. Goree. Langmuir-probe characteristic in the presence of drifting electrons. Phys. Rev. E, 50:2991-2996, Oct 1994.

[99] N.A. Krall and A.W. Trivelpiece. Principles of plasma physics. McGraw-Hill, 1973. 
[100] P.A. Keiter, E.E. Scime, M.M. Balkey, R. Boivin, J.L. Kline, and S.P. Gary. Beta-dependent upper bound on ion temperature anisotropy in a laboratory plasma. Physics of Plasmas, 7(3):779 - 83, 2000.

[101] J.C. Sprott. Electrostatic probe techniques. Technical Report PLP 88, University of Wisconsin, Madison, 1966.

[102] I.D. Sudit and F.F. Chen. Rf compensated probes for high-density discharges. Plasma Sources Science and Technology, 3(2):162, 1994.

[103] R.A. Hardin. Measurement of Short-Wavelength Electrostatic Fluctuations in a Helicon Plasma Source. PhD thesis, West Virginia University, 2008.

[104] W. Gekelman. Email Correspondence, 2014. 


\section{Appendix A: MATLAB Code for Analyzing Time-Resolved Langmuir Probe Data}

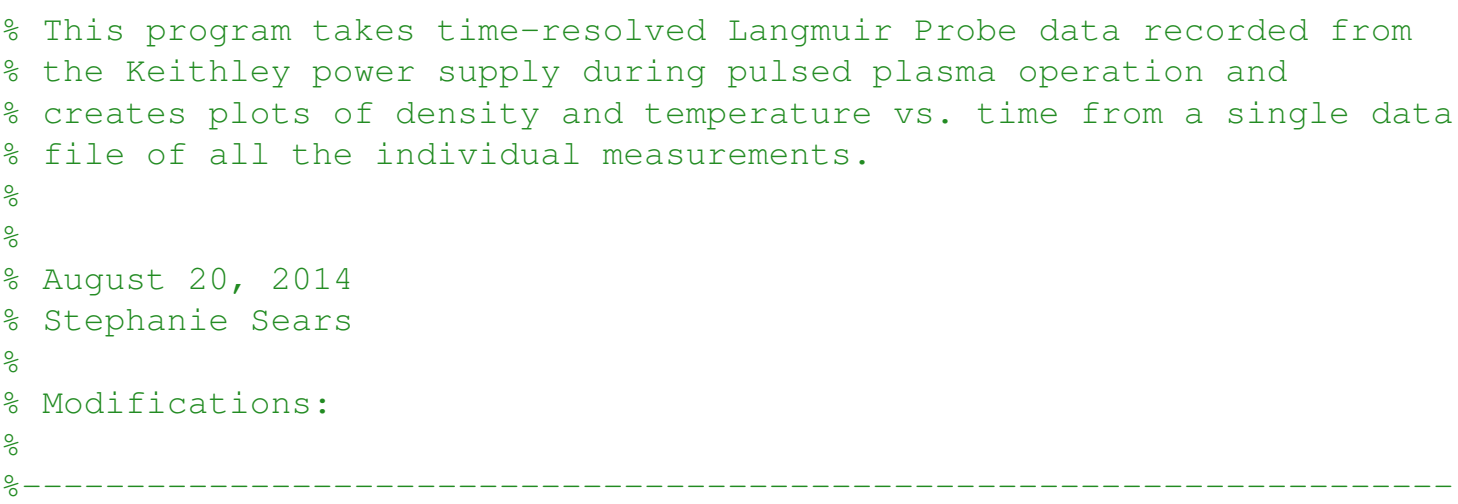




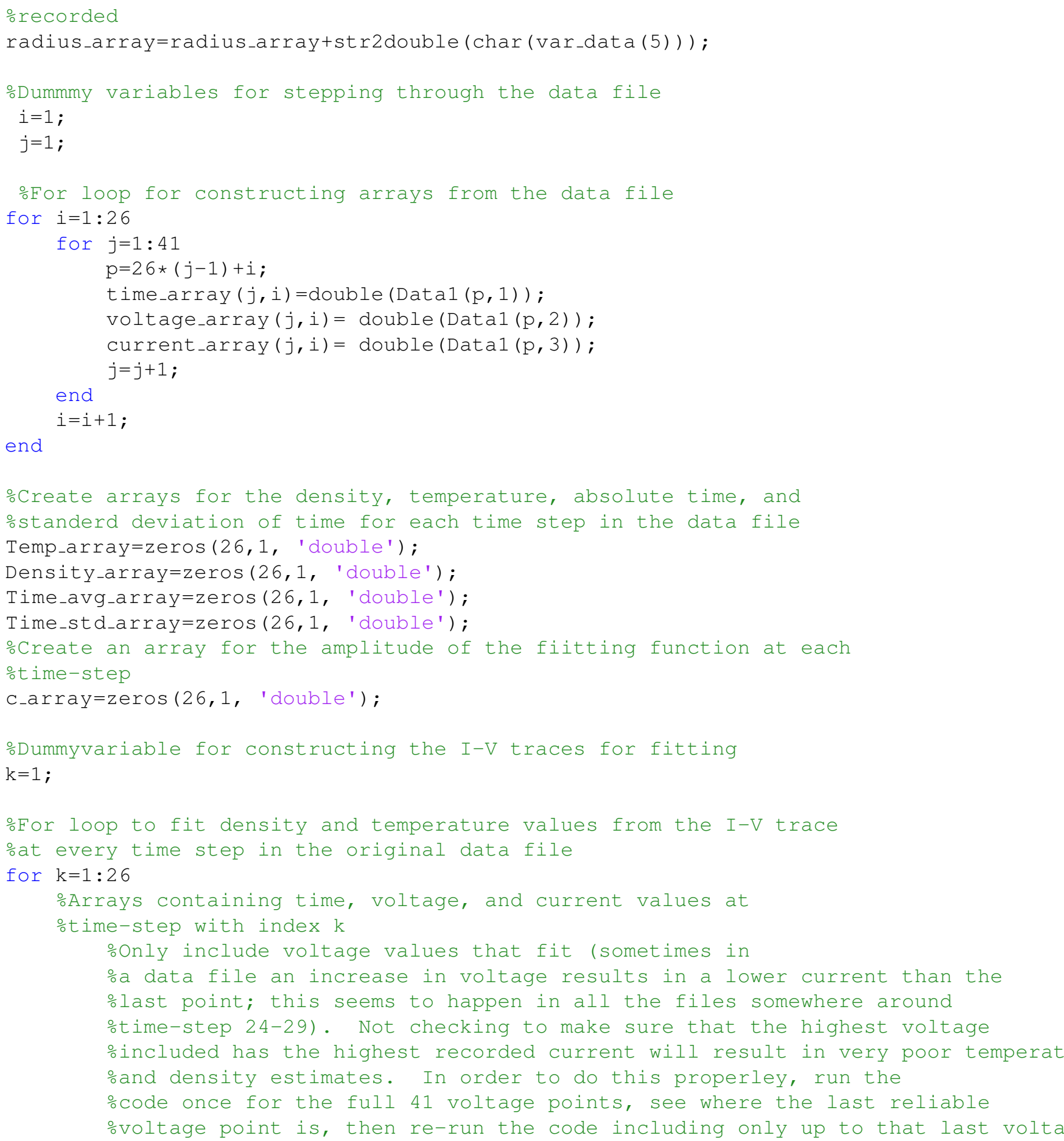




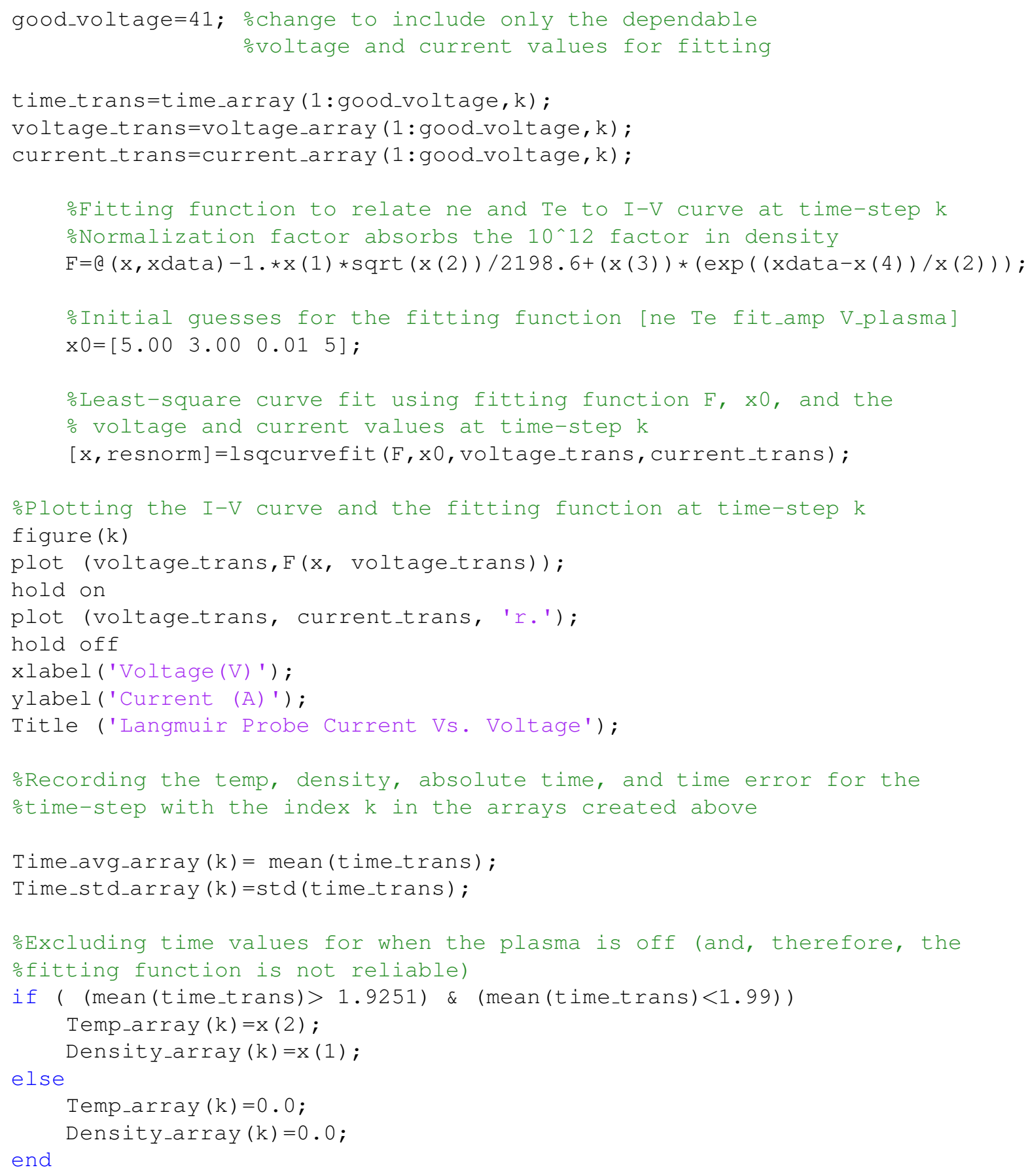




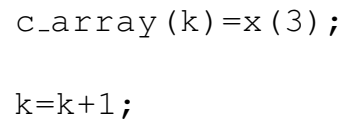




\section{Appendix B: MATLAB Code for Wavelet Analysis}

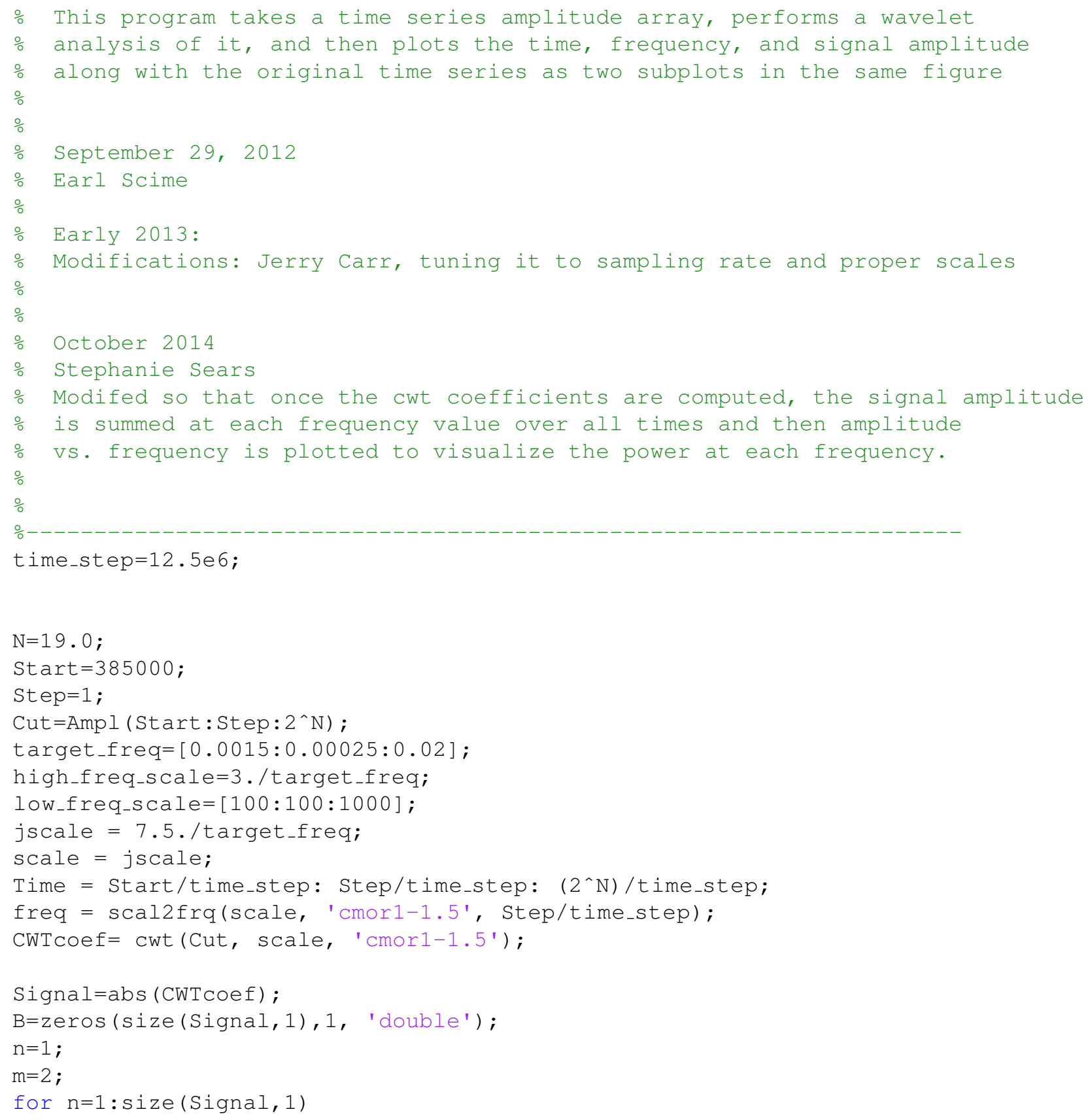




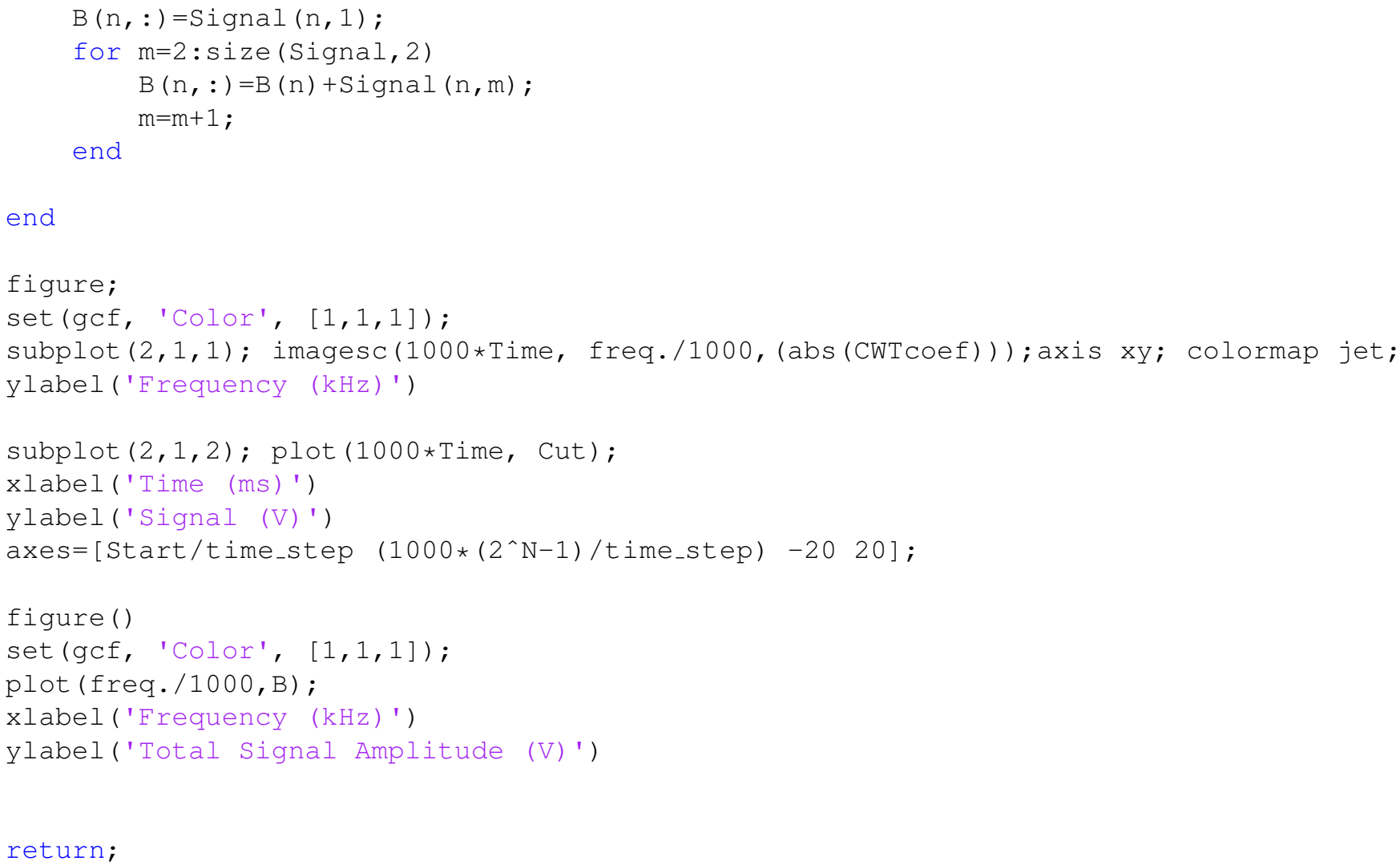

